\title{
Radioactive nuclei in the early Solar System: analysis of the 15 isotopes produced by core-collapse supernovae
}

\author{
Thomas V. Lawson ${ }^{1,2,3,4,5}$, Marco Pignatari ${ }^{1,2,3,4,5}$, Richard J. Stancliffe , $^{1,3,5,6}$, \\ Jacqueline den Hartogh ${ }^{2,3}$, Sam Jones ${ }^{3,7}$, Chris L. Fryer ${ }^{3,7}$, Brad K. Gibson ${ }^{1,4,5}$, \\ Maria Lugaro $2,8,9$ \\ ${ }^{1}$ E. A. Milne Centre for Astrophysics, Department of Physics and Mathematics, University of Hull, HU6 7RX, United Kingdom
}

${ }^{2}$ Konkoly Observatory, Research Centre for Astronomy and Earth Sciences, Eötvös Loránd Research Network (ELKH), Konkoly Thege Miklós út 15-17, H-1121 Budapest, Hungary

${ }^{3}$ NuGrid Collaboration, http: // nugridstars. org

${ }^{4}$ Joint Institute for Nuclear Astrophysics - Center for the Evolution of the Elements

${ }^{5}$ BridGCE: Bridging Disciplines of Galactic Chemical Evolution

${ }^{6}$ H. H. Wills Physics Laboratory, Tyndall Avenue, Bristol BS8 1TL, UK

${ }^{7}$ X Computational Physics (XCP) Division, Los Alamos National Laboratory, Los Alamos, NM 87545, USA

${ }^{8}$ School of Physics and Astronomy, Monash University, VIC 3800, Australia

${ }^{9}$ ELTE Eötvös Loránd University, Institute of Physics, Budapest 1117, Pázmány Péter sétány 1/A, Hungary

Accepted XXX. Received YYY; in original form ZZZ

\begin{abstract}
Short-lived radioactive isotopes (SLRs) with half-lives between 0.1 to 100 Myr can be used to probe the origin of the Solar System. In this work, we examine the core-collapse supernovae production of the 15 SLRs produced: ${ }^{26} \mathrm{Al},{ }^{36} \mathrm{Cl},{ }^{41} \mathrm{Ca},{ }^{53} \mathrm{Mn},{ }^{60} \mathrm{Fe},{ }^{92} \mathrm{Nb},{ }^{97} \mathrm{Tc},{ }^{98} \mathrm{Tc},{ }^{107} \mathrm{Pd},{ }^{126} \mathrm{Sn},{ }^{129} \mathrm{I},{ }^{135} \mathrm{Cs}$, ${ }^{146} \mathrm{Sm},{ }^{182} \mathrm{Hf}$, and ${ }^{205} \mathrm{~Pb}$. We probe the impact of the uncertainties of the core-collapse explosion mechanism by examining a collection of 62 core-collapse models with initial masses of 15,20 , and 25 $\mathrm{M}_{\odot}$, explosion energies between $3.4 \times 10^{50}$ and $1.8 \times 10^{52}$ ergs and compact remnant masses between $1.5 \mathrm{M}_{\odot}$ and $4.89 \mathrm{M}_{\odot}$. We identify the impact of both explosion energy and remnant mass on the final yields of the SLRs. Isotopes produced within the innermost regions of the star, such as ${ }^{92} \mathrm{Nb}$ and ${ }^{97} \mathrm{Tc}$, are the most affected by the remnant mass, ${ }^{92} \mathrm{Nb}$ varying by five orders of magnitude. Isotopes synthesised primarily in explosive C-burning and explosive He-burning, such as ${ }^{60} \mathrm{Fe}$, are most affected by explosion energies. ${ }^{60} \mathrm{Fe}$ increases by two orders of magnitude from the lowest to the highest explosion energy in the $15 \mathrm{M}_{\odot}$ model. The final yield of each examined SLR is used to compare to literature models.
\end{abstract}

Key words: Stellar types: Massive stars - Supernovae - Nucleosynthesis

\section{INTRODUCTION}

Radioactive isotopes are useful tools to determine the age of objects. On Earth, ${ }^{14} \mathrm{C}$ (with a half-life, $\mathrm{t}_{\frac{1}{2}}$, of $5730 \mathrm{yr}$ ) is used to measure timescales relative to human history. In the Universe, long lived isotopes $\left(\mathrm{t}_{\frac{1}{2}} \approx \mathrm{Gyr}\right)$ such as ${ }^{232} \mathrm{Th}$ or ${ }^{238} \mathrm{U}$ are used to measure cosmological timescales like the age of the Galaxy (Dauphas 2005). The process of the formation of the Sun, starting from the formation of its molecular cloud, lasts between 15 years (Hartmann et al. 2001; Murray 2011), therefore, in order to probe this time period we examine isotopes that have comparable decay times. We use radioactive isotopes with half-lives between 0.1 and $100 \mathrm{Myr}$ (short-lived radionuclides: SLRs, henceforth) as chronometers for understanding the birth of the Sun. The abundances of SLRs in the early Solar System (ESS) are derived from meteoritic analysis.

SLR signatures can be used alongside expected contributions from galactic chemical evolution (GCE) to identify the time elapsed from production to being incorporated into the ESS; this is referred to as the so-called 'isolation time' (Côté et al. 2019a,b; Yagüe López et al. 2021). SLRs with the shortest half-lives completely decay over long timescales ( $100 \mathrm{Myr})$. Therefore, to explain the abundance of the shortest lived isotopes, such as ${ }^{26} \mathrm{Al},{ }^{36} \mathrm{Cl}$ and ${ }^{41} \mathrm{Ca}$ (half lives of these isotopes can be found in Table 1), we require additional, local sources that are close in both time and space (Huss et al. 2009). These local sources may have polluted either the molecular cloud or the proto-solar disk (see the review by Lugaro et al. 2018). 


\begin{tabular}{cccc}
\hline SLR & Daughter & Reference & $\mathrm{T}_{1 / 2}(\mathrm{Myr})$ \\
\hline${ }^{26} \mathrm{Al}$ & ${ }^{26} \mathrm{Mg}$ & ${ }^{27} \mathrm{Al}$ & 0.72 \\
${ }^{36} \mathrm{Cl}$ & ${ }^{36} \mathrm{~S}$ & ${ }^{35} \mathrm{Cl}$ & 0.30 \\
${ }^{41} \mathrm{Ca}$ & ${ }^{41} \mathrm{~K}$ & ${ }^{40} \mathrm{Ca}$ & 0.099 \\
${ }^{53} \mathrm{Mn}$ & ${ }^{53} \mathrm{Cr}$ & ${ }^{55} \mathrm{Mn}$ & 3.7 \\
${ }^{60} \mathrm{Fe}$ & ${ }^{60} \mathrm{Ni}$ & ${ }^{56} \mathrm{Fe}$ & 2.6 \\
${ }^{92} \mathrm{Nb}$ & ${ }^{92} \mathrm{Zr}$ & ${ }^{92} \mathrm{Mo}$ & 34 \\
${ }^{97} \mathrm{Tc}$ & ${ }^{97} \mathrm{Mo}$ & ${ }^{98} \mathrm{Ru}$ & 4.2 \\
${ }^{98} \mathrm{Tc}$ & ${ }^{98} \mathrm{Ru}$ & ${ }^{98} \mathrm{Ru}$ & 4.2 \\
${ }^{107} \mathrm{Pd}$ & ${ }^{107} \mathrm{Ag}$ & ${ }^{108} \mathrm{Pd}$ & 6.5 \\
${ }^{126} \mathrm{Sn}$ & ${ }^{126} \mathrm{Te}$ & ${ }^{124} \mathrm{Sn}$ & 0.23 \\
${ }^{129} \mathrm{I}$ & ${ }^{129} \mathrm{Xe}$ & ${ }^{127} \mathrm{I}$ & 15 \\
${ }^{135} \mathrm{Cs}$ & ${ }^{135} \mathrm{Ba}$ & ${ }^{133} \mathrm{Cs}$ & 2.3 \\
${ }^{146} \mathrm{Sm}$ & ${ }^{142} \mathrm{Nd}$ & ${ }^{144} \mathrm{Sm}$ & 68 \\
${ }^{182} \mathrm{Hf}$ & ${ }^{182} \mathrm{~W}$ & ${ }^{180} \mathrm{Hf}$ & 8.9 \\
${ }^{205} \mathrm{~Pb}$ & ${ }^{205} \mathrm{Tl}$ & ${ }^{204} \mathrm{~Pb}$ & 17
\end{tabular}

Table 1. SLRs considered in this work. SLRs are listed with daughter isotopes, reference isotopes and Half-lives $\left(\mathrm{T}_{1 / 2}\right)$. Data gathered from Lugaro et al. (2018).

Huss et al. (2009) presented a comparison of the potential sources of SLRs into the ESS, concluding that intermediate-mass asymptotic giant branch (AGB) stars and massive stars between $20-60 \mathrm{M}_{\odot}$ provide the most feasible sources. The AGB scenario has been considered (see Wasserburg et al. 2017) and compared to massive stars (Vescovi et al. 2018). The latter find that the contamination of a solar nebula by a single core-collapse supernova (CCSN) event should also pollute stable isotopes to excess. Meyer \& Clayton (2000) examines the different SLR yields in the Solar System and the values expected from GCE approaches, including a comparison to the massive star models of Meyer et al. (1995).

A popular ESS pollution scenario is a single CCSN event, which polluted the material that formed the Solar System. A supernova could also explain the formation of the Solar System itself, by shocking the gas cloud that would go on to form the Solar System (as first proposed by Cameron \& Truran (1977)). During this shock we can expect the ejecta of this supernova event to enrich the cloud (Gritschneder et al. 2012) or the disk (Portegies Zwart et al. 2018). Boss \& Keiser (2014) and Boss (2017) show that a collapse caused by a supernova can explain both ${ }^{60} \mathrm{Fe}$ and ${ }^{26} \mathrm{Al}$, but a problem with this is that it can lead to an overenhancement of ${ }^{53} \mathrm{Mn}$ up to three orders of magnitude. The ${ }^{53} \mathrm{Mn}$ overproduction can be solved by allowing a larger fallback during the CCSN (see Takigawa et al. 2008), where fallback is defined as the inner region of the star that collapses onto the neutron star or black hole remnant. A chemo hydro-dynamical simulation of the entire Milky Way Galaxy by Fujimoto et al. (2018) examined the impact of individual CCSNe to SLR abundances, finding that pollution by CCSNe provides significant amounts of ${ }^{26} \mathrm{Al}$ and ${ }^{60} \mathrm{Fe}$. However, this work considered only two SLRs.

Previous works that examine CCSN scenario yields have only examined a selection of SLRs. Timmes et al. (1995) examined the GCE component of ${ }^{26} \mathrm{Al}$ and ${ }^{60} \mathrm{Fe}$ in the Milky Way using the CCSN yields from Woosley \& Weaver (1995). Meyer et al. (1995) examined the yield for the ejecta of a $25 \mathrm{M}_{\odot}$ star, including yields for ${ }^{26} \mathrm{Al},{ }^{36} \mathrm{Cl},{ }^{41} \mathrm{Ca}$, ${ }^{53} \mathrm{Mn}$ and ${ }^{60} \mathrm{Fe}$. In this work we continue the work done by Jones et al. (2019) and Andrews et al. (2020) to study the production of radioactive isotopes in CCSNe. Our nucleosynthesis simulations cover the full range of explosion parameters as described in Fryer et al. (2018). We aim to study the production of all SLRs presented in Table 1 in CCSNe (Lugaro et al. 2018). In particular, we discuss the impact of the explosion parameters affecting the production of the SLRs in CCSN ejecta.

The paper is organized as follows. In Section 2 we describe the stellar models and the nucleosynthesis code. In Section 3, we present the results for the production and abundances of the SLRs. The discussion about the impact of model parameters on the SLR abundances and the comparison with other models are given in Section 4. In Section 5, we summarize our results.

\section{SIMULATIONS}

We first describe the CCSN models used in this work, in particular which parameters are altered, as well as the method of pre-explosive and explosive nucleosynthesis. We then give a brief explanation of the nucleosynthesis that has taken place, calling into focus the key explosive nucleosynthesis sites.

\subsection{Methods and Models}

We use the CCSN models calculated by Fryer et al. (2018) on the basis of three progenitor models with initial masses of 15,20 and $25 \mathrm{M}_{\odot}$ computed by Heger \& Woosley (2010) with the KEPLER code (Weaver et al. 1978; Woosley \& Weaver 1995) and with initial metallicity (Z) of 0.02 (Grevesse \& Noels 1993). The explosions of each progenitor were calculated using a 1-dimensional Lagrangian hydrodynamic code, to mimic a 3-dimensional convective engine (Fryer et al. 1999; Herant et al. 1994) by injecting energy above the proto-neutron star with three free parameters: the mass at which the energy is injected, the length of time of the energy injection, and the total energy injected. Fryer et al. (2018) use these three parameters to study the uncertainty of the explosive engine, and to determine the uncertainty on the remnant mass and isotopic yield of each explosion model. The compact remnant left after core-collapse and fallback will form either a neutron star or black hole, depending on the final mass of the compact object. The heaviest neutron star known is MSP J0740+6620, with a mass of $2.14_{-0.20}^{+0.18} \mathrm{M}_{\odot}$ (Cromartie et al. 2020). In general, we may assume that remnants with masses lower than MSP J0740+6620 form neutron stars, while heavier remnants will form black holes. Theoretical simulations of the critical mass boundary dividing neutron star and black hole formation are affected from several uncertainties, among others from the Equation of State to use for the neutron star interior (e.g., Özel \& Freire 2016). Therefore, at present the observations of the most massive neutron stars provides the best available constraint of such a mass boundary.

From this parameter study we examine 17, 23 and 22 models of progenitor mass 15,20 , and $25 \mathrm{M}_{\odot}$, respectively. The ranges of the values of the explosion energy and remnant mass are listed in Table 2, with each model's specific resultant explosion energy and remnant mass of the compact 


\begin{tabular}{cccc}
\hline $\begin{array}{c}\mathrm{M}_{\text {prog }} \\
\left(\mathrm{M}_{\odot}\right)\end{array}$ & $\begin{array}{c}\text { Model } \\
\text { count }\end{array}$ & $\begin{array}{c}\mathrm{E}_{\text {exp }} \\
\left(\times 10^{51} \text { ergs }\right)\end{array}$ & $\begin{array}{c}\mathrm{M}_{\text {rem }} \\
\left(\mathrm{M}_{\odot}\right)\end{array}$ \\
\hline 15 & 17 & $0.34-10.7$ & $1.50-1.94$ \\
20 & 23 & $0.53-8.86$ & $1.74-3.40$ \\
25 & 22 & $0.89-9.73$ & $1.83-4.89$
\end{tabular}

Table 2. Parameters of CCSN explosion models: $M_{\text {prog }}$ is the zero age main sequence mass, $E_{\text {exp }}$ is the explosion energy, and $M_{r e m}$ is the final mass of the compact object left from the SN explosion (a Neutron Star or a Black Hole).

object listed in Tables A1-A5 (Fryer et al. 2018). As a result of the parameter space explored by Fryer et al. (2018), a large range of explosion energies and remnant masses are given for each stellar progenitor. We note that when describing a model we use the term "mass cut" to define a point in mass below which material falls back back onto the compact remnant. Mass cut is commonly determined by placing it where the ejected yield of ${ }^{56} \mathrm{Ni}$ is in agreement with CCSN light curves, however in the models examined here mass cut is based on the physical properties of the explosion (See Section 2 of Fryer et al. 2018, for a description).

We perform the nucleosynthesis calculations using $\mathrm{Nu}-$ Grid post-processing tools, described in detail in Jones et al. (2019) and Andrews et al. (2020). For the massive star progenitors we use the Multi-zone Post-Processing Network Parallel (MPPNP) code (Pignatari et al. 2016b; Ritter et al. 2018). This code calculates the nuclear reactions at each time step then performs mixing across the stellar model, separate to calculating the impact of nuclear reactions. The reaction network consists of roughly 1100 isotopes and 14000 reactions. For the core-collapse simulations we used the Tracer particle Post-Processing Network - Parallel (TPPNP) (Jones et al. 2019). TPPNP functions by considering single tracer particles anchored to a mass coordinate, post-processing with no mixing between particles. We use a network of up to 5200 isotopes and 67000 reactions for CCSN nucleosynthesis (for a full description of post-processing techniques see Jones et al. 2019). This network is larger than that in the MPPNP calculations because explosive nucleosynthesis experiences higher temperatures, as well as significantly higher neutron and proton densities. Therefore, more isotopes and reactions are needed. We note that our ${ }^{26} \mathrm{Al}$ yields are higher than those reported in Jones et al (2019) and Andrews et al. (2020). In those calculations, the pre-explosive abundance of only the ${ }^{26} \mathrm{Al}$ isomeric state was inadvertently used as initial abundance of ${ }^{26} \mathrm{Al}$ during the supernova nucleosynthesis calculations. In this work this issue with the treatment of the initial abundance of the isomeric and ground states of ${ }^{26} \mathrm{Al}$ is corrected. As a result, higher final ${ }^{26} \mathrm{Al}$ yields are obtained since also the pre-explosive contribution is consistently taken into account when the final integrated yields are calculated from the SN ejecta.

\subsection{General nucleosynthesis results}

We examine the general nucleosynthesis in the 62 CCSN models described in the previous subsection ${ }^{1}$ (Fryer et al. 2018; Andrews et al. 2020). The final yields of SLRs and their reference isotopes, explosive energy and remnant masses are reported in Table A1. Here we focus on three selected models to discuss the nucleosynthesis both preexplosion and post-explosion. Figures 1, 2, and 3 show selected abundance profiles for pre- and post-CCSN of selected models. These models are selected due to their low mass cut values, allowing the largest range of explosive nucleosynthesis to be observed.

The shaded areas of Figs. 1, 2 and 3 show the location of the pre-CCSN burning ashes, as labeled at the top of the figure. These regions can be identified by the significant reduction of the relevant isotope, due to the previous burning phases. Using the top panel of Fig. 1 as an illustrative example, at mass coordinate $3.21 \mathrm{M}_{\odot}$ a sharp increase in both ${ }^{12} \mathrm{C}$ and ${ }^{16} \mathrm{O}$ represent the interface between the He-burning ashes and the H-burning ashes. The He-burning ashes extend to $2.57 \mathrm{M}_{\odot}$, and is split at $2.87 \mathrm{M}_{\odot}$ into He and $\mathrm{He}_{\text {inc }}$, which are the ashes of complete and incomplete He-burning, respectively. During He-burning the triple- $\alpha$ reaction has produced ${ }^{12} \mathrm{C}$, with a further $\alpha$ capture generating ${ }^{16} \mathrm{O}$. The ashes of C-burning are identifiable by the significant reduction in the abundance of ${ }^{12} \mathrm{C}$ between $1.78 \mathrm{M}_{\odot}$ and $2.57 \mathrm{M}_{\odot}$. During $\mathrm{C}$-burning, ${ }^{12} \mathrm{C}$ is consumed via the $\mathrm{C}$ fusion main channels ${ }^{12} \mathrm{C}\left({ }^{12} \mathrm{C}, \alpha\right){ }^{20} \mathrm{Ne}$ and ${ }^{12} \mathrm{C}\left({ }^{12} \mathrm{C}, \mathrm{p}\right){ }^{23} \mathrm{Na}$.

At $1.77 \mathrm{M}_{\odot}$ the sharp drop in ${ }^{20} \mathrm{Ne}$ and the increase of ${ }^{16} \mathrm{O}$ (created via the ${ }^{20} \mathrm{Ne}(\gamma, \alpha){ }^{16} \mathrm{O}$ reaction) represent the interface between the C-burning and Ne-burning ashes. At $1.71 \mathrm{M}_{\odot}$ the sharp drop in ${ }^{16} \mathrm{O}$ and the sharp increase of ${ }^{28} \mathrm{Si}$ define the beginning of the O-burning ashes, whose burning is dominated by the ${ }^{16} \mathrm{O}\left({ }^{16} \mathrm{O}, \alpha\right){ }^{28} \mathrm{Si}$ reaction. The Si-burning ashes are located below $1.59 \mathrm{M}_{\odot}$, where there is a sharp drop in ${ }^{28} \mathrm{Si}$. Silicon burning is characterised by hundreds of reactions, where a significant fraction of forward and backward nuclear reactions balance (Woosley et al. 1973; Chieffi et al. 1998). The main product of Si-burning is ${ }^{56} \mathrm{Fe}$.

The impact of the shock wave on the final yields of a CCSN model can be determined by examining the temperature profiles, as explosive nucleosynthesis processes have a strong temperature dependence. The mass of the remnant, and thus the location of the mass cut, is also key to determining the yields of a CCSN model, as everything above the remnant mass is ejected. In other words, if the mass cut is above a specific explosive nucleosynthesis region, then the products of that region are not ejected, therefore they are not included in the final yields.

As the explosion shock-wave passes through the preCCSN material, the temperature and densities rise dramatically (to the peak values shown in Fig. 4) and subsequently cool. These profiles determine the outcome of the explosive nucleosynthesis. During explosive nucleosynthesis (solid lines in Figures 1-3) in the inner stellar ejecta isotopes made in the pre-CCSN phase are mostly destroyed to make new species, due to extreme temperatures and densities (see Fig. 4). Explosive Si-burning mostly feeds the pro-

1 Full data of these models can be found at: https://ccsweb.lanl.gov/astro/nucleosynthesis/nucleosynthesis_astro.html 


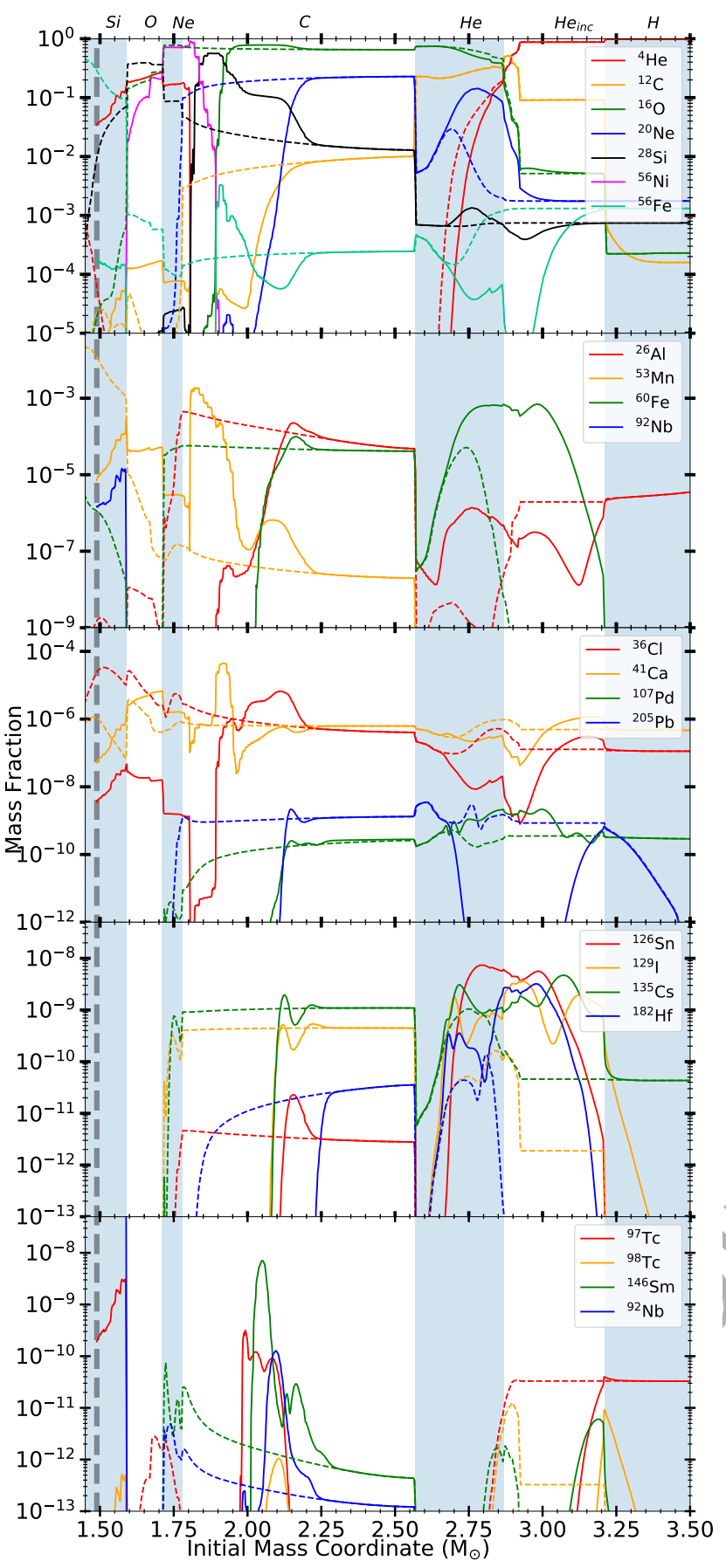

Figure 1. Abundance profile (in mass fraction) of selected isotopes for a selected $15 \mathrm{M}_{\odot}$ model with mass cut of $1.50 \mathrm{M}_{\odot}$ (the lowest of the $15 \mathrm{M}_{\odot}$ models) shown as a thick dashed vertical line and explosion energy of $4.79 \times 10^{51}$ ergs. The top panel shows isotopes that represent the pre-CCSN structure of the model, with alternating shaded and non-shaded sections showing the different burning ashes. Each burning phase is labelled at the top of the plot. The other panels show the abundance profiles of the SLRs considered in Table 1, all radioactive isotopes including the radiogenic contribution from isotopes with half-lives of less than $10^{5}$ years. The abundance profiles are plotted at both pre-CCSN (dashed line) and post-CCSN profile (solid line). A thick gray, dashed vertical line shows the mass cut, below which we do not plot the explosive profiles. ${ }^{92} \mathrm{Nb}$ is plotted in both the second from top and the bottom panels, to show its large peak in the innermost region of the ejecta.

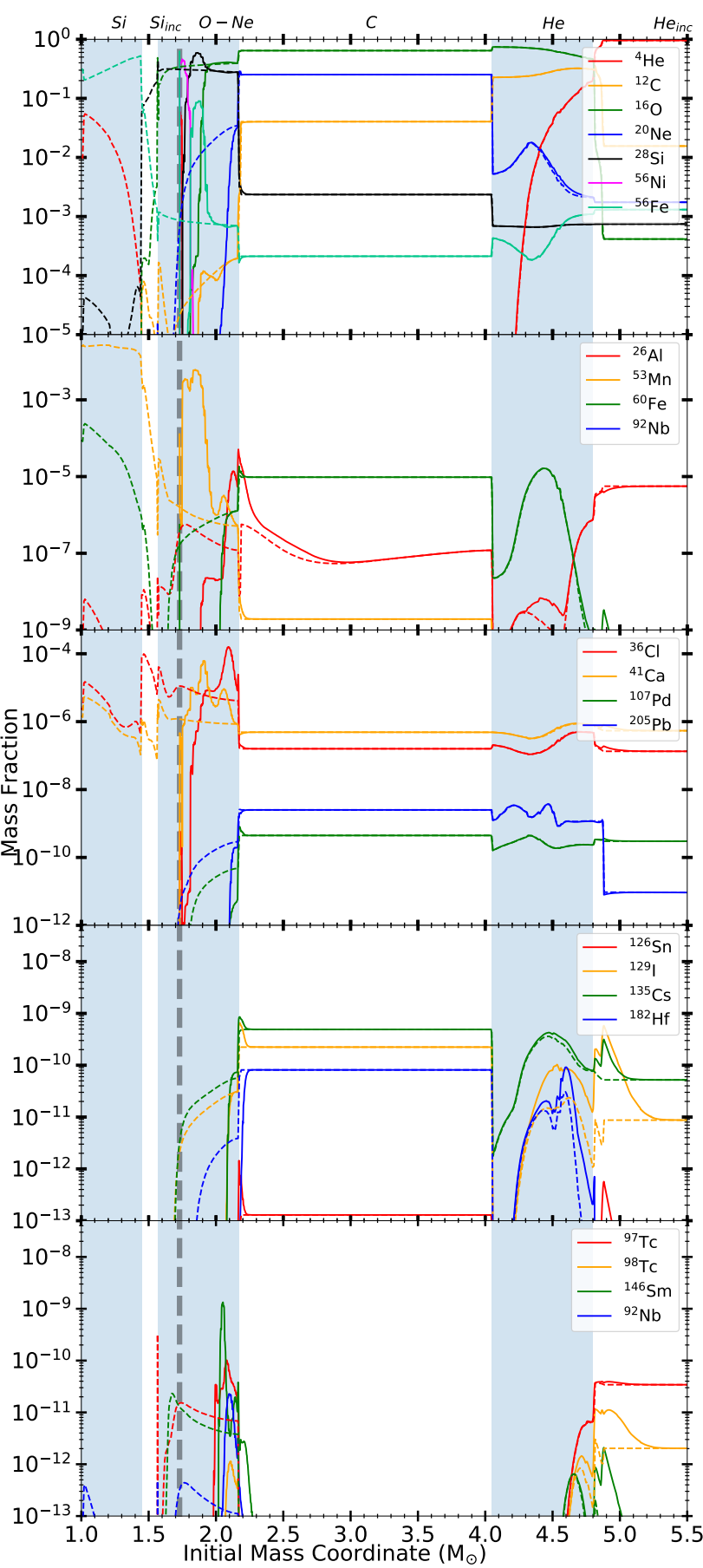

Figure 2. Same as Fig. 1 for $20 \mathrm{M}_{\odot}$ progenitor, with mass cut $1.74 \mathrm{M}_{\odot}$ and explosion energy $2.85 \times 10^{51}$ ergs.

duction of ${ }^{56} \mathrm{Ni}$ and iron-group elements, with some unconsumed ${ }^{4} \mathrm{He}$. This can be seen in Fig. 1, below $1.8 \mathrm{M}_{\odot}$. The $\alpha$-rich freeze-out can also trigger the nucleosynthesis of elements heavier than iron by charged particle reactions, up to the mass region of $\mathrm{Sr}$ and $\mathrm{Zr}$ (e.g., Woosley \& Hoffman 1992; Pignatari et al. 2016b).

As the CCSN shock expands, moving outwards in space, the peak temperature decreases (Fig. 4). Note that this temperature peak is not reached in all the structure at the same time, rather only the peak temperature is plotted 


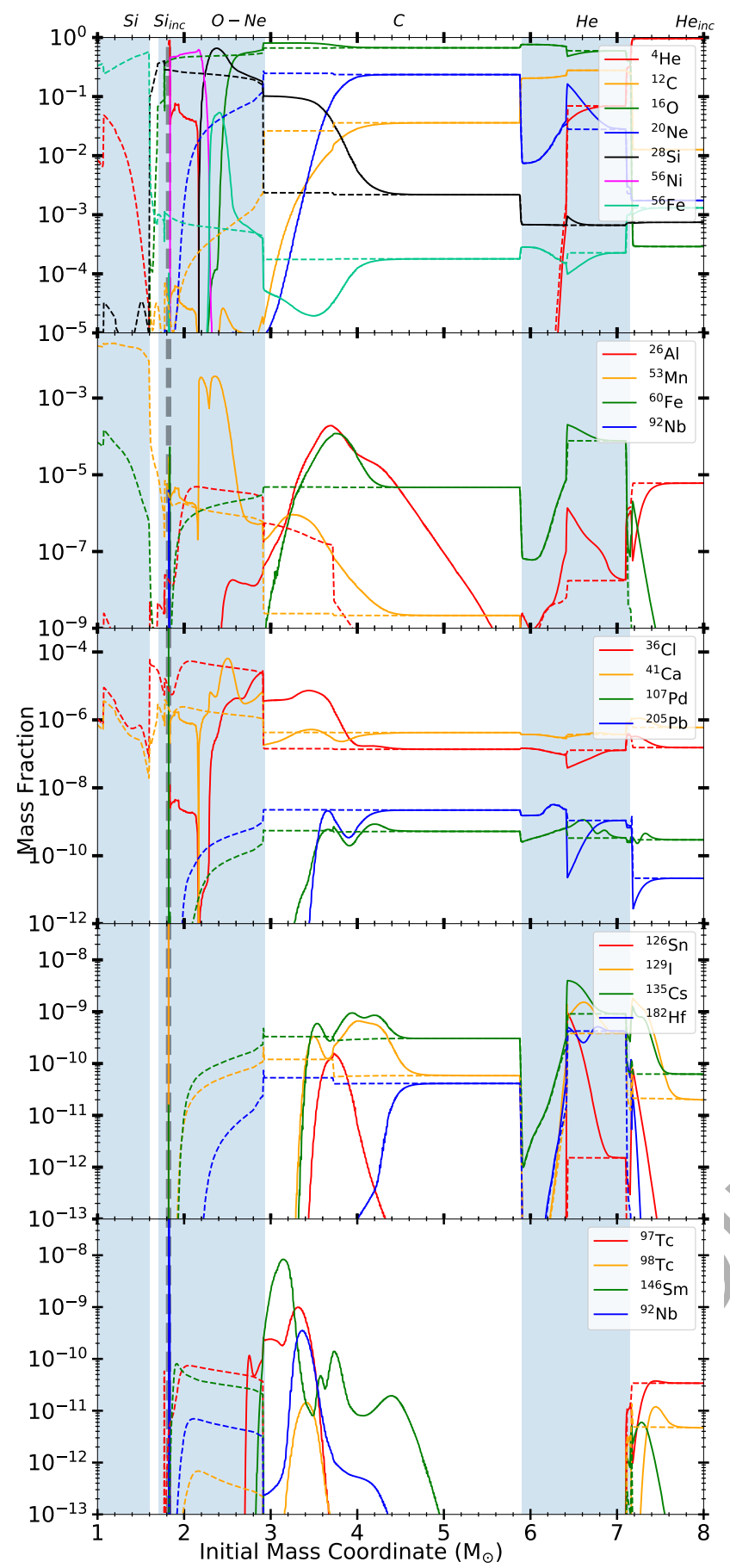

Figure 3. Same as Fig. 1 for $25 \mathrm{M}_{\odot}$ progenitor, with mass cut $1.83 \mathrm{M}_{\odot}$ and explosion energy $3.07 \times 10^{51} \mathrm{ergs}$.

per mass coordinate. Between $\sim 1.7 \mathrm{M}_{\odot}$ and $\sim 1.85 \mathrm{M}_{\odot}$ the temperatures decreases to $\sim 5$ GK and explosive Si-burning occurs (top panel of Fig. 1) (Woosley \& Weaver 1995). This consumes ${ }^{28} \mathrm{Si}$ and produce mostly ${ }^{56} \mathrm{Ni}$ and iron-group elements under nuclear statistical equilibrium, possibly leaving some ${ }^{4} \mathrm{He}$ unconsumed if the rate of expansion is sufficiently fast (Bodansky et al. 1968; Woosley et al. 1973).

Where the peak temperature of the shock falls to $\sim 3.6 \mathrm{GK}$ (between $\sim 1.85 \quad \mathrm{M}_{\odot}$ and $\sim 1.95 \quad \mathrm{M}_{\odot} \quad$ in Fig. 1), explosive O-burning products are mostly obtained
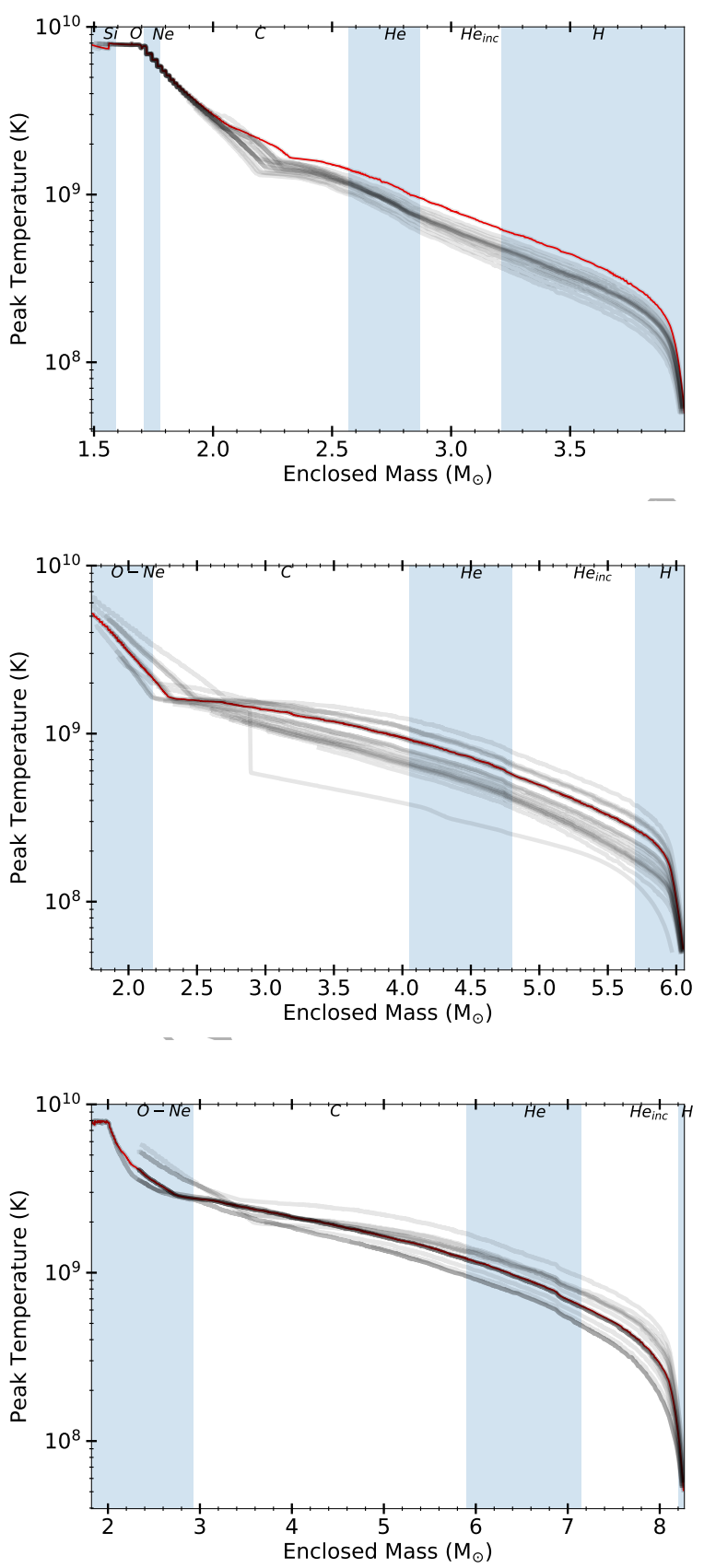

Figure 4. Peak temperature profiles of all the 62 models, constructed by selecting the maximum temperature reached at each mass coordinate as the shock passes through it. The $15 \mathrm{M}_{\odot}, 20 \mathrm{M}_{\odot}$ and $25 \mathrm{M}_{\odot}$ models are shown in the upper, middle and lower panels, respectively. Alternating shaded and non-shaded areas highlight pre-CCSN burning ashes as labelled on the top. The profiles marked in red are those of the models shown in Fig.1 - Fig.3.

(Truran \& Arnett 1970; Woosley et al. 1973). In the figure, this consumes ${ }^{16} \mathrm{O}$ and produces ${ }^{28} \mathrm{Si}$.

As the peak temperature of the shock falls between $\sim 3.2 \mathrm{GK}$ and $\sim 2.5 \mathrm{GK}$ (Rapp et al. 2006) also the signature of explosive Ne-burning can be obtained (between $\sim 1.95 \mathrm{M}_{\odot}$ and $\sim 2.1 \mathrm{M}_{\odot}$ in Fig. 1). As in pre-explosive nucleosynthesis ${ }^{20} \mathrm{Ne}$ is consumed by photodisintegration and $\alpha$-captures 
(e.g., Thielemann \& Arnett 1985). Heavy isotopes provide successive $(\gamma, \mathrm{n}),(\gamma, \mathrm{p})$, and $(\gamma, \alpha)$ photodisintegration reactions, allowing for lighter seed nuclei to produce proton rich isotopes such as ${ }^{92} \mathrm{Nb}$ and ${ }^{146} \mathrm{Sm}$. This process is the $\gamma$ process (e.g. Rauscher et al. 2013; Pignatari et al. 2016a).

As the peak temperature drops to $\sim 2$ GK the explosive C-burning signature becomes dominant (between $\sim 2.1 \mathrm{M}_{\odot}$ and $\sim 2.55 \mathrm{M}_{\odot}$ in Fig. 1) (Hansen 1971; Truran \& Cameron 1978). This consumes ${ }^{12} \mathrm{C}$ left in the $\mathrm{C}$-shell ashes, modifying local pre-CCSN abundances of C-burning ashes like $\mathrm{Ne}, \mathrm{Na}$ and $\mathrm{Mg}$. The neutron density reaches $\sim 10^{18} \mathrm{~cm}^{-3}$ and the proton density reaches above $10^{20} \mathrm{~cm}^{-3}$ (see Fig. 8 of Jones et al. 2019). This allows for the nucleosynthesis of both neutron-rich and proton-rich isotopes. Above $\sim 2.2 \mathrm{M}_{\odot}$, as temperatures drop to $\sim 2 \mathrm{GK}$, the rate of the ${ }^{22} \mathrm{Ne}(\alpha, \mathrm{n}){ }^{25} \mathrm{Mg}$ neutron source reaction decreases, as such we see little to no nucleosynthesis between $2.2 \mathrm{M}_{\odot}$ and 2.55 $\mathrm{M}_{\odot}$.

As the shock expands and peak temperatures drop to $\sim 1$ GK, explosive He-burning occurs between $\sim 2.55 \mathrm{M}_{\odot}$ and $\sim 3.1 \mathrm{M}_{\odot}$ in the $15 \mathrm{M}_{\odot}$ model shown in Fig. 1. Here ${ }^{4} \mathrm{He}$ is consumed to produce isotopes in the $\alpha$-capture chain. At mass coordinate $2.77 \mathrm{M} \odot$ (the peak of ${ }^{20} \mathrm{Ne}$ production), neutrons are significantly made by ${ }^{22} \mathrm{Ne}(\alpha, \mathrm{n})^{25} \mathrm{Mg}$. The neutrons made by ${ }^{17} \mathrm{O}(\alpha, \mathrm{n}){ }^{20} \mathrm{Ne}$ and ${ }^{21} \mathrm{Ne}(\alpha, \mathrm{n}){ }^{24} \mathrm{Mg}$, are recycled from those previously captured by ${ }^{16} \mathrm{O}$ and ${ }^{20} \mathrm{Ne}$, respectively (e.g., Raiteri et al. 1991; Pignatari et al. 2010). The peak neutron density here is $\sim 10^{18} \mathrm{~cm}^{-3}$ and proton density is $\sim 5 \times 10^{15} \mathrm{~cm}^{-3}$ (see Fig. 8 of Jones et al. 2019). While the neutron density is similar within explosive helium burning and explosive oxygen burning, the production of neutron-rich isotopes is considerably higher in explosive helium burning, as at these lower temperatures photodisintegration reactions are not active for the heavy isotopes beyond iron.

The $20 \mathrm{M}_{\odot}$ and $25 \mathrm{M}_{\odot}$ models (Fig. 2 and Fig. 3 respectively) show some important differences in the CCSN ejecta compared to the $15 \mathrm{M}_{\odot}$ model of Fig. 1 . The $\mathrm{O}$ and Ne-burning ashes within the pre-CCSN structure of the 20 $\mathrm{M}_{\odot}$ and $25 \mathrm{M}_{\odot}$ models cannot be easily distinguished, unlike these ashes in the $15 \mathrm{M}_{\odot}$ models. They are identified only by a reduction in both ${ }^{16} \mathrm{O}$ and ${ }^{20} \mathrm{Ne}$, while ${ }^{28} \mathrm{Si}$ increases considerably more than in Ne-burning (e.g., Chieffi et al. 1998). Both the $20 \mathrm{M}_{\odot}$ and $25 \mathrm{M}_{\odot}$ models show the ashes of incomplete Si-burning (labelled as $\mathrm{Si}_{\text {inc }}$ ), characterised by the partial destruction of ${ }^{28} \mathrm{Si}$ without significant production of ${ }^{4}$ He. However, these regions will not be ejected by the CCSN explosion.

The steep temperature profile of the $20 \mathrm{M}_{\odot}$ model (see Fig. 4) causes the ignition of explosive $\mathrm{Si}$-, O-, and C-burning to be located in the inner portion of the star. Due to a higher mass cut the contribution of explosive Si-burning is less present in the final yields, with a sharp peak of ${ }^{56} \mathrm{Ni}$ reaching 0.46 mass fraction at mass coordinate $1.75 \mathrm{M}_{\odot}$ (0.01 $\mathrm{M}_{\odot}$ above the mass cut). Explosive O-burning occurs at $\sim 1.87 \mathrm{M}_{\odot}$, and explosive C-burning is located at $\sim 2.15$ $\mathrm{M}_{\odot}$. As explosive C-burning is within the Ne-burning ashes, there is less ${ }^{12} \mathrm{C}$ to be used as fuel, reducing the impact of this explosive nucleosynthesis site on the final yields of isotopes created by these conditions. As the shock reaches $\sim 1$ GK, explosive He-burning would normally begin, but as this happens in the upper C-burning ashes there is no ${ }^{4} \mathrm{He}$

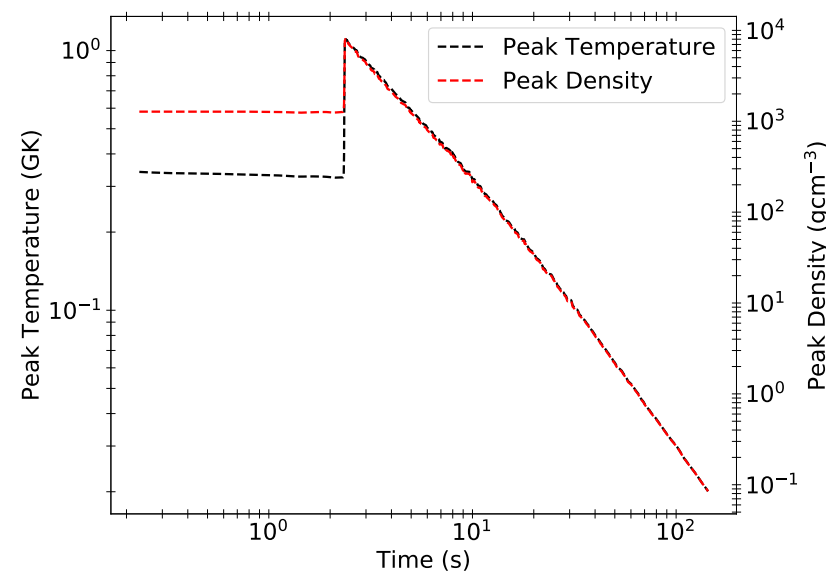

Figure 5. An example trajectory of temperature and density. Trajectory generated from mass coordinate $2.77 \mathrm{M}_{\odot}$ in model shown in Fig. 1.

to be consumed, and therefore the nucleosynthesis signature of explosive He-burning is marginal.

The $25 \mathrm{M}_{\odot}$ model shown in Fig. 3 has a structure that resembles the $15 \mathrm{M}_{\odot}$ model, however, as the mass cut is higher, there is no ejected $\alpha$-rich freeze-out material outside of the mass cut. Figure 4 shows that for all $25 \mathrm{M}_{\odot}$ models, the CCSN peak temperatures is above $1 \mathrm{GK}$ up at the bottom of the former conyective He shell, ensuring a strong activation of explosive He-burning.

To examine in detail the specific nucleosynthesis sites of SLRs in the CCSN ejecta we extracted some trajectories from the models, where we took the temperature and density profiles over the course of the CCSN event for a given mass coordinate (an example is given in Fig. 5). We then use these trajectories to examine the flux associated to each reaction and determine which are the most important for production and destruction.

\section{RESULTS}

For all SLRs we check the impact of radiogenic contributions from the decay of unstable isotopes and we include where significant. In the next section we address the nucleosynthesis of each isotope separately.

We do not consider ${ }^{7} \mathrm{Be}$ and ${ }^{10} \mathrm{Be}$ in this work as they are not produced significantly in supernovae, rather by cosmic ray spallation (Desch et al. 2004). Notice, however, that Banerjee et al. (2016) proposed that low-mass CCSNe could produce ${ }^{10} \mathrm{Be}$ by neutrino interaction with ejecta. We do not consider ${ }^{244} \mathrm{Pu}$ or ${ }^{147} \mathrm{Cm}$ as CCSN do not produce r-process isotopes.

\section{$3.1 \quad 26 \mathrm{Al}$}

In stars the ${ }^{25} \mathrm{Mg}(\mathrm{p}, \gamma){ }^{26} \mathrm{Al}$ reaction is the main nucleosynthesis channel to produce ${ }^{26} \mathrm{Al}$. The proton capture on ${ }^{25} \mathrm{Mg}$ is first activated in the $\mathrm{Mg}$ - $\mathrm{Al}$ chain during hydrogen burning in the pre-CCSN phase. In more advanced burning stages, ${ }^{26} \mathrm{Al}$ can be made during C-burning because of the combined presence of abundant ${ }^{25} \mathrm{Mg}$ and protons, where protons are made directly by $\mathrm{C}$-fusion (via the ${ }^{12} \mathrm{C}\left({ }^{12} \mathrm{C}, \mathrm{p}\right){ }^{23} \mathrm{Na}$ 
reaction). During O-fusion ${ }^{25} \mathrm{Mg}$ is destroyed to make heavier elements, and therefore these conditions are not suited for the production of ${ }^{26} \mathrm{Al}$. Similar considerations can be made for explosive nucleosynthesis triggered by the CCSN shock. The dominant depletion channels for ${ }^{26} \mathrm{Al}$ under explosive conditions are the (n,p) and (n, $\alpha)$ neutron capture reactions. These reactions deplete ${ }^{26} \mathrm{Al}$ in He-burning conditions and mitigate its production during C-burning, where neutrons are released by the ${ }^{22} \mathrm{Ne}(\alpha, \mathrm{n})^{26} \mathrm{Mg}$ reaction. Above approximately $3.2 \mathrm{M}_{\odot}$ the CCSN ejecta are carrying the signature of pre-CCSN H-burning ashes (see Fig. 1). ${ }^{26} \mathrm{Al}$ abundance is of the order of a few $10^{-6}$ by mass fraction. The He-shell ashes above $2.92 \mathrm{M}_{\odot}$ only show a minor depletion with respect to these quantities. This is due to a marginal activation of the ${ }^{22} \mathrm{Ne}(\alpha, \mathrm{n})^{26} \mathrm{Mg}$ reaction, as also indicated by the negligible depletion of the s-process seed, ${ }^{56} \mathrm{Fe}$. In the $15 \odot$ model the explosive H-burning contribution is not relevant for the nucleosynthesis of ${ }^{26} \mathrm{Al}$, rather the bulk of production lies with C-burning (see Fig. 1). Instead, the neutron burst activated in the explosive shell He-burning by the CCSN shock (n-process, e.g., Blake \& Schramm 1976; Meyer et al. 2000; Pignatari et al. 2018) depletes ${ }^{26} \mathrm{Al}$ via the neutron capture channels mentioned above. Interestingly, some production of ${ }^{26} \mathrm{Al}$ also occurs during explosive He-burning. In this case, the source of protons to activate the production channel ${ }^{25} \mathrm{Mg}(\mathrm{p}, \gamma)^{26} \mathrm{Al}$ is a combination of $(\alpha, \mathrm{p})$ and $(\mathrm{n}, \mathrm{p})$ reactions.

In the $15 \mathrm{M}_{\odot}$ model the pre-CCSN largest production of ${ }^{26} \mathrm{Al}$ occurs during carbon burning, with protons provided by the ${ }^{12} \mathrm{C}\left({ }^{12} \mathrm{C}, \mathrm{p}\right){ }^{23} \mathrm{Na}$ reaction. Within a region of about $0.8 \mathrm{M}_{\odot}$ in thickness, ${ }^{26} \mathrm{Al}$ reaches values larger than $10^{-4}$ in mass fraction. The CCSN shock will deplete most of the C-burning ashes, but the region between about 2.2 and $2.55 \mathrm{M}_{\odot}$ is ejected mostly unchanged, including the ${ }^{26} \mathrm{Al}$ reservoir made before the explosion. An explosive C-burning contribution is also present at about $2.15 \mathrm{M}_{\odot}$, shown by a small increase in ejected ${ }^{26} \mathrm{Al}$ compared to the pre-CCSN abundance. No significant production of ${ }^{26} \mathrm{Al}$ is obtained in CCSN ejecta deeper than the mass coordinates where explosive C-burning is taking place.

The reaction fluxes for these conditions are shown in Fig. 6. As expected, the strongest fluxes are those related to production via ${ }^{25} \mathrm{Mg}(\mathrm{p}, \gamma)^{26} \mathrm{Al}$ and destruction by ${ }^{26} \mathrm{Al}(\mathrm{n}, \mathrm{p}){ }^{26} \mathrm{Mg}$ driven by the high neutron densities. The relevance of the other nuclear reaction channels are at least an order of magnitude smaller. As no radioactive isotopes decaying into ${ }^{26} \mathrm{Al}$ are produced to a significant level, there is no radiogenic contribution for ${ }^{26} \mathrm{Al}$.

Comparing the $20 \mathrm{M}_{\odot}$ (Fig. 2) and $25 \mathrm{M}_{\odot}$ (Fig. 3) models with the $15 \mathrm{M}_{\odot}$ model discussed above, the most remarkable difference is the smaller abundance of pre-CCSN ${ }^{26} \mathrm{Al}$ in the C-burning ashes (ejecta between 2.2 and $4 \mathrm{M}_{\odot}$, and between 2.9 and $5.9 \mathrm{M}_{\odot}$, respectively for the 20 and $25 \mathrm{M}_{\odot}$ models), This discrepancy is explained by a less efficient or inactive C-burning in the last days of stellar evolution: the ${ }^{26} \mathrm{Al}$ previously made decays to ${ }^{26} \mathrm{Mg}$ without being produced. Interestingly, in Fig. 3 we can still find ${ }^{26} \mathrm{Al}$ abundance of the order of a few $10^{-7}$ in the upper part of the $\mathrm{O}-\mathrm{Ne}$ ashes, where final pre-explosive O-burning activation is too weak to destroy the isotope. The $25 \mathrm{M}_{\odot}$ star shows a much stronger C-burning explosive nucleosynthesis com-

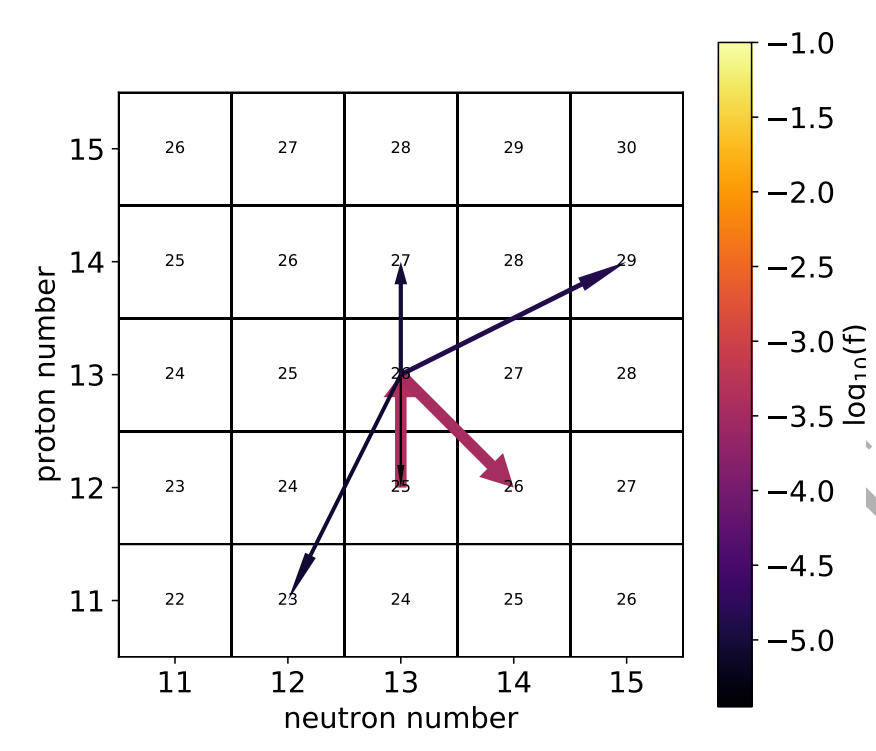

Figure 6. The distribution of fluxes $(\delta \mathrm{X} / \delta$ t, where $\mathrm{X}$ is mass fraction) for each reaction dúring explosive carbon burning at mass coordinate $2.16 \mathrm{M}_{\odot}$ in the $15 \mathrm{M}_{\odot}$ star integrated over the shock time for ${ }^{26} \mathrm{Al}$. Stellar conditions are extracted at mass coordinate $2.16 \mathrm{M}_{\odot}$ in Fig. 1. The ${ }^{26} \mathrm{Al}(\mathrm{n}, \mathrm{p}){ }^{26} \mathrm{Mg}$ and ${ }^{26} \mathrm{Al}\left(\beta^{-}\right){ }^{26} \mathrm{Mg}$ fluxes are both present as two arrows, the smaller arrow denotes the $\beta^{-}$decay.

pared to the $20 \mathrm{M}_{\odot}$ star, leading to a higher production of ${ }^{26} \mathrm{Al}$.

In the $20 \mathrm{M}_{\odot}$ model shown in Fig. 2, the peak temperature for explosive C-burning (about $2 \mathrm{GK}$ ) is reached only at the interface between the $\mathrm{O}-\mathrm{Ne}$ and $\mathrm{C}$ ashes. The same conditions are achieved in the $\mathrm{C}$-ashes for the $25 \mathrm{M}_{\odot}$ model, showing a broad production peak of ${ }^{26} \mathrm{Al}$.

In summary most of ${ }^{26} \mathrm{Al}$ in the $20 \mathrm{M}_{\odot}$ model (Fig. 2) is made by the pre-CCSN stage, in the $25 \mathrm{M}_{\odot}$ model (Fig. 3) the yields are instead mostly due to explosive nucleosynthesis. In Fig. $1{ }^{26} \mathrm{Al}$ is produced by both. From this we may already argue that the relative contribution to the total ejected ${ }^{26} \mathrm{Al}$ from different parts of the ejecta and the fraction of ${ }^{26} \mathrm{Al}$ made by the explosion do not depend solely on the initial mass of the progenitor or on the explosion energy (e.g., Limongi \& Chieffi 2006).

We examine the abundance profiles of all $15 \mathrm{M}_{\odot}$ models in Fig. 7. The top panel of Fig. 7 shows the abundance profiles of ${ }^{26} \mathrm{Al}$, and shows several similarities, with the largest variations obtained in the explosive He-burning. The yields of ${ }^{26} \mathrm{Al}$ for the $15 \mathrm{M}_{\odot}$ and $20 \mathrm{M}_{\odot}$ models show variations by a factor of about 1.5. However, due to broad production peaks during explosive He-burning the $25 \mathrm{M}_{\odot}$ models show a significantly larger variation in yield, of about an order of magnitude (see Table A1).

\section{$3.2 \quad{ }^{36} \mathrm{Cl}$ and ${ }^{41} \mathrm{Ca}$}

The radioactive isotopes ${ }^{36} \mathrm{Cl}$ and ${ }^{41} \mathrm{Ca}$ are both located next to the neutron shell closure at $\mathrm{N}=20$. In the $15 \mathrm{M}_{\odot}$ model shown in Fig. 1, the two isotopes are already efficiently pro- 

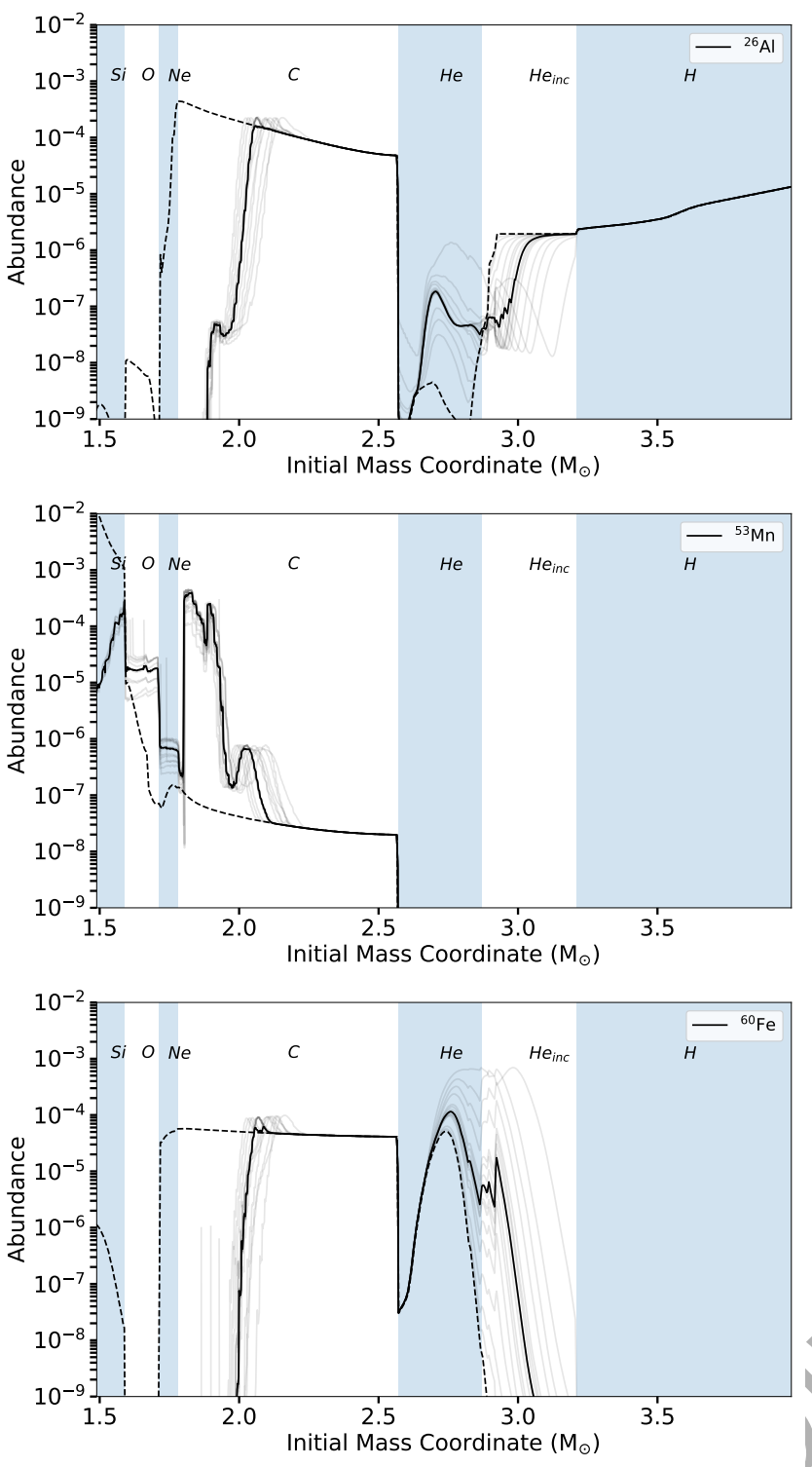

Figure 7. The isotopic abundances of ${ }^{26} \mathrm{Al},{ }^{53} \mathrm{Mn}$ and ${ }^{60} \mathrm{Ee}$ (upper panel, central panel and lower panel, respectively) are shown with respect to mass coordinate of all $15 \mathrm{M}_{\odot}$ models: pre-CCSN abundances are shown by the dashed line, the median of all models is shown by a black solid line, and thin lines show the abundance profiles for single models. Alternating shaded and non-shaded areas highlight pre-CCSN burning ashes, from left to right: $\mathrm{Si}$ ashes, $\mathrm{O}$ ashes, $\mathrm{Ne}$ ashes, $\mathrm{C}$ ashes, $\mathrm{He}$ ashes and $\mathrm{He}_{\text {inc }}$ ashes.

duced in the pre-CCSN He-burning and C-burning regions by a neutron capture on ${ }^{35} \mathrm{Cl}$ and ${ }^{40} \mathrm{Ca}$, respectively. Significant variations due to the CCSN explosion are then obtained in the calculations. In explosive nucleosynthesis ${ }^{36} \mathrm{Cl}$ is efficiently made by explosive C-burning, with a peak of production up to one order of magnitude larger than in the pre-CCSN stages. ${ }^{36} \mathrm{Cl}$ is largely destroyed from explosive O-burning and in general for all mass coordinates below $1.9 \mathrm{M}_{\odot}$. This is not the case for ${ }^{41} \mathrm{Ca}$, which can be made even in $\alpha$-rich freeze-out conditions in the deepest ejecta. The highest production peak is obtained in explosive O-burning conditions (at about $1.9 \mathrm{M}_{\odot}$ ), with abundances at least an order of magnitude higher than in the

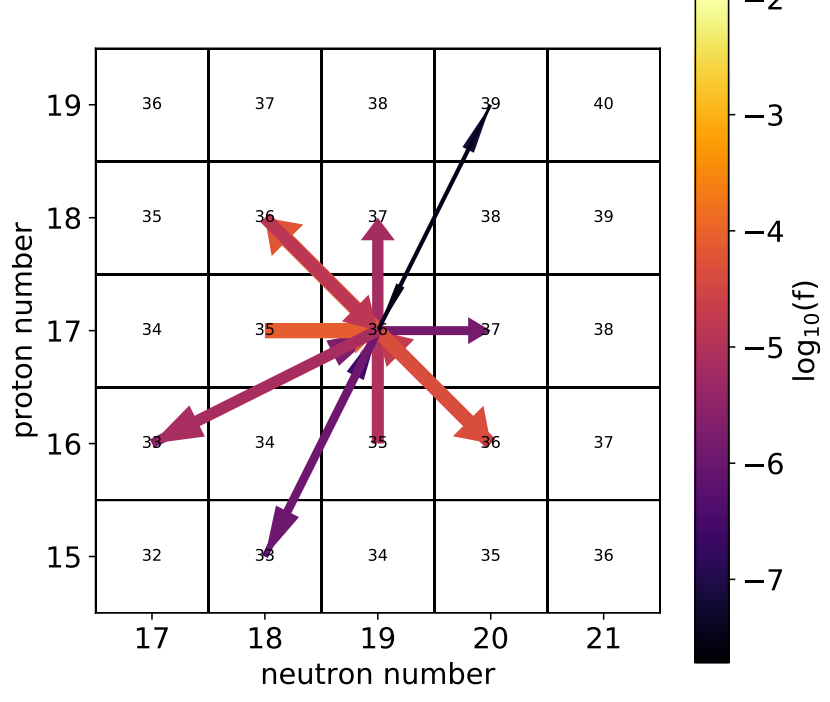

Figure 8. Same as in Fig. 6, but for ${ }^{36} \mathrm{Cl}$ in explosive carbon burning (at mass coordinate $2.1 \mathrm{M}_{\odot}$ for the $15 \mathrm{M}_{\odot}$ model in Fig. 1).

other ejected stellar layers. The dominant production channel of ${ }^{36} \mathrm{Cl}$ is ${ }^{36} \mathrm{Ar}(\mathrm{n}, \mathrm{p}){ }^{36} \mathrm{Cl}$, while it is mostly depleted by the reverse of this reaction, ${ }^{36} \mathrm{Cl}(\mathrm{p}, \mathrm{n}){ }^{36} \mathrm{Ar}$. During explosive C-burning, ${ }^{36} \mathrm{Cl}$ is produced by neutron capture on ${ }^{35} \mathrm{Cl}$ and is mostly destroyed by ${ }^{36} \mathrm{Cl}(\mathrm{n}, \mathrm{p})^{36} \mathrm{~S}$ and ${ }^{36} \mathrm{Cl}(\mathrm{p}, \mathrm{n})^{36} \mathrm{Ar}$ (see Fig. 8). ${ }^{41} \mathrm{Ca}$ is largely unaffected by explosive C-burning as the neutron capture reaction that forms ${ }^{41} \mathrm{Ca}$ is balanced by ${ }^{41} \mathrm{Ca}(\mathrm{n}, \alpha){ }^{38} \mathrm{Ar}$. In explosive He-burning both ${ }^{36} \mathrm{Cl}$ and ${ }^{41} \mathrm{Ca}$ are typically destroyed by n-process neutron captures (see Fig. 9 for ${ }^{41} \mathrm{Ca}$ ).

Comparing all the $15 \mathrm{M}_{\odot}$ models (see Fig. 10) we see that they are all qualitatively consistent for both ${ }^{36} \mathrm{Cl}$ and ${ }^{41} \mathrm{Ca}$. The largest differences in the yields are due to the amount of depletion in explosive He-burning. The location of the mass cut is another key factor in the final yields of ${ }^{41} \mathrm{Ca}$, as a high mass cut prevents the innermost portions of the star from being ejected. Examining the ejected yields from each $15 \mathrm{M}_{\odot}$ model (Table A1), ${ }^{41}$ Ca yield varies by a factor of four $\left(9.1 \times 10^{-7}-4.2 \times 10^{-6} \mathrm{M}_{\odot}\right)$, while ${ }^{36} \mathrm{Cl}$ which is not made in the deepest ejecta, varies only by $20 \%\left(8.3 \times 10^{-7}\right.$ $\left.-1.1 \times 10^{-6} \mathrm{M}_{\odot}\right)$

The pre-CCSN production of ${ }^{41} \mathrm{Ca}$ is similar between the 15,20 and $25 \mathrm{M}_{\odot}$ models. However, the production of ${ }^{36} \mathrm{Cl}$ in pre-CCSN carbon burning is higher in the $15 \mathrm{M}_{\odot}$ model (Fig.1), than in the 20 and $25 \mathrm{M}_{\odot}$ models (Figs. 2 and 3 , respectively). Post-CCSN production of ${ }^{36} \mathrm{Cl}$ in the $20 \mathrm{M}_{\odot}$ and $25 \mathrm{M}_{\odot}$ models is close to the position on the mass cut, which causes variance in the ejected yield. For ${ }^{36} \mathrm{Cl}$ the ejected yields vary by two and one order of magnitude, for $20 \mathrm{M}_{\odot}$ and $25 \mathrm{M}_{\odot}$ models respectively. For ${ }^{41} \mathrm{Ca}$ the ejected yields vary by a factor of two and by one order of magnitude, for $20 \mathrm{M}_{\odot}$ and $25 \mathrm{M}_{\odot}$ models respectively (see Table A1). 


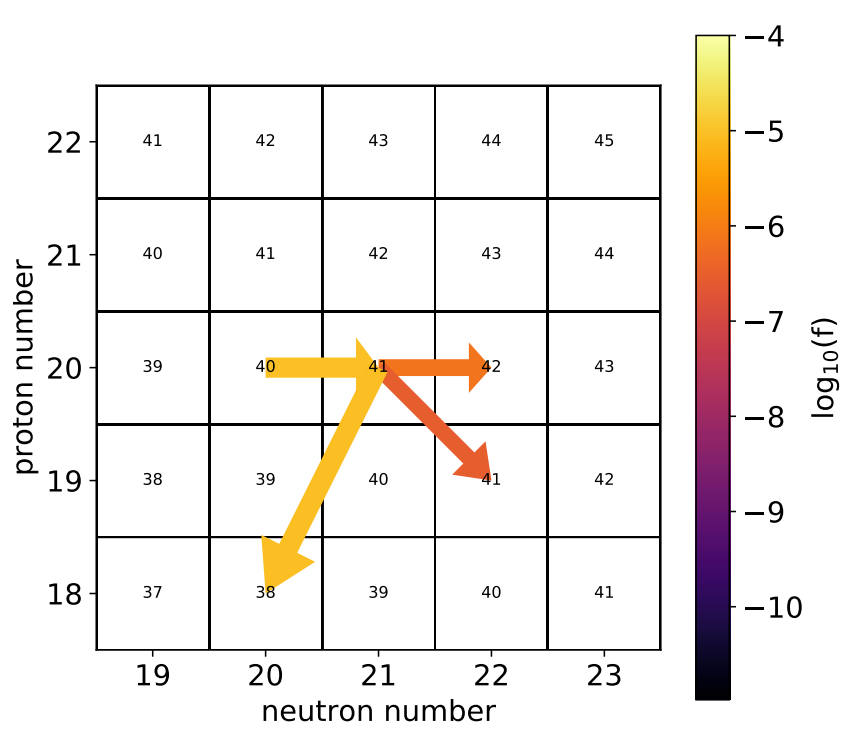

Figure 9. Same as in Fig. 6, but for ${ }^{41} \mathrm{Ca}$ in explosive helium burning (at mass coordinate $2.9 \mathrm{M}_{\odot}$ for the $15 \mathrm{M}_{\odot}$ model in Fig. 1).

\section{$3.3 \quad{ }^{53} \mathrm{Mn}$}

${ }^{53} \mathrm{Mn}$ is mostly made by explosive nucleosynthesis. In our fiducial $15 \mathrm{M}_{\odot}$ model (presented in Fig. 1), a small preCCSN component is present from the C-shell ashes, between about $1.78 \mathrm{M}_{\odot}$ and $2.54 \mathrm{M}_{\odot}$. During the CCSN explosion, additional nucleosynthesis channels contribute to most of the ejected ${ }^{53} \mathrm{Mn}$ abundance. At about $2.1 \mathrm{M}_{\odot}$, a production peak is obtained due to explosive C-burning. The largest contribution is obtained between $1.8 \mathrm{M}_{\odot}$ and $1.9 \mathrm{M}_{\odot}$, triggered by explosive O-burning and partial Si-burning. Below this point the ${ }^{53} \mathrm{Mn}$ abundance drops by three orders of magnitude in the products of complete Si-burning (between the mass cut and $1.6 \mathrm{M}_{\odot}$ ). In the deepest CCSN layers $\left(\lesssim 1.7 \quad \mathrm{M}_{\odot}\right)$ the abundance rises again due to production in $\alpha$-rich freeze-out conditions (e.g. Hoffman et al. 1996). The production peak at $\mathrm{M} \approx 1.8 \mathrm{M}_{\odot}$ is due to a near equilibrium state between the many forward and reverse reactions that are reached in explosive partial Si-burning. This produces isotopes up to the Fe-group with the largest contribution to ${ }^{53} \mathrm{Mn}$ from the decay of ${ }^{53} \mathrm{Fe}$, and its main destruction reaction being ${ }^{53} \mathrm{Mn}(\mathrm{p}, \gamma){ }^{54} \mathrm{Fe}$. During explosive Oburning a near equilibrium state is again reached at $\mathrm{M} \approx 1.9$ $\mathrm{M}_{\odot}$. The main destruction reactions are ${ }^{53} \mathrm{Mn}(\mathrm{p}, \gamma){ }^{54} \mathrm{Fe}$ and ${ }^{53} \mathrm{Mn}(\gamma, \mathrm{p}){ }^{52} \mathrm{Cr}$, and the main production reactions are the reverse of these reactions.

The other smaller peak of production is due to explosive C-burning, taking place at $\mathrm{M} \approx 2.1 \quad \mathrm{M}_{\odot}$ (Fig. 1). Here the $\alpha$-capture reaction ${ }^{24} \mathrm{Mg}(\alpha, \mathrm{p})^{27} \mathrm{Al}$ produces a large amount of protons, leading to the major production channels of ${ }^{53} \mathrm{Mn}$ : the proton-capture reactions ${ }^{53} \mathrm{Cr}(\mathrm{p}, \mathrm{n}){ }^{53} \mathrm{Mn}$ and ${ }^{52} \mathrm{Cr}(\mathrm{p}, \gamma){ }^{53} \mathrm{Mn}$. The major destruction channels are the neutron-capture reactions ${ }^{53} \mathrm{Mn}(\mathrm{n}, \mathrm{p}){ }^{53} \mathrm{Cr}$ and ${ }^{53} \mathrm{Mn}(\mathrm{n}, \gamma){ }^{54} \mathrm{Mn}$ (see Fig. 11).

The middle panel in Fig. 7 shows the ${ }^{53} \mathrm{Mn}$ abundance
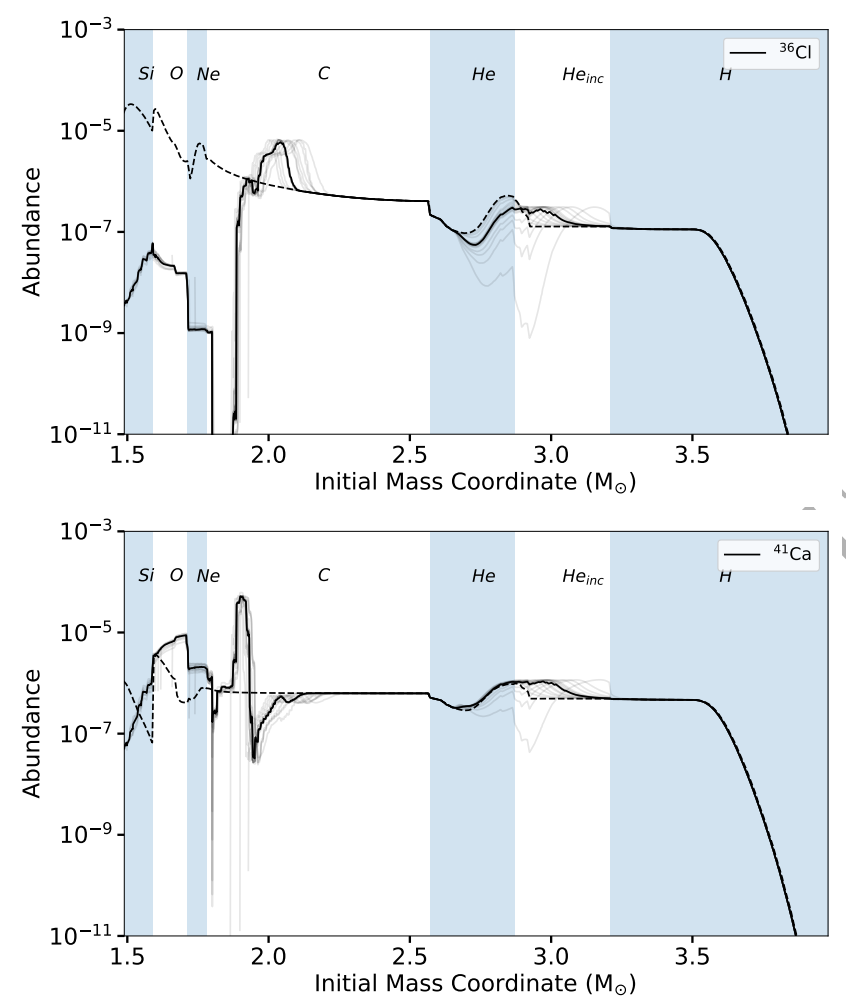

Figure 10. Same as Fig. 7. Isotopes shown are ${ }^{36} \mathrm{Cl}$ and ${ }^{41} \mathrm{Ca}$.

profiles of all the $15 \mathrm{M}_{\odot}$ models, and demonstrate that the nucleosynthesis behaves consistently across them. The yields of ${ }^{53} \mathrm{Mn}$ for the model with the highest mass cut $\left(1.9 \mathrm{M}_{\odot}\right)$ is $5.26 \times 10^{-7} \mathrm{M}_{\odot}$ while it is $5.89 \times 10^{-5} \mathrm{M}_{\odot}$ for the model with the lowest mass cut $\left(1.5 \mathrm{M}_{\odot}\right)$, a variation of the order of two orders of magnitude (see Table A2). This makes ${ }^{53} \mathrm{Mn}$ a useful diagnostic of the mass cut, as we discuss further in Section 4.1

Figs. 2 and 3 for the 20 and $25 \mathrm{M}_{\odot}$ models show a similar production pattern as described above for the $15 \mathrm{M}_{\odot}$ models, although production peaks are closer to the mass cut. This is due to production occurring in the Ne-burning ashes, with no significant production in the C-burning ashes. Examining the yields of ${ }^{53} \mathrm{Mn}$ for the 20 and $25 \mathrm{M} \odot$ models, the abundance of ${ }^{53} \mathrm{Mn}$ varies by four orders of magnitude, from $6.4 \times 10^{-9} \mathrm{M}_{\odot}$ for the model with the highest mass cut (4.89 $\left.\mathrm{M}_{\odot}\right)$ to $5.0 \times 10^{-5} \mathrm{M}_{\odot}$ with the lowest mass cut $(1.7$ $\mathrm{M}_{\odot}$ ) (Table A2). The mass cut impact is even more significant here than for the $15 \mathrm{M}_{\odot}$ model, because the models with the highest mass cut do not even eject any explosive ${ }^{53} \mathrm{Mn}$ contribution. Therefore, within the possible range of progenitor mass and explosion energy, it is indeed possible to have CCNSNe with no substantial ${ }^{53} \mathrm{Mn}$ ejected.

\section{$3.4 \quad{ }^{60} \mathrm{Fe}$}

Prior to core-collapse, ${ }^{60} \mathrm{Fe}$ is produced in C-burning and also marginally in He-burning (see the second panel from top in Fig. 1, for the reference $15 \mathrm{M}_{\odot}$ model). ${ }^{60} \mathrm{Fe}$ is destroyed in O-burning when the temperature rises above about $\sim 2 \mathrm{GK}$. During explosive nucleosynthesis all the preCCSN ${ }^{60} \mathrm{Fe}$ exposed to conditions more extreme than explo- 


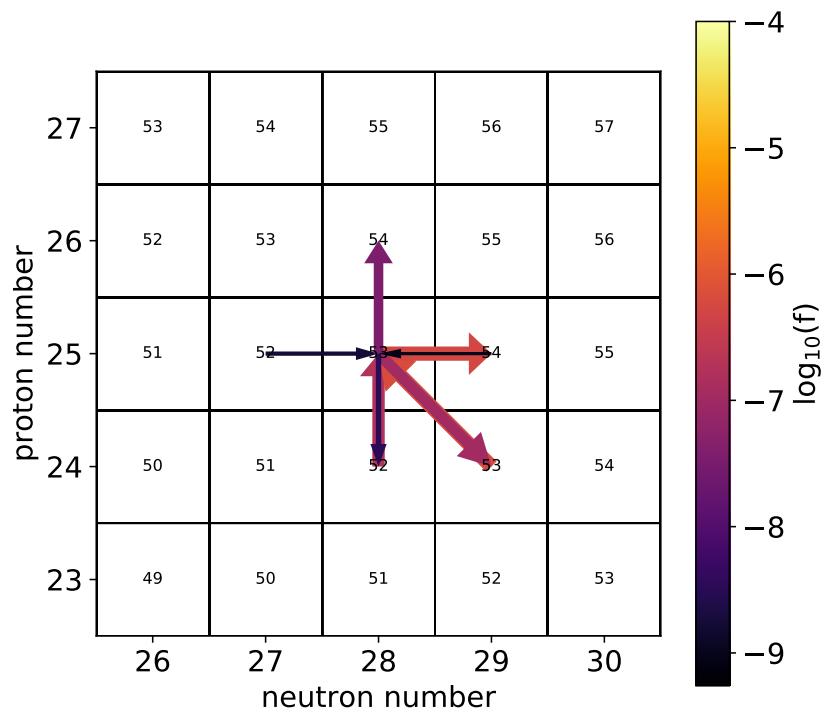

Figure 11. The same as in Fig. 6, but for ${ }^{53} \mathrm{Mn}$ from mass coordinate $2.1 \mathrm{M}_{\odot}$ in Fig. 1.

sive carbon burning is depleted. Instead, ${ }^{60} \mathrm{Fe}$ is significantly produced during explosive He-burning, as neutron densities reach $\geq 10^{18} \mathrm{~cm}^{-3}$ and neutron captures are very efficient during the n-process activation (e.g., Pignatari et al. 2018). Here, ${ }^{60} \mathrm{Fe}$ is mostly created via the double neutron-capture chain ${ }^{58} \mathrm{Fe}(\mathrm{n}, \gamma)^{59} \mathrm{Fe}(\mathrm{n}, \gamma){ }^{60} \mathrm{Fe}$, and marginally via the $\beta^{-}$decay of ${ }^{60} \mathrm{Mn}$ (see Fig. 12), made by a sequence of neutron captures starting from stable ${ }^{55} \mathrm{Mn}$. The main destruction channel of ${ }^{60} \mathrm{Fe}$ is the $(\mathrm{n}, \gamma)$ neutron capture forming ${ }^{61} \mathrm{Fe}$. The ${ }^{60} \mathrm{Fe}$ production peak due to C-burning at about 2.15 $\mathrm{M}_{\odot}$ in Fig. 1 is around one order of magnitude lower than the peak in explosive He-burning. This result may potentially be affected by parameters like the progenitor mass and the explosion energy, which we will discuss below. However, another important parameter to consider is the amount of ${ }^{22} \mathrm{Ne}$ left to produce neutrons in the $\mathrm{C}$ shell during the CCSN shock. Indeed, a weaker s-process activation in the progenitor would cause a larger remaining amount of ${ }^{22} \mathrm{Ne}$ to produce neutrons during the expløsion, a lower pre-CCSN abundance of ${ }^{60} \mathrm{Fe}$, but also a higher amount of iron seeds to feed the explosive ${ }^{60} \mathrm{Fe}$ production. Therefore, the relative relevance of explosive $\mathrm{He}$ burning and explosive $\mathrm{C}$ burning in producing ${ }^{60} \mathrm{Fe}$ could potentially vary between different sets of stellar models.

Comparing the ${ }^{60} \mathrm{Fe}$ abundance profile of the $15 \mathrm{M}_{\odot}$ model (Fig. 1) to that of the $20 \mathrm{M}_{\odot}$ (Fig. 2) and $25 \mathrm{M}_{\odot}$ (Fig. 3) models, the production sites for the radioactive isotope do not change. However, in the $25 \mathrm{M}_{\odot}$ model the relevance of explosive carbon burning is higher than for the other two masses (see peak at $3.8 \mathrm{M}_{\odot}$ in Fig. 3). In the same model, a significant pre-CCSN ${ }^{60} \mathrm{Fe}$ production is obtained in the most internal convective He shell, between about 6.4 $\mathrm{M}_{\odot}$ and $7.1 \mathrm{M}_{\odot}$. The explosion will boost further the isotope at the bottom of the shell. In the $20 \mathrm{M}_{\odot}$ model, there is no significant explosive production peak in the He-burning ashes compared to the other stellar masses considered. The

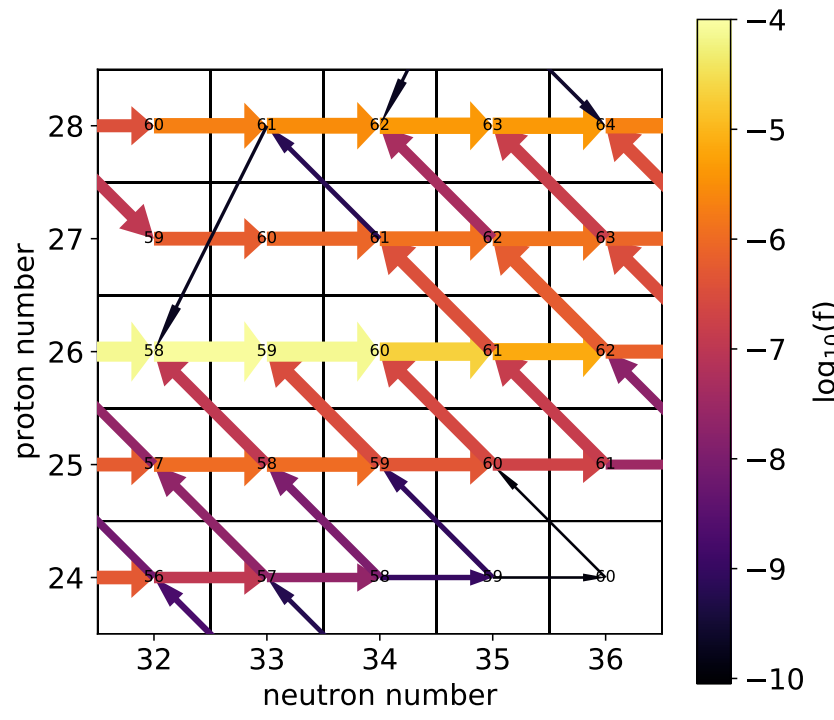

Figure 12. The same as in Fig. 6, but for ${ }^{60} \mathrm{Fe}$ from mass coordinate $3.0 \mathrm{M}_{\odot}$ in Fig. 1, respectively.

reason is that in this model the temperature peak associated the CCSN shock is too low at the bottom of the former He shell to trigger the n-process within the short explosive timescales.

The bottom panel of Fig. 7 shows the abundance profile of ${ }^{60} \mathrm{Fe}$ in all the $15 \mathrm{M}_{\odot}$ models. The qualitative behaviour is the same for all the models, with almost complete depletion for mass coordinates below explosive carbon burning and a large production in explosive He-burning. However, the amount of ${ }^{60} \mathrm{Fe}$ made by explosive He-burning changes greatly by varying the CCSN explosion parameters used in this work, with the final yield varying by two orders of magnitude for the $15 \mathrm{M}_{\odot}$ models (Table A2). This is due to the temperature variation in the He-shell material during the CCSN shock, which falls below $10^{9} \mathrm{~K}$ in the models with lower explosion energy. Due to the lack of contribution from explosive He-burning in the $20 \mathrm{M}_{\odot}$ models, the ${ }^{60} \mathrm{Fe}$ yield is lower by up to one order of magnitude relative to the 15 $\mathrm{M}_{\odot}$ model. The $25 \mathrm{M}_{\odot}$ models show an increase in yield of ${ }^{60} \mathrm{Fe}$ when compared to the $15 \mathrm{M}_{\odot}$ model, mainly due to higher contribution from the pre-CCSN stage (see also, e.g., Timmes et al. 1995; Limongi \& Chieffi 2006).

We note that there is little contribution from radiogenic decay in the final yields of ${ }^{60} \mathrm{Fe}$. For a more extended description of the production of ${ }^{60} \mathrm{Fe}$ in massive stars, we refer to Jones et al. (2019) for the same models that we use here.

\section{5 $\quad{ }^{107} \mathbf{P d}$ and ${ }^{205} \mathbf{P b}$}

Beyond iron, ${ }^{107} \mathrm{Pd}$ and ${ }^{205} \mathrm{~Pb}$ are SLRs located in-between stable isotopes $\left({ }^{106} \mathrm{Pd}\right.$ and ${ }^{108} \mathrm{Pd}$, and ${ }^{204} \mathrm{~Pb}$ and ${ }^{206} \mathrm{~Pb}$, respectively). Both SLRs areÂ̆ produced by a neutron capture on ${ }^{106} \mathrm{Pd}$ and ${ }^{204} \mathrm{~Pb}$, respectively, and they are also destroyed by neutron captures. Charged particle reactions are not relevant for the nucleosynthesis of such a heavy nuclei. the $\gamma$-process does not contribute to the production of these 

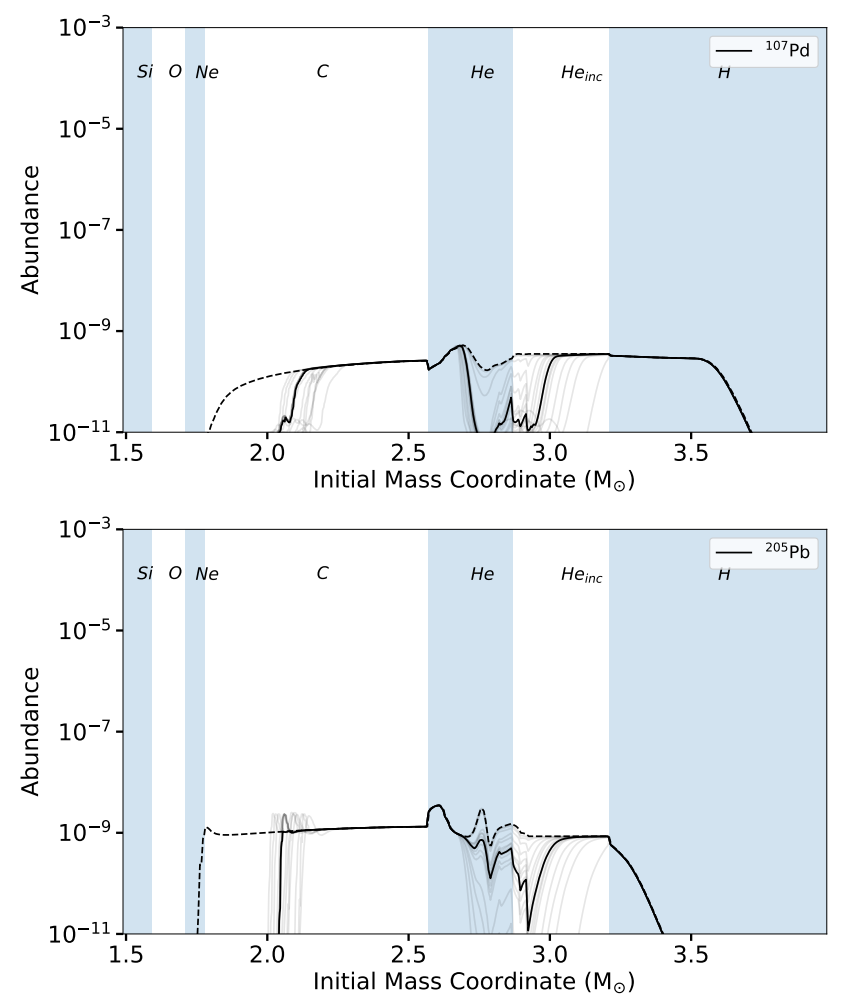

Figure 13. Same as Fig. 7. Isotopes shown are ${ }^{107} \mathrm{Pd}$ and ${ }^{205} \mathrm{~Pb}$.

isotopes either: there are no seeds for making ${ }^{205} \mathrm{~Pb}$ via photodisintegration, and ${ }^{107} \mathrm{Pd}$ is shielded from any $\gamma$-process contribution by its stable isobar ${ }^{107} \mathrm{Ag}$.

In Fig. 1 (third panel from top), ${ }^{107} \mathrm{Pd}$ and ${ }^{205} \mathrm{~Pb}$ show a relevant production by neutron captures in the pre-CCSN phase during He- and C-burning, while they are destroyed in more advanced evolutionary stages. In stellar conditions the weak decay rates of both the two isotopes depend on temperature and density. However, electron captures on ${ }^{205} \mathrm{~Pb}$ starts to affect its half-life at much lower temperatures than ${ }^{107} \mathrm{Pd}$. For instance, at typical conditions at the onset of Heburning, the half-life of ${ }^{205} \mathrm{~Pb}$ is reduced to a value of the order of a few decades, while the ${ }^{107} \mathrm{Pd} \beta$-decay half-life is still of the order of a Myr. Such a difference may also introduce significant variations in the abundance profiles of the two isotopes, as can be seen for example in the drop in abundance above $3.2 \mathrm{M}_{\odot}$ in Fig. 1 .

During the CCSN explosion both ${ }^{107} \mathrm{Pd}$ and ${ }^{205} \mathrm{~Pb}$ are destroyed below the explosive C-burning peak, at about $2.15 \mathrm{M}_{\odot}$. In explosive He-burning, the $\mathrm{n}$-process neutron burst produces ${ }^{107} \mathrm{Pd}$ by radiogenic contribution from $\beta^{-}$ decay of the neutron-rich isotopes ${ }^{107} \mathrm{Rh}$ and ${ }^{107} \mathrm{Ru}$. The pre-CCSN ${ }^{205} \mathrm{~Pb}$ is destroyed by neutron captures and cannot be formed by the $\beta^{-}$decay of unstable isotopes because it is shielded by the stable isobar ${ }^{205} \mathrm{Tl}$.

In the $20 \mathrm{M}_{\odot}$ model shown in Fig. 2, there is no effect of the explosion on the abundance profiles, as we have seen for other isotopes, due to the steep temperature gradient (Fig. 4). Comparing the $15 \mathrm{M}_{\odot}$ model (Fig. 1) with the $25 \mathrm{M}_{\odot}$ model shown in Fig. 3, the nucleosynthesis profiles are similar, with a more limited depletion of ${ }^{205} \mathrm{~Pb}$ in the 25 $\mathrm{M}_{\odot}$ model during explosive He-burning.
As a general trend, we can see from Tables A3 and A5 that the yields of ${ }^{107} \mathrm{Pd}$ and ${ }^{205} \mathrm{~Pb}$ decrease as the explosion energy increases, varying only by as much as a factor of two. This is due to the fact that by increasing the explosion energy more of the CCSN ejecta are exposed to conditions where both the two SLRs are depleted, and the explosive C-burning peak is moved outward in mass coordinate.

\section{$3.6 \quad{ }^{126} \mathrm{Sn}$}

The nucleosynthesis of ${ }^{126} \mathrm{Sn}$ requires neutron-rich conditions to drive a double neutron capture via the reaction chain ${ }^{124} \operatorname{Sn}(\mathrm{n}, \gamma){ }^{125} \mathrm{Sn}(\mathrm{n}, \gamma){ }^{126} \mathrm{Sn}$. The efficiency of this channel depends on the temperature dependent $\beta$-decay at ${ }^{125} \mathrm{Sn}$, whose half-life becomes less than a day in typical He-burning conditions and of the order of an hour or less in C-burning conditions (e.g., Takahashi \& Yokoi 1987). Therefore, high neutron densities are required to accumulate a significant amount of ${ }^{126} \mathrm{Sn}$. Figure 1 shows that in the $15 \mathrm{M}_{\odot}$ model ${ }^{126} \mathrm{Sn}$ is marginally produced in the pre-CCSN/phase in the C-burning ashes. During explosive nucleosynthesis, ${ }^{126} \mathrm{Sn}$ is produced in sites of high neutron density, specifically in explosive C burning (around 2.16 M॰) and much more significantly (by three orders of magnitude in this model) in explosive He burning $\left(2.75-3.2 \mathrm{M}_{\odot}\right)$. Thanks to the high neutron densities reached by the n-process, we notice also a small additional radiogenic contribution to ${ }^{126} \mathrm{Sn}$ by ${ }^{126} \mathrm{In}$.

In the fiducial $20 \mathrm{M} \odot$ model (shown in Fig. 2) explosive C- and He-burning are not significantly activated, resulting in a lack of a neutron source and no production of ${ }^{126} \mathrm{Sn}$. Furthermore, the pre-CCSN production of ${ }^{126} \mathrm{Sn}$ is even lower than in the $15 \mathrm{M}_{\odot}$ model, by almost two orders of magnitude. Finally, also in the $25 \mathrm{M}_{\odot}$ model in Fig. 3, $126 \mathrm{Sn}$ production occurs during the explosion, in both the C-burning (with similar abundance as in the $15 \mathrm{M}_{\odot}$ model) and He-burning ashes (but an order of magnitude lower than in the $15 \mathrm{M}_{\odot}$ model).

The final yields of the all 15, 20 and $25 \mathrm{M}_{\odot}$ models are given in Table A4. In general, the models with high explosion energies produce more ${ }^{126} \mathrm{Sn}$. This is due to an increase in neutron density in explosive C-burning, and to a broader range of ejecta affected by the n-process during explosive He-burning. We obtain the largest variation of ${ }^{126} \mathrm{Sn}$ yields for the $20 \mathrm{M}_{\odot}$ models, increasing from $8.9 \times 10^{-14} \mathrm{M}_{\odot}$ in the model with the lowest explosion energy $\left(0.5 \times 10^{51} \mathrm{ergs}\right)$ to $5 \times 10^{-10} \mathrm{M}_{\odot}$ in the model with the highest explosion energy $\left(8.9 \times 10^{51} \mathrm{ergs}\right)$.

\section{7 ${ }^{129} \mathrm{I}$ and ${ }^{135} \mathrm{Cs}$}

The neutron-rich SLRs ${ }^{129} \mathrm{I}$ and ${ }^{135} \mathrm{Cs}$ are also separated from the $\beta^{-}$-valley of stability by one unstable isotope. The radiogenic contribution from their respective neutron-rich unstable isobars can be very significant in explosive conditions, or even dominate the total CCSN ejecta.

Using the $15 \mathrm{M}_{\odot}$ model shown in Fig. 1 as an example, during the pre-CCSN stages the s-process makes both ${ }^{129} \mathrm{I}$ and ${ }^{135} \mathrm{Cs}$ by neutron capture through the branching points of ${ }^{128} \mathrm{I}$ and ${ }^{134} \mathrm{Cs}$ in the He-burning shell and the C-burning shell, while they are depleted in more advanced stages. In the CCSN explosion, the pre-CCSN abundances 
are only partially modified in the explosive C-burning peak at about $2.1 \mathrm{M}_{\odot}$. While, they are depleted by photodisintegration in the deeper parts of the former convective $\mathrm{C}$ shell. The n-process triggered by explosive He burning generates a complex pattern of production peaks. ${ }^{129} \mathrm{I}$ and ${ }^{135} \mathrm{Cs}$ are produced directly only in he mildest n-process ejecta, the highest peaks of production are given by radiogenic contribution from higher neutron-rich isotopes. For the models considered in this work, the ejected isotopes ${ }^{129} \mathrm{Te},{ }^{129} \mathrm{Sb}$, ${ }^{129} \mathrm{Sn}$ and ${ }^{129} \mathrm{In}$ will eventually decay into ${ }^{129} \mathrm{I}$. The radioactive isotopes ${ }^{135} \mathrm{Xe},{ }^{135} \mathrm{I}$ and ${ }^{135} \mathrm{Te}$ may instead contribute to the ejecta of ${ }^{135} \mathrm{Cs}$.

In the $20 \mathrm{M}_{\odot}$ model shown in Fig. 2, the explosive contribution to ${ }^{129} \mathrm{I}$ and ${ }^{135} \mathrm{Cs}$ is more limited compared to the $15 \mathrm{M}_{\odot}$ model, while a significant fraction of the pre-CCSN yields are ejected mostly unchanged from pre-CCSN abundances for material above $2.2 \mathrm{M}_{\odot}$ mass coordinate. A relevant production peak is still obtained with explosive Heburning, in particular for ${ }^{129} \mathrm{I}$. As we discussed for other SLRs before, this is due to the final n-process burst of neutrons from ${ }^{22} \mathrm{Ne}(\alpha, \mathrm{n}){ }^{25} \mathrm{Mg}$. The $25 \mathrm{M}_{\odot}$ model in Fig. 3 shows a similar pre-CCSN production as in the $15 \mathrm{M}_{\odot}$ model. However, during explosive nucleosynthesis we see a more complex production in explosive $\mathrm{C}$ burning at mass coordinates between $3.3 \mathrm{M}_{\odot}$ and $4.6 \mathrm{M}_{\odot}$, due to the diverse radiogenic contribution to both ${ }^{129} \mathrm{I}$ and ${ }^{135} \mathrm{Cs}$. Comparing the final yields within each 15, 20 and $25 \mathrm{M}_{\odot}$ sets of models, there are no significant changes (Table A4). The largest variation is obtained between the $15 \mathrm{M}_{\odot}, 20 \mathrm{M}_{\odot}$ and $25 \mathrm{M}_{\odot}$ models, within the yields of ${ }^{129} \mathrm{I}$ or ${ }^{135} \mathrm{Cs}$ increasing by factors of two, three and two, respectively.

\section{$3.8 \quad{ }^{182} \mathbf{H f}$}

${ }^{182} \mathrm{Hf}$ is produce by neutron capture, similar to ${ }^{129} \mathrm{I}$ and ${ }^{135} \mathrm{Cs}$. It may be directly produced from two neutron captures from ${ }^{180} \mathrm{Hf}$ through the unstable ${ }^{181} \mathrm{Hf}$, or as a result of radiogenic contribution via $\beta^{-}$decay from its more neutronrich unstable isobars. However, for the stellar calculations presented in this work we have used the ${ }^{181} \mathrm{Hf} \beta^{-}$decay rate by Goriely (1999), which does not take into account of experimental data by Bondarenko et al. (2002). Therefore, we underestimated the half-life of ${ }^{181} \mathrm{Hf}$. Lugaro et al. (2014) showed that the different half-life leads to an, increase of ${ }^{182} \mathrm{Hf}$ in CCSN by $7 \%$ in their $15 \mathrm{M}_{\odot}$ model and a factor of 2.6 for their $25 \mathrm{M}_{\odot}$ model.

In the $15 \mathrm{M}_{\odot}$ model shown in Fig. 1, during the preCCSN phase ${ }^{182} \mathrm{Hf}$ is directly produced via neutron capture in C-burning, and some late production is also visible in the deepest He-burning regions between mass coordinates $2.6 \mathrm{M}_{\odot}$ and $2.8 \mathrm{M}_{\odot}$. As for other neutron-rich SLRs seen in previous sections, the C-burning ashes are the deepest CCSN ejecta carrying some ${ }^{182} \mathrm{Hf}$. However, unlike these other SLRs, in this case there is no direct or radiogenic contribution from explosive C-burning feeding ${ }^{182} \mathrm{Hf}$. In explosive He-burning the production of ${ }^{182} \mathrm{Hf}$ is instead more complex, where the direct production is supplemented by radiogenic contributions to ${ }^{182} \mathrm{Hf}$. Within the models considered in this work, we can identify the contribution of ${ }^{182} \mathrm{Lu}$, ${ }^{182} \mathrm{Yb},{ }^{182} \mathrm{Tm}$ and ${ }^{182} \mathrm{Er}$.

Compared to the $15 \mathrm{M}_{\odot}$ model, the $20 \mathrm{M}_{\odot}$ model shown in Fig. 2 has a lower contribution from explosive nucleosyn- thesis, and therefore its yields are expected to be more affected by the ${ }^{181} \mathrm{Hf} \beta^{-}$decay rate used in the simulations. Within this model, ${ }^{182} \mathrm{Hf}$ is produced during the CCSN explosion only in the He-burning regions. On the other hand, the total ejecta are dominated by the pre-CCSN production in the $C$ shell ashes, between about $2.2 \mathrm{M}_{\odot}$ and $4 \mathrm{M}_{\odot}$. Also the ${ }^{182} \mathrm{Hf}$ ejecta from the $25 \mathrm{M}_{\odot}$ model are dominated by the pre-CCSN production. Comparing the final yields of the $15 \mathrm{M}_{\odot}$ set of models, there are variations of an order of magnitude (Table A5). This is due to the distribution of the CCSN peak temperatures reached within the stellar progenitor structure, where the more extreme explosion energy models reach the He burning ashes with higher temperatures (see Fig. 4), thus producing more neutrons and more radiogenic contributions to ${ }^{182} \mathrm{Hf}$. Variations in the yields of ${ }^{182} \mathrm{Hf}$ for the $20 \mathrm{M}_{\odot}$ models set is similar to that of the $15 \mathrm{M}_{\odot}$ models, varying by roughly one order of magnitude. Variations in the yields of ${ }^{182} \mathrm{Hf}$ for the $25 \mathrm{M}_{\odot}$ models are more limited, of a factor of two (Table A5).

\subsection{Heavy SLR isotopes accessible via the $\gamma$-process: ${ }^{92} \mathrm{Nb},{ }^{97} \mathbf{T c},{ }^{98} \mathbf{T c}$, and ${ }^{146} \mathrm{Sm}$}

The four SLRs isotopes ${ }^{92} \mathrm{Nb},{ }^{97} \mathrm{Tc},{ }^{98} \mathrm{Tc}$ and ${ }^{146} \mathrm{Sm}$ are all potential products of the $\gamma$ process in CCSNe, where the nucleosynthesis is mostly driven by the photodisintegration of heavier isotopes (e.g., Woosley \& Howard 1978; Arnould \& Goriely 2003; Rauscher et al. 2013; Pignatari et al. 2016b). In some of the CCSN ejecta exposed to the highest temperatures and densities, also charged particle reactions and in particular direct proton captures can be expected to be relevant for the production of ${ }^{92} \mathrm{Nb},{ }^{97} \mathrm{Tc}$ and ${ }^{98} \mathrm{Tc}$ (e.g., Hoffman et al. 1996; Lugaro et al. 2016; Travaglio et al. 2018). Moreover, both TC SLRs may receive a small neutron capture contribution fed from the available abundance of the stable isotope ${ }^{96} \mathrm{Ru}$, via the ${ }^{96} \mathrm{Ru}(\mathrm{n}, \gamma){ }^{97} \mathrm{Ru}\left(\beta^{+}\right){ }^{97} \mathrm{Tc}(\mathrm{n}, \gamma){ }^{98} \mathrm{Tc}$ chain. A small amount of ${ }^{146} \mathrm{Sm}$ can also be made by neutron capture, starting from the stable isotope ${ }^{144} \mathrm{Sm} .{ }^{92} \mathrm{Nb}$ is shielded from any radiogenic contribution from the proton-rich side of the valley of $\beta$ stability by its stable isobar ${ }^{92}$ Mo. The same applies for ${ }^{98} \mathrm{Tc}$ and its stable isobar ${ }^{98} \mathrm{Ru}$. A radiogenic contribution to ${ }^{146} \mathrm{Sm}$ can be made by the respective proton-rich unstable isobars, but also by the $\alpha$-decay channel ${ }^{154} \operatorname{Dy}(\alpha){ }^{150} \operatorname{Gd}(\alpha){ }^{146} \mathrm{Sm}$ after the $\beta$-decay radiogenic contributions to the single radioactive ${ }^{154}$ Dy and ${ }^{150} \mathrm{Gd}$.

For the $15 \mathrm{M}_{\odot}$ model in Figure 1, all the proton-rich SLRs except for ${ }^{98} \mathrm{Tc}$ are present in the pre-CCSN ashes of Ne-burning and partial O-burning. In these regions, between about $1.65 \mathrm{M}_{\odot}$ and $1.8 \mathrm{M}_{\odot}$, the $\gamma$-process is activated (e.g., Arnould 1976). Some ${ }^{92} \mathrm{Nb}$ and ${ }^{146} \mathrm{Sm}$ can also be found in the pre-CCSN C-burning ashes up to a mass coordinate 2.55 $\mathrm{M}_{\odot}$, due to the interaction between the former convective O-burning shell and C-burning shell (e.g., Meakin \& Arnett 2006; Ritter et al. 2018; Andrassy et al. 2020). ${ }^{97} \mathrm{Tc},{ }^{98} \mathrm{Tc}$, and ${ }^{146} \mathrm{Sm}$ are also produced in the top layers of the Heburning ashes (above $2.8 \mathrm{M}_{\odot}$ in the figure), due to their neutron capture channels activated by the s-process in these regions.

During explosive nucleosynthesis, proton captures are active in the deepest region of the ejecta by $\alpha$-rich freeze- 

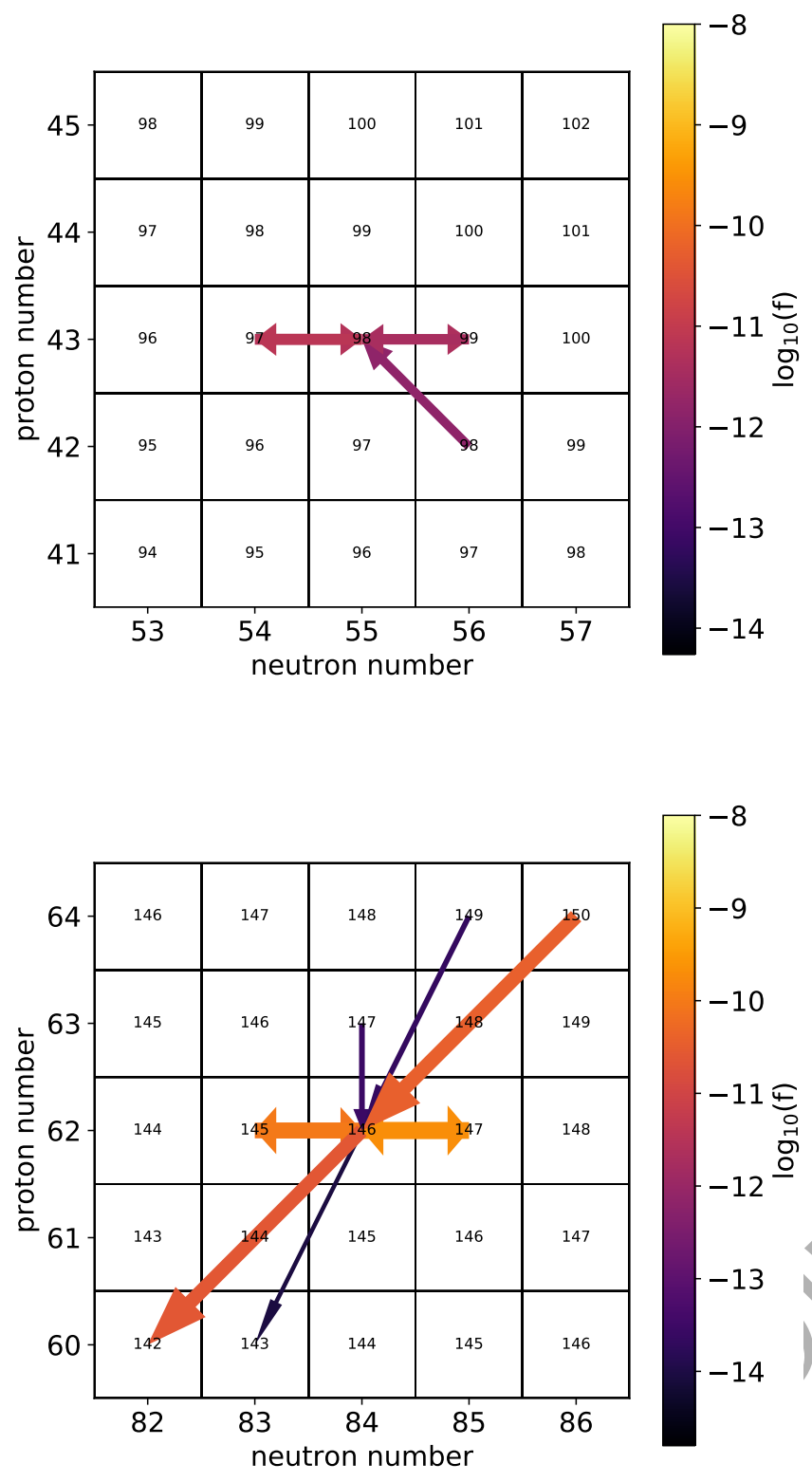

Figure 14. Same as in Fig. 6, but for ${ }^{97} \mathrm{Tc}(\mathrm{Z}=43$, top panel) and ${ }^{146} \mathrm{Sm}(\mathrm{Z}=62$, bottom panel) in explosive Ne burning (mass coordinate $2.1 \mathrm{M}_{\odot}$ from the $15 \mathrm{M}_{\odot}$ model, Fig. 1).

out (e.g., Hoffman et al. 1996; Pignatari et al. 2016b), producing ${ }^{92} \mathrm{Nb},{ }^{97} \mathrm{Tc}$ and small traces of ${ }^{98} \mathrm{Tc}$ (see in Figure 1 the region between the mass cut and $1.6 \mathrm{M}_{\odot}$ ). Note that, the abundance of ${ }^{92} \mathrm{Nb}$ is so high in this deep region that this isotope is plotted both in the second and the forth panels from the top in Figure 1. The $\gamma$-process driven by the CCSN explosion is active at a typical CCSN temperature peak between about $2.5 \mathrm{GK}$ and $3.2 \mathrm{GK}$ (e.g., Rapp et al. 2006). This temperature range is reached in the explosive Ne-burning and partial O-burning regions, where the isotopes discussed in this section are efficiently produced (bottom panel of Fig. 1). The production flux of ${ }^{97} \mathrm{Tc}$ and ${ }^{146} \mathrm{Sm}$ during explosive Ne-burning is shown in Fig. 14. In the figure we can see that together with the nucleosynthesis flow triggered by photodisintegration reactions, for ${ }^{97} \mathrm{Tc}$ also some proton capture reactions happen: namely, ${ }^{96} \mathrm{Mo}(\mathrm{p}, \gamma){ }^{97} \mathrm{Tc}$. Not included in the figure, ${ }^{91} \mathrm{Zr}(\mathrm{p}, \gamma)^{92} \mathrm{Nb}$ and ${ }^{98} \mathrm{Mo}(\mathrm{p}, \mathrm{n}){ }^{98} \mathrm{Tc}$ drives the relevant production flow for ${ }^{92} \mathrm{Nb}$ and ${ }^{98} \mathrm{Tc}$, respectively. Instead, in this same mass region ${ }^{146} \mathrm{Sm}$ is mostly driven by $(\gamma, \mathrm{n})$ and $(\gamma, \alpha)$ photodisintegration. During the explosion most of the pre-CCSN ${ }^{97} \mathrm{Tc}$, ${ }^{98} \mathrm{Tc}$ and ${ }^{146} \mathrm{Sm}$ made in the He ashes are destroyed by neutron captures. However, some of these isotopes are made again in the milder n-process components between $3.1 \mathrm{M}_{\odot}$ and $3.3 \mathrm{M}_{\odot}$, during the neutron burst.

Figure 15 shows the abundance profiles of ${ }^{92} \mathrm{Nb},{ }^{97} \mathrm{Te}$, ${ }^{98} \mathrm{Tc}$ and ${ }^{146} \mathrm{Sm}$ for all the $15 \mathrm{M}_{\odot}$ models. Qualitatively, the behaviour is consistent between the models. Except for ${ }^{92} \mathrm{Nb}$, significant variations in the abundances are seen in explosive He-burning, due to the n-process efficiency varying between the models. The yield of ${ }^{92} \mathrm{Nb}$ is mostly sensitive to the amount of CCSN fallback of the model, due to production via $\alpha$-rich freeze-out in the innermost regions. For instance, by comparing the yields of ${ }^{92} \mathrm{Nb}$ from the $15 \mathrm{M}_{\odot}$ models with a low and high amount of CCSN fallback, the ${ }^{92} \mathrm{Nb}$ abundance changes by five orders of magnitude (see Table A3). The yield of ${ }^{97} \mathrm{Tc}$ is less affected by the position of the mass cut compared to ${ }^{92} \mathrm{Nb}$, as ${ }^{97} \mathrm{Tc}$ is produced in both the $\alpha$-rich freeze-out and by neutron capture in He-burning conditions. According to Table A3, the ${ }^{97}$ Tc ejected yield varies by an order of magnitude, depending on the position of the mass cut.

From comparing the $20 \mathrm{M}_{\odot}$ (Fig. 2) and $25 \mathrm{M}_{\odot}$ (Fig. 3) models with the $15 \mathrm{M}_{\odot}$ model (Fig. 1), the first relevant difference is that the higher mass progenitors do not eject any material exposed to $\alpha$-rich freeze-out conditions. In the 20 $\mathrm{M}_{\odot}$ model, the $\gamma$-process production is limited to between mass coordinates $2 \mathrm{M}_{\odot}$ and $2.2 \mathrm{M}_{\odot}$. The milder explosive He-burning experienced in this model with respect to the 15 $\mathrm{M}_{\odot}$ model, causes a weaker efficiency of the n-process. These conditions favour the production of ${ }^{98} \mathrm{Tc}$ and ${ }^{146} \mathrm{Sm}$ in the ejecta between $4.8 \mathrm{M}_{\odot}$ and $5.2 \mathrm{M}_{\odot}$. The s-process component of ${ }^{97} \mathrm{Tc}$ made during the pre-CCSN phase appears to be more relevant than the $\gamma$-process in the ejecta of this model. In the $25 \mathrm{M}_{\odot}$ star shown in Fig. 3, the $\gamma$-process production becomes extremely important for all the SLR isotopes considered here. For instance, in this model ${ }^{97} \mathrm{Tc}$ is mostly made in these conditions (around $3.4 \mathrm{M}_{\odot}$ ). For ${ }^{146} \mathrm{Sm}$, there is also a clear contribution from explosive C-burning made by neutron captures, above mass coordinate $3.6 \mathrm{M}_{\odot} .{ }^{98} \mathrm{Tc}$ shows a comparable production due to photodisintegration and by neutron captures.

The yields of all 15, 20 and $25 \mathrm{M}_{\odot}$ models are listed in Tables A2 $\left({ }^{92} \mathrm{Nb}\right), \mathrm{A} 3\left({ }^{97} \mathrm{Tc}\right.$ and $\left.{ }^{98} \mathrm{Tc}\right)$ and $\mathrm{A} 5\left({ }^{146} \mathrm{Sm}\right)$. As mentioned, ${ }^{92} \mathrm{Nb}$ shows the largest difference in yields, depending on the location of the mass cut, varying from $2.8 \times 10^{-12} \mathrm{M}_{\odot}$ to $5.2 \times 10^{-7} \mathrm{M}_{\odot}$ in the $15 \mathrm{M}_{\odot}$ models. The $20 \mathrm{M}_{\odot}$ models have no inclusion of the $\alpha$-rich freeze-out in ejecta, and some of these models do not eject the results of explosive carbon burning either. This causes the ejected yield to also vary considerably, from $2.8 \times 10^{-15} \mathrm{M}_{\odot}$ to $1.8 \times 10^{-7} \mathrm{M}_{\odot}$. The final yields of ${ }^{97} \mathrm{Tc}$ and ${ }^{98} \mathrm{Tc}$ also change with the mass cut by about an order of magnitude, as there is production in the $\alpha$-rich freeze-out that is not included in models with more CCSN fallback. As ${ }^{97} \mathrm{Tc}$ is more impacted 

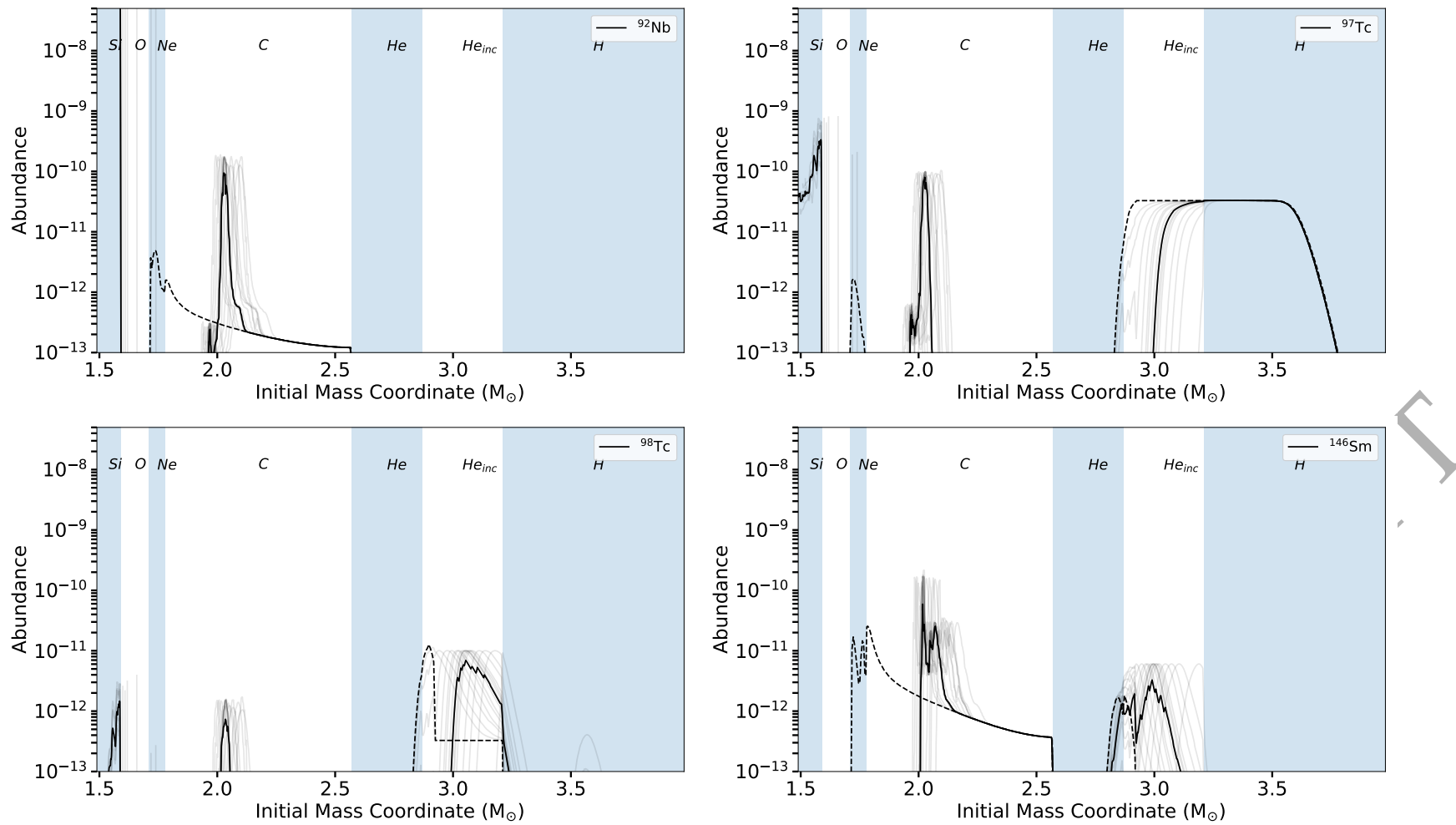

Figure 15. Abundance plots of ${ }^{92} \mathrm{Nb},{ }^{97} \mathrm{Tc},{ }^{98} \mathrm{Tc}$ and ${ }^{146} \mathrm{Sm}$ for all $15 \mathrm{M}_{\odot}$ models, as in Fig. 7. It should be noted at ${ }^{92} \mathrm{Nb}$ abundances reach peaks of about $1 \times 10^{-5}$ mass fraction in $\alpha$-rich freeze-out conditions, for mass coordinates $\mathrm{M}_{<} 1.6 \mathrm{M}_{\odot}$.

by $\alpha$-rich freeze-out its ejected yield varies by an order of magnitude in the $15 \mathrm{M}_{\odot}$ models and by a factor of two in the $20 \mathrm{M}_{\odot}$. As ${ }^{98} \mathrm{Tc}$ is less impacted it has a similar variation between models across the $15 \mathrm{M}_{\odot}, 20 \mathrm{M}_{\odot}$ and $25 \mathrm{M}_{\odot}$ models, of around a factor of two. ${ }^{146} \mathrm{Sm}$ shows more limited variation in the yield across the $15 \mathrm{M}_{\odot}$ and $25 \mathrm{M}_{\odot}$ models, up to a factor of $20 \%$. The $20 \mathrm{M}_{\odot}$ and $25 \mathrm{M}_{\odot}$ models with the mass cut above the location of explosive C-burning have reduced yields, from $8.3 \times 10^{-11} \mathrm{M}_{\odot}$ in the $20 \mathrm{M}_{\odot}$ model with the lowest mass cut to $1.0 \times 10^{-13} \mathrm{M}_{\odot}$ in the $20 \mathrm{M}_{\odot}$ model with the highest mass cut.

\section{DISCUSSION}

Starting from the analysis provided in the previous section, we discuss here the possibility of using SLRs abundances to track the contribution of the different components in the CCSN ejecta. We then compare our results with other stellar yield sets available in the literature.

\subsection{Abundances of SLR isotopes: a diagnostic for CCSN nucleosynthesis?}

In Section 3, we have described in detail the nucleosynthesis of SLRs in the different components of CCSN ejecta and have shown that the same SLR can be found in different parts of the ejecta. In some cases (e.g., ${ }^{36} \mathrm{Cl}$ and ${ }^{41} \mathrm{Ca}$ ) the nucleosynthesis production paths change from one region to the other, with different nuclear reactions relevant for the production in the different regions. In other cases, the important nuclear reactions are the same (e.g., ${ }^{60} \mathrm{Fe}$ ), but they are triggered at different temperature and density conditions. Furthermore, the stellar half-life of most of SLRs is long enough to have a significant amount of their pre-CCSN production still present in the final ejecta (e.g., ${ }^{26} \mathrm{Al}$ and $\left.{ }^{182} \mathrm{Hf}\right)$. Overall six main nucleosynthesis components in CCSN ejecta can be identified from our analysis for the SLRs: (1) $\alpha$-rich freeze-out; (2) explosive Si-burning; (3) explosive O-burning; (4) explosive Ne-burning with partial O-burning; (5) explosive C-burning together with preCCSN C-burning ashes; and (6) the most external ejecta with He-burning and H-burning products, from both preCCSN and explosive nucleosynthesis. The production of isotopes in H-burning and He-burning conditions differ from each other, although, depending on the evolution history of the progenitor star, it is possible to find significant overlap between the ejecta of these two components. Additionally, a precise discussion of the SLR production in the He-burning region requires the He-burning ejecta to be split into subcomponents. Therefore, for the sake of simplicity we consider these most external components together.

In Fig. 16, as a summary, we show the $15 \mathrm{M}_{\odot}$ CCSN ejecta, highlighting the main nucleosynthesis components for the different SLRs. Using the simplified scheme of the six components mentioned above, the components can be roughly divided as described in the caption of Fig. 16.

The $\alpha$-rich freeze-out component (1) is present in most of the $15 \mathrm{M}_{\odot}$ CCSN models of our set, and in one model of the $20 \mathrm{M}_{\odot}$ star, as the inner most regions of most models are swallowed by the mass cut. Although our models tend to have the $\alpha$-rich freeze-out component in smaller progenitor 


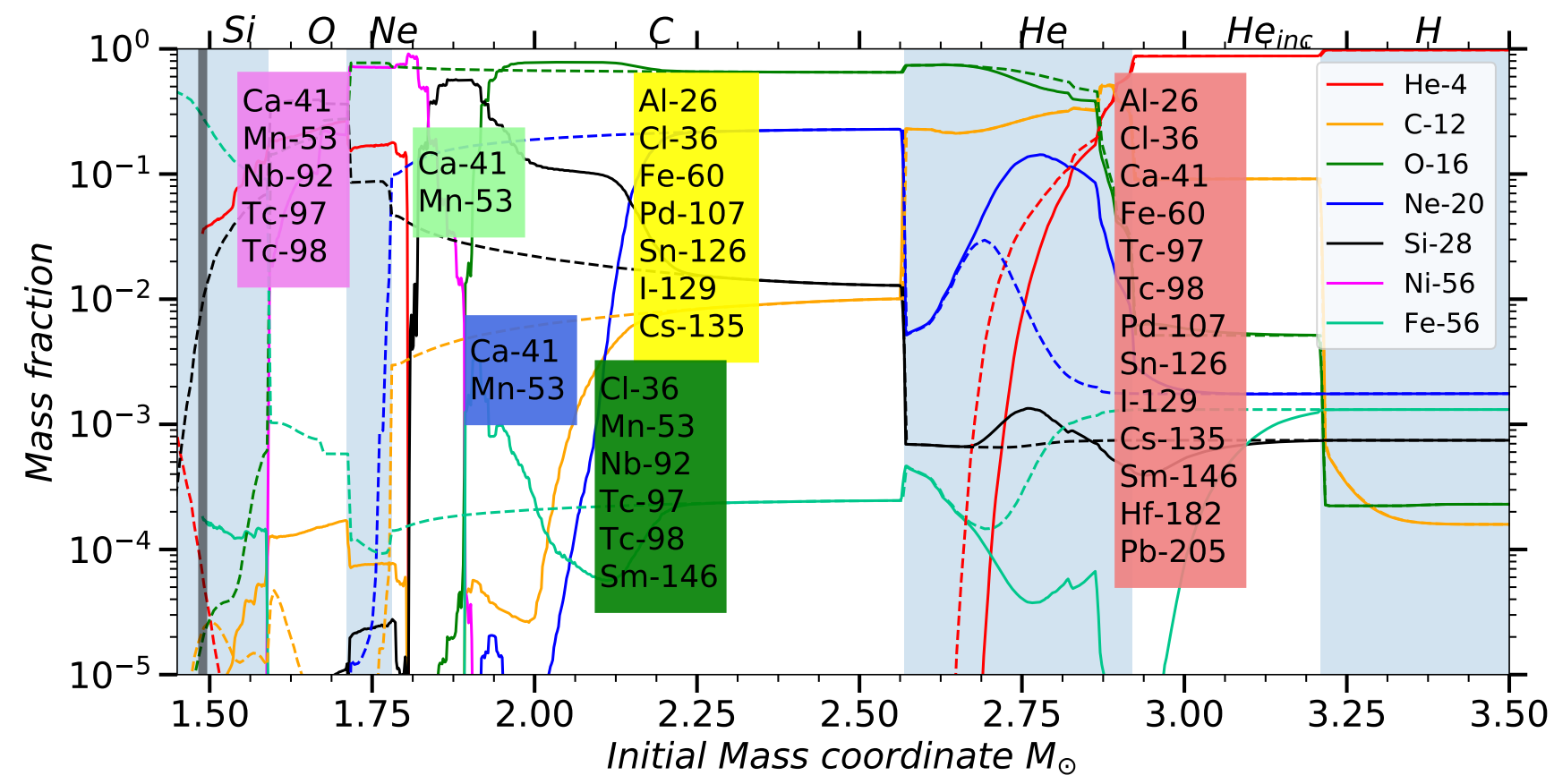

Figure 16. As a summary, we highlight the main production layers in the CCSN ejecta for the each SLR. We use as a background structure the $15 \mathrm{M}_{\odot}$ model (shown in the top panel of Fig. 1). Each colour defines a specific explosive nucleosynthesis regime: pink is $\alpha$-rich freeze-out $\left(1.5 \mathrm{M}_{\odot}\right.$ (location of the mass cut) $\left.\lesssim \mathrm{M}_{\odot} \lesssim 1.67 \mathrm{M}_{\odot}\right)$, light green explosive Si-burning $\left(1.67 \mathrm{M}_{\odot} \lesssim \mathrm{M}_{\odot} \lesssim 1.82 \mathrm{M}_{\odot}\right)$, blue explosive O-burning $\left(1.82 \mathrm{M}_{\odot} \lesssim \mathrm{M}_{\odot} \lesssim 1.95 \mathrm{M}_{\odot}\right)$, dark green explosive Ne-burning $\left(1.95 \mathrm{M}_{\odot} \lesssim \mathrm{M}_{\odot} \lesssim 2.1 \mathrm{M}_{\odot}\right)$, yellow explosive C-burning $\left(2.1 \mathrm{M}_{\odot} \lesssim \mathrm{M}_{\odot} \lesssim 2.55 \mathrm{M}_{\odot}\right)$, and red explosive He-burning $\left(\mathrm{M}_{\odot} \gtrsim 2.55 \mathrm{M}_{\odot}\right)$, The position of each box does not depict the exact mass range of nucleosynthesis, rather it is indicative of the rough position.

masses, there is no clear correlation with the progenitor mass or linear dependence with explosion energy. In the following section, we will see that in other stellar sets this component is not ejected from any stellar masses. We have seen that the SLR isotopes ${ }^{92} \mathrm{Nb}$ and ${ }^{97} \mathrm{Tc}$ are efficiently made by component (1), together with a contribution from the explosive Ne-burning, component (4), among others. Our ${ }^{97} \mathrm{Tc}$ abundances are not particularly enhanced compared to other stellar sets without $\alpha$-rich freeze-out in $15 \mathrm{M}_{\odot}$ stars (see following section). This is because the ${ }^{97} \mathrm{Tc}$ production by component (1) is less than an order of magnitude larger than by produced component (4), with additional contributions from explosive C-burning (5) and He-burning (6), respectively. Therefore, only the ${ }^{92} \mathrm{Nb}$ abundance can be used as a diagnostic of component (1) contribution, as it dominates its production by component (4) by up to several orders of magnitudes. An important caveat to keep in mind is that one-dimensional CCSN models tend to under-produce the $\gamma$-process production in the ${ }^{92} \mathrm{Nb}$ region by about an order of magnitude, when compared to the abundances of stable Mo and Ru proton-rich isotopes in the Solar System. This puzzle is still a matter of debate (e.g., Travaglio et al. 2018). However, the production of ${ }^{92} \mathrm{Nb}$ is so high in zone (1) of our models, that the diagnostic power of the SLR for this component can be safely derived.

The SLRs ${ }^{26} \mathrm{Al},{ }^{36} \mathrm{Cl},{ }^{41} \mathrm{Ca},{ }^{60} \mathrm{Fe},{ }^{107} \mathrm{Pd}$ and ${ }^{205} \mathrm{~Pb}$ are made by multiple components (see Figure 16), both from pre-CCSN and explosive nucleosynthesis. This makes them unsuited as a diagnostic of the CCSN properties, as the impact of real physics properties, stellar uncertainties, and nuclear physics uncertainties are difficult to disentangle. ${ }^{135} \mathrm{Cs}$ may also be included in this list of SLRs as the explosive contribution during explosive He-burning causes the contribution from component (6) to match that of component (5). This impact is dependant on the explosive energy of the model in question.

We also include ${ }^{182} \mathrm{Hf}$ in this category of SLRs with limited diagnostic capability. In our models, we have seen that the CCSN production of this isotope dominates the preCCSN production due to neutron captures in (5) and (6). However, as we mentioned in Section 3.8, our models are calculated using an older stellar half-life of the branching point ${ }^{181} \mathrm{Hf}$, which reduces the s-process production of ${ }^{182} \mathrm{Hf}$ in pre-CCSN conditions (Lugaro et al. 2014). In practice, by using the updated nuclear reaction rates the pre-CCSN production will increase, while the CCSN production will not be affected by such a modification and its effective contribution to the ejected yields will be reduced. New simulations are needed using the updated nuclear reaction to establish whether ${ }^{182} \mathrm{Hf}$ can be used a diagnostic of CCSN properties.

${ }^{53} \mathrm{Mn}$ is produced by the deepest components (1) - (4). Therefore, an efficient production is obtained for different progenitor masses and over a wide range of explosion energies. Faint supernova models with high fall-back mass will not eject these internal zones (e.g., Takigawa et al. 2008; Nomoto et al. 2013), and therefore will be ${ }^{53} \mathrm{Mn}$-poor. In our stellar sample this is the case for three $25 \mathrm{M}_{\odot}$ models.

As we discussed in Section 3.9, the typical $\gamma$-process SLRs ${ }^{97} \mathrm{Tc},{ }^{98} \mathrm{Tc}$ and ${ }^{146} \mathrm{Sm}$ (by component 4 ) can also be made by neutron captures within component (6). Additionally, ${ }^{146} \mathrm{Sm}$ is also made by component (5). In our models, for all progenitor masses and explosion setups, the $\gamma$-process 
production by component (4) is by far the most efficient for ${ }^{146} \mathrm{Sm}$. Therefore, we can consider ${ }^{146} \mathrm{Sm}$ as the only safe diagnostic to track the contribution from the $\gamma$-process and this region of the ejecta.

Finally, according to our models, ${ }^{126} \mathrm{Sn}$ is a convincing diagnostic of the high neutron densities reached during the CCSN explosion by the ${ }^{22} \mathrm{Ne}(\alpha, \mathrm{n})^{25} \mathrm{Mg}$ activation within component (5) and (6). The production associated to the final neutron density is orders of magnitude larger than the pre-CCSN production, and the ${ }^{126} \mathrm{Sn}$ abundance is extremely low in models with the weakest CCSN explosion energies. This is the case for all the progenitor masses. It is less clear that the same is true for ${ }^{129} \mathrm{I}$. While for instance we can derive similar conclusions for the $25 \mathrm{M}_{\odot}$ models (Figure 3), this is less clear for the $15 \mathrm{M}_{\odot}$ and $20 \mathrm{M}_{\odot}$ models (Figures 1 and 2, respectively), where a significant pre-CCSN production is obtained in zone (5). A more detailed analysis of the ${ }^{129} \mathrm{I}$ production and a careful handling of present nuclear uncertainties may be useful to clarify the diagnostic potential of this SLR isotope.

\subsection{Comparison with other studies}

We compare our yields to other sets of CCSN models found in literature: Rauscher et al. (2002, R02), Limongi \& Chieffi (2018, L18), Sieverding et al. (2018, S18), and Curtis et al. (2019, C19). The models described in R02 are calculated using Kepler for both progenitor and explosion calculations, with an initial composition based on Anders \& Grevesse (1989) at $\mathrm{Z}=0.02$. The models described in L18 are calculated using the FRANEC code with initial composition also based on Anders \& Grevesse (1989) at $\mathrm{Z}=0.02$. The models described in S18 are calculated using the Kepler code (Weaver et al. 1978) with an initial composition based on Lodders (2003). The explosion is simulated with a piston as described in Woosley \& Weaver (1995). The models described in C19 are calculated using the Kepler code for the progenitor (Woosley \& Heger 2007) and the PUSH model for the explosion (Perego et al. 2015). These models have an initial composition as described in Lodders (2003), at $\mathrm{Z}=0.013$. We note that the $\mathrm{C} 19$ study considers only the innermost regions of the star when calculating the explosive nucleosynthesis. S18 includes all SLRs found in Table 1, while R02, L18 and C19 do not, in particular. R02 does not include ${ }^{98} \mathrm{Tc}$; L18 does not include ${ }^{97} \mathrm{Tc},{ }^{98} \mathrm{Tc},{ }^{107} \mathrm{Pd},{ }^{126} \mathrm{Sn}$, ${ }^{129} \mathrm{I},{ }^{146} \mathrm{Sm}$ and ${ }^{182} \mathrm{Hf}$; and $\mathrm{C} 19$ does not include ${ }^{36} \mathrm{Cl},{ }^{92} \mathrm{Nb}$, ${ }^{107} \mathrm{Pd},{ }^{126} \mathrm{Sn},{ }^{129} \mathrm{I},{ }^{135} \mathrm{Cs},{ }^{146} \mathrm{Sm},{ }^{182} \mathrm{Hf}$, and ${ }^{205} \mathrm{~Pb}$.

In Figure 17 we compare the yields of the SLRs listed in Table 1 to the literature yields mentioned above. For each yield data set (R02, L18, S18, and C19) we compare the yields from the same initial máss. For completion we include the yields that include radiogenic contributions.

We first examine the $15 \mathrm{M}_{\odot}$ models (upper panel of Figure 17). In our calculations, ${ }^{53} \mathrm{Mn},{ }^{97} \mathrm{Tc},{ }^{129} \mathrm{I},{ }^{135} \mathrm{Cs},{ }^{146} \mathrm{Sm}$ and ${ }^{182}$ Hf show a relevant or dominant radiogenic production, as already described in Section 3. While, the other SLRs are mostly present already in the CCSN ejecta. The interquartile range (i.e., the range between the $25^{t h}$ and $75^{t h}$ percentiles) is limited within a factor of two for most of the isotopes. Exceptions are ${ }^{92} \mathrm{Nb},{ }^{97} \mathrm{Tc}$ and ${ }^{126} \mathrm{Sn}$. In particular, the largest interquartile range is obtained for ${ }^{92} \mathrm{Nb}$, varying by of a factor of around two orders of magnitude. The lower
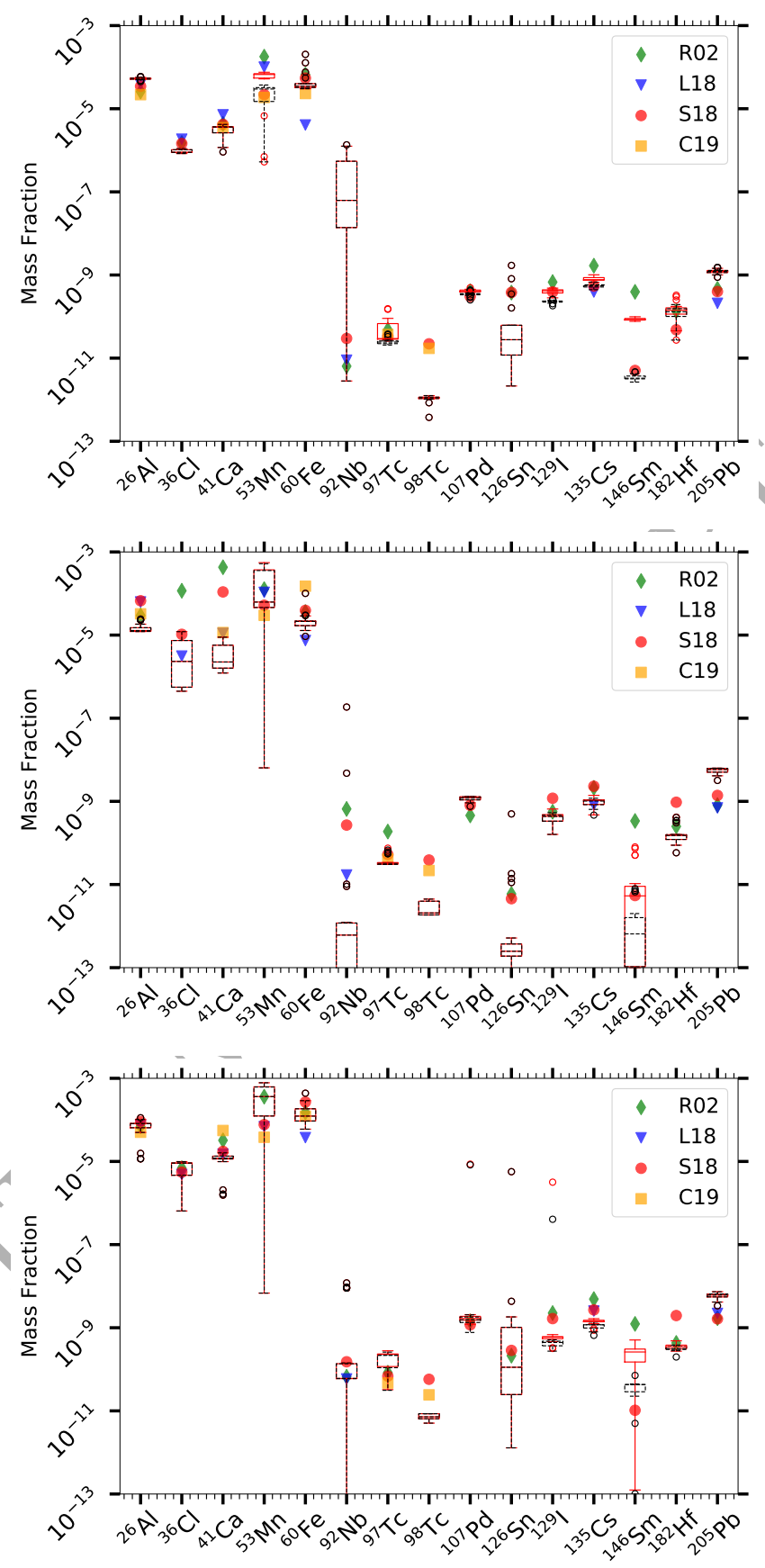

Figure 17. Comparison of all yield sets for each SLR isotope. Literature models plotted here are: Rauscher et al. (2002, R02), Limongi \& Chieffi (2018, L18), Sieverding et al. (2018, S18), and Curtis et al. (2019, C19). The upper panel shows the $15 \mathrm{M}_{\odot}$ models, the middle panel the $20 \mathrm{M}_{\odot}$ models and the lower panel the $25 \mathrm{M}_{\odot}$ models. Yields presented in this work are shown as a two boxplots, in red (yields including their respective radiogenic contributions) and in dashed black (yields not including radiogenic contributions). As by the definition of a boxplot the box size shows the range of values between the $25^{\text {th }}$ and the $75^{\text {th }}$ percentile of all the models in the dataset. The error bar on the boxplot denote the lower (upper) quartiles, and are given by subtracting (adding) to the position of the $25^{\text {th }}\left(75^{\text {th }}\right)$ percentile 1.5 times the interquartile range (i.e., the box size). All values above (below) these thresholds are plotted as outliers. Outlying models outside the outer quartiles are shown as small circles for both decayed and undecayed results, using the same color scheme as the boxes. 
quartile range can be very large towards lower abundances, where the full range of outlying models are considered within this range (see figure caption for details). ${ }^{92} \mathrm{Nb}$ is again the most remarkable case in the figure, with an effective lower quartile range of about six orders of magnitude. ${ }^{92} \mathrm{Nb}$ is effectively produced only in the innermost region of the explosion ejecta. Therefore, the models that do not include this region will have drastically lower final yields. In the figure, ${ }^{26} \mathrm{Al},{ }^{36} \mathrm{Cl},{ }^{41} \mathrm{Ca},{ }^{97} \mathrm{Tc},{ }^{107} \mathrm{Pd},{ }^{129} \mathrm{I}$ and ${ }^{182} \mathrm{Hf}$ show remarkable similarities by comparing our results with different yield sets from the literature. For other isotopes large discrepancies can be obtained.

All literature models that include ${ }^{92} \mathrm{Nb}$ show yields that lie within the lower quartile of our models, indicating a higher mass cut than many of our $15 \mathrm{M}_{\odot}$ models. Our ${ }^{53} \mathrm{Mn}$ yields lie in between the high production obtained by R02 and L18, and the lower abundances by S18 and C19. However, S18 and C19 would still be well within the production range of some of our outlying models. The ${ }^{60} \mathrm{Fe}$ yields are consistent for all models, except for L18 whose abundances are roughly one order of magnitude lower than all others. The lower ${ }^{60} \mathrm{Fe}$ abundance in L18 compared to those found in our models is likely due to a reduced neutron burst. Our ${ }^{98}$ Tc yields are about factor of 20 smaller than S18 and C19. Our models also tend to produce less ${ }^{126} \mathrm{Sn}$ compared to R02 and S18, although outlying models show similar (or even higher) production. The ${ }^{146} \mathrm{Sm}$ yields vary by two orders of magnitude between R02 and S18. Our decayed yields are in between these values, but with low variances between models. Similarly, we obtain small variations between our models for ${ }^{205} \mathrm{~Pb}$, while considering all the models plotted the range of predictions shown is about one order of magnitude. $\mathrm{Nu}$ clear and stellar physics uncertainties of different origin can be the source of these discrepancies, in particular for cases where we see little variations in our set of CCSN models.

The $20 \mathrm{M}_{\odot}$ models are shown in the central panel of Figure 17. Only for ${ }^{146} \mathrm{Sm}$ do we obtain a relevant radiogenic contribution, while this was the case for six SLRs in the 15 $\mathrm{M}_{\odot}$ models above. Boxes larger than a factor of two are obtained for ${ }^{36} \mathrm{Cl},{ }^{41} \mathrm{Ca},{ }^{53} \mathrm{Mn},{ }^{92} \mathrm{Nb},{ }^{98} \mathrm{Tc}$, and ${ }^{146} \mathrm{Sm}$. A large lower quartile range is also obtained for ${ }^{53} \mathrm{Mn}$, due to the contribution of outlying models with low concentrations of this isotope.

When we compare all the model sets we find that ${ }^{26} \mathrm{Al}$, ${ }^{41} \mathrm{Ca},{ }^{60} \mathrm{Fe},{ }^{97} \mathrm{Tc},{ }^{98} \mathrm{Tc},{ }^{107} \mathrm{Pd},{ }^{129} \mathrm{I},{ }^{135} \mathrm{Cs},{ }^{182} \mathrm{Hf}$, and ${ }^{205} \mathrm{~Pb}$ show a total range smaller than an order of magnitude. Instead, for ${ }^{36} \mathrm{Cl}$ our results are consistent with L18 and S18, but they are much smaller than R02. R02 also provided much higher yields for ${ }^{41} \mathrm{Ca}$ and ${ }^{92} \mathrm{Nb}$, although for ${ }^{92} \mathrm{Nb}$ there are outlying models in our'sample with much larger values and covering the full abundance range. Our upper interquartile limit for ${ }^{92} \mathrm{Nb}$ is one order of magnitude smaller than L18 and up to three order of magnitude smaller than R02. As for the $15 \mathrm{M}_{\odot}$ models in the upper panel of the figure, most of our módels show a smaller production of ${ }^{98} \mathrm{Tc}$ and ${ }^{126} \mathrm{Sn}$ by about an order of magnitude compared to S18 and C19. Finally, once the radiogenic contribution is taken into account, our ${ }^{146} \mathrm{Sm}$ median is consistent with $\mathrm{S} 18$, but it is almost two orders of magnitude smaller than R02.

The $25 \mathrm{M}_{\odot}$ models are shown in the lower panel of Figure 17 . We obtain relevant radiogenic contribution for ${ }^{129} \mathrm{I}$,

${ }^{135} \mathrm{Cs}$ and ${ }^{146} \mathrm{Sm}$, and we derived interquartile ranges larger than a factor of two only for ${ }^{53} \mathrm{Mn},{ }^{92} \mathrm{Nb},{ }^{126} \mathrm{Sn}$ and ${ }^{146} \mathrm{Sm}$. Large lower quartile ranges are also obtained for the same SLRs and for ${ }^{92} \mathrm{Nb}$. This is due to the efficient fallback of the $25 \mathrm{M}_{\odot}$ models in our stellar sample, where the products of e.g., explosive O-burning is often not ejected. This explanation, however, would not apply to ${ }^{126} \mathrm{Sn}$. Indeed, this SLR is produced primarily in explosive He-burning (see Section 3.6). In this case, the large variance in the ejected yield can be explained by the position of the ignition point of this burning phase. Comparing our $25 \mathrm{M}_{\odot}$ models to those in literature, we find overall a good consistency up to ${ }^{97} \mathrm{Tc}$, with some significant variation for ${ }^{53} \mathrm{Mn}$. We tend to produce less ${ }^{98} \mathrm{Tc},{ }^{129} \mathrm{I}$ and ${ }^{135} \mathrm{Cs}$ compared to other stellar sets, while for ${ }^{146} \mathrm{Sm}$ most of our data are in between R02 and S18 (with about two order of magnitude of variation between the two models).

In general, for SLRs lighter than ${ }^{60} \mathrm{Fe}$ there is agreement within an order of magnitude between different $15 \mathrm{M}_{\odot}$ and $25 \mathrm{M}_{\odot}$ models. The $20 \mathrm{M}_{\odot}$ models show a much larger variation, with up to three orders of magnitude difference for ${ }^{41} \mathrm{Ca}$. For SLRs heavier than ${ }^{60} \mathrm{Fe},{ }^{146} \mathrm{Sm}$ shows the largest variation of about two orders of magnitude, varying from S18 up to R02 yields. Other isotope yields are consistent within an order of magnitude. There are three exceptions: ${ }^{92} \mathrm{Nb}$, ${ }^{98} \mathrm{Tc}$, and ${ }^{126} \mathrm{Sn}$. For all the progenitor masses considered, in our calculations we produce about an order of magnitude less ${ }^{98} \mathrm{Tc}$ compared to other stellar sets. The same applies for ${ }^{126} \mathrm{Sn}$ if we only consider the $15 \mathrm{M}_{\odot}$ and $20 \mathrm{M}_{\odot}$ stars, although in this case we have outlying models consistent or even exceeding other published results. ${ }^{92} \mathrm{Nb}$ abundances show the most variance in ejected yields, due to the extreme sensitivity to mass cut. If we use our median yield as a reference, the $15 \mathrm{M}_{\odot}$ models overproduce the SLR isotope by at least three orders of magnitude (even if we have outlying models with low ${ }^{92} \mathrm{Nb}$ abundance). The median of the 20 $\mathrm{M}_{\odot}$ models is instead about an order of magnitude lower than the closest set (L18), while the $25 \mathrm{M}_{\odot}$ models median and other sets are in good agreement.

\section{CONCLUSIONS}

We presented the CCSN yields of 62 one-dimensional models for three progenitors with initial masses of 15, 20 and $25 \mathrm{M}_{\odot}$ and of solar metallicity $(\mathrm{Z}=0.02$, Grevesse \& Noels 1993). The impact on the nucleosynthesis of different explosion parameters is explored for each progenitor mass. This work is part of a set of studies focused on the production of radioactive isotopes in the same set of CCSNe models: Jones et al. (2019) analyzed the nucleosynthesis of ${ }^{60} \mathrm{Fe}$ and Andrews et al. (2020) focused on the production of radioisotopes that are relevant for the next generation of $\gamma$-ray astronomical observations. In this work, we study the production of short-lived radioactive isotopes (SLRs) that have been detected in the early Solar System (ESS), with half lives between 0.1 and 100 Myr. Note that compared to Jones et al. (2019) and Andrews et al. (2020), calculations have been redone to correctly take into account the ${ }^{26} \mathrm{Al}$ isomer during the explosion, resulting in an increase of the ${ }^{26} \mathrm{Al}$ yields compared to these previous data sets.

Using the CCSN models presented in this work, we explore for the first time in detail the nucleosynthesis for the 
fifteen SLRs both in the progenitor and during the CCSN explosion. In particular, we examine the main production and destruction sites for each isotope individually in the CCSN ejecta. We have identified the most favourable stellar conditions to produce each SLR for the CCSN models considered in this work. In particular, several SLRs are co-produced under the same explosive nucleosynthesis conditions in the CCSN ejecta, as summarised in Fig. 16.

We compared our results with different sets of CCSN models available in the literature and find the following:

- SLRs lighter than and including ${ }^{60} \mathrm{Fe}$ are in agreement within an order of magnitude across all masses.

- SLRs heavier than ${ }^{60} \mathrm{Fe}$ are generally consistent within an order of magnitude, excluding ${ }^{92} \mathrm{Nb},{ }^{98} \mathrm{Tc},{ }^{126} \mathrm{Sn}$, and ${ }^{146} \mathrm{Sm}$.

- Regarding ${ }^{92} \mathrm{Nb}$, the $15 \mathrm{M}_{\odot}$ models overproduce the SLR isotope by three orders of magnitude in the $15 \mathrm{M}_{\odot}$ models and by an order of magnitude in the $20 \mathrm{M}_{\odot}$ models.

- We produce an order of magnitude less ${ }^{98} \mathrm{Tc}$ than in other stellar sets.

- We produce an order of magnitude less ${ }^{126} \mathrm{Sn}$ relative to other 15 and $20 \mathrm{M}_{\odot}$ models.

- Rauscher et al. (2002) produces more ${ }^{146} \mathrm{Sm}$ than our models (up to two orders of magnitude) and Sieverding et al. (2018) produces less than our models (up to an order of magnitude).

Such variations in the production of SLRs in onedimensional CCSN models can be understood for nuclei like ${ }^{92} \mathrm{Nb}$ and ${ }^{53} \mathrm{Mn}$ due to their creation in the inner regions of the CCSN ejecta, and the impact of assumptions made in the models and stellar uncertainties. Also, typical $\gamma$-process products like ${ }^{146} \mathrm{Sm}$ and neutron-capture products like ${ }^{126} \mathrm{Sn}$ show variations well above an order of magnitude. Because our set of CCSN explosions cover a large parameter space, we were able to provide a first assessment of the impact of these differences. More detailed works are required in the future in order to understand and disentangle the source of these changes. In particular, for a number of SLRs $\left({ }^{41} \mathrm{Ca}\right.$, ${ }^{92} \mathrm{Nb},{ }^{98} \mathrm{Tc},{ }^{126} \mathrm{Sn}$, and ${ }^{146} \mathrm{Sm}$ ) other stellar sets show yields higher than our range of ejected abundances. Other sources of variations need to be carefully taken into account in future works, like e.g., nuclear reaction rate uncertainties and the use of different stellar progenitors. Future work will apply this broad nucleosynthesis analysis to observed ESS abundances, in order to determine if a single CCSN could populate the SLRs found in meteorites.

\section{ACKNOWLEDGEMENTS}

This work is supported by the ERC Consolidator Grant (Hungary) programme (RADIOSTAR, G.A. n. 724560). TVL, MP, and BKG acknowledge the financial support of NuGrid/JINA-CEE (NSF Grant PHY-1430152) and STFC (through the University of HullâẮ́s Consolidated Grant ST/R000840/1), and ongoing access to viper, the University of Hull High Performance Computing Facility. MP acknowledges the support from the "Lendület-2014" Programme of the Hungarian Academy of Sciences (Hungary). We thank the ChETEC COST Action (CA16117), supported by the European Cooperation in Science and Technology. This work was supported by the European UnionâĂŹs Horizon 2020 research and innovation programme (ChETEC-INFRA Project no. 101008324), and the IReNA network supported by US NSF AccelNet.

\section{DATA AVAILABILITY}

The data underlying this article will be shared on reasonable request to the corresponding author. 


\section{REFERENCES}

Anders E., Grevesse N., 1989, Geochimica et Cosmochimica Acta, 53, 197

Andrassy R., Herwig F., Woodward P., Ritter C., 2020, MNRAS, 491, 972

Andrews S., Fryer C., Even W., Jones S., Pignatari M., 2020, The Astrophysical Journal, 890, 35

Arnould M., 1976, A\&A, 46, 117

Arnould M., Goriely S., 2003, Physics Reports, 384, 1

Banerjee P., Qian Y. Z., Heger A., Haxton W. C., 2016, Nature Communications, 7, 1

Blake J. B., Schramm D. N., 1976, ApJ, 209, 846

Bodansky D., Clayton D. D., Fowler W. A., 1968, The Astrophysical Journal Supplement Series, 16, 299

Bondarenko V., et al., 2002, Nuclear Phys. A, 709, 3

Boss A. P., 2017, The Astrophysical Journal, 844, 113

Boss A. P., Keiser S. A., 2014, Astrophysical Journal, 788

Cameron A. G., Truran J. W., 1977, Icarus, 30, 447

Chieffi A., Limongi M., Straniero O., 1998, The Astrophysical Journal, 502, 737

Côté B., Lugaro M., Reifarth R., Pignatari M., Világos B., Yagüe A., Gibson B. K., 2019a, The Astrophysical Journal, 878, 156

Côté B., Yagüe A., Világos B., Lugaro M., 2019b, The Astrophysical Journal, 887, 213

Cromartie H. T., et al., 2020, Nature Astronomy, 4, 72

Curtis S., Ebinger K., Fröhlich C., Hempel M., Perego A., Liebendörfer M., Thielemann F.-K., 2019, The Astrophysical Journal, 870,2

Dauphas N., 2005, Nature, 435, 1203

Desch S. J., Connolly, Jr. H. C., Srinivasan G., 2004, The Astrophysical Journal, 602, 528

Fryer C. L., Woosley S. E., Hartmann D. H., 1999, The Astrophysical Journal, 526, 152

Fryer C. L., Andrews S., Even W., Heger A., Safi-Harb S., 2018, The Astrophysical Journal, 856, 63

Fujimoto Y., Krumholz M. R., Tachibana S., 2018, Monthly Notices of the Royal Astronomical Society, 480, 4025

Goriely S., 1999, A\&A, 342, 881

Grevesse N., Noels A., 1993, Origin and Evolution of the Elements, pp 205-257

Gritschneder M., Lin D. N., Murray S. D., Yin Q. Z., Gong M. N., 2012, Astrophysical Journal, 745

Hansen C. J., 1971, Astrophysics and Space Science, 14, 389

Hartmann L., BallesterosâĂŘParedes J., Bergin E. A., 2001, The Astrophysical Journal, 562, 852

Heger A., Woosley S. E., 2010, The Astrophysical Journal, 724, 341

Herant M., Benz W., Hix W. R., Fryer C. L., Colgate S. A., 1994, The Astrophysical Journal, 435, 339

Hoffman R. D., Woosley S. E., Qian Y. Z., 1996, The Astrophysical Journal, 482, 951

Huss G. R., Meyer B. S., Srinivasan G., Goswami J. N., Sahijpal S., 2009, Geochimica et Cosmochimica Acta, 73, 4922

Jones S. W., et al., 2019, Monthly Notices of the Royal Astronomical Society, 485, 4287

Limongi M., Chieffi A., 2006, The Astrophysical Journal, 647, 483

Limongi M., Chieffi A., 2018, The Astrophysical Journal Supplement Series, 237, 13

Lodders K., 2003, The Astrophysical Journal, 591, 1220

Lugaro M., et al., 2014, Science, 345, 650

Lugaro M., Pignatari M., Ott U., Zuber K., Trayáglio C., Gyürky G., Fülöp Z., 2016, Proceedings of the National Academy of Sciences of the United States of America, 113, 907

Lugaro M., Ott U., Kereszturi Á., 2018, Progress in Particle and Nuclear Physics

Meakin C. A., Arnett D., 2006, ApJ, 637, L53

Meyer B. S., Clayton D. D., 2000, Space Science Reviews, 92, 133

Meyer B. S., Weaver T. A., Woosley S. E., 1995, Meteoritics, 30, 325

Meyer B. S., Clayton D. D., The L. S., 2000, ApJ, 540, L49

Murray N., 2011, Astrophysical Journal, 729

Nomoto K., Kobayashi C., Tominaga N., 2013, Annual Review of Astronomy and Astrophysics, 51, 457

Özel F., Freire P., 2016, ARA\&A, 54, 401

Perego A., Hempel M., Fröhlich C., Ebinger K., Eichler M., Casanova J., Liebendörfer M., Thielemann F. K., 2015, Astrophysical Journal, 806,275

Pignatari M., Gallino R., Heil M., Wiescher M., Käppeler F., Herwig F., Bisterzo S., 2010, ApJ, 710, 1557

Pignatari M., Göbel K., Reifarth R., Travaglio C., 2016a, International Journal of Modern Physics E, 25

Pignatari M., et al., 2016b, The Astrophysical Journal Supplement Series, 225, 24

Pignatari M., Hoppe P., Trappitsch R., Fryer C., Timmes F. X., Herwig F., Hirschi R., 2018, Geochimica Cosmochimica Acta, 221, 37

Portegies Zwart S., Pelupessy I., Van Elteren A., Wijnen T. P., Lugaro M., 2018, Astronomy and Astrophysics, 616, 1

Raiteri C. M., Busso M., Gallinó R., Picchio G., 1991, ApJ, 371, 665

Rapp W., Görres J., Wiescher M., Schatz H., Käppeler F., 2006, ApJ, 653, 474

Rauscher T., Heger A., Hoffman R. D., Woosley S. E., 2002, The Astrophysical Journal, 576, 323

Rauscher T., Dauphas N., Dillmann I., Fröhlich C., Fülöp Z., Gyürky G., 2013, Reports on Progress in Physics, 76, 066201

Ritter C., Herwig F,, Jones S., Pignatari M., Fryer C., Hirschi R., 2018, Monthly Notices of the Royal Astronomical Society, 480,538

Sieverding A., Martínez-Pinedo G., Huther L., Langanke K., Heger A., 2018, The Astrophysical Journal, 865, 143

Takahashi K., Yokoi K., 1987, Atomic Data and Nuclear Data Tables, 36, 375

Takigawa A., Miki J., Tachibana S., Huss G. R., Tominaga N., Umeda H., Nomoto K., 2008, The Astrophysical Journal, 688,1382

Thielemann F. K., Arnett W. D., 1985, The Astrophysical Journal, 295, 604

Timmes F. X., Woosley S. E., Hartmann D. H., Hoffman R. D., Weaver T. A., Matteucci F., 1995, The Astrophysical Journal, 449, 204

Travaglio C., Rauscher T., Heger A., Pignatari M., West C., 2018, The Astrophysical Journal, 854, 18

Truran J. W., Arnett W. D., 1970, The Astrophysical Journal, 160, 181

Truran J. W., Cameron A. G. W., 1978, The Astrophysical Journal, 219, 226 
Vescovi D., et al., 2018, The Astrophysical Journal, 863, 115

Wasserburg G. J., Karakas A. I., Lugaro M., 2017, The Astrophysical Journal, 836, 126

Weaver T. A., Zimmerman G. B., Woosley S. E., 1978, The Astrophysical Journal, 225, 1021

Woosley S. E., Heger A., 2007, Physics Reports, 442, 269

Woosley S. E., Hoffman R. D., 1992, The Astrophysical Journal, 395, 202

Woosley S. E., Howard W. M., 1978, The Astrophysical Journal Supplement Series, 36, 285

Woosley S. E., Weaver T. A., 1995, The Astrophysical Journal Supplement Series, 101, 181

Woosley S. E., Arnett W. D., Clayton D. D., 1973, The Astrophysical Journal Supplement Series, 26, 231

Yagüe López A., Côté B., Lugaro M., 2021, The Astrophysical Journal, 915, 128 
APPENDIX A: TABLE OF EJECTED YIELDS

This paper has been typeset from a TEX/LATEX file prepared by the author. 




Table A1. Complete table of decayed isotopic yields for all models in mass fraction. $\mathrm{M}_{\text {prog }}$ is the progenitor mass, $\mathrm{E}_{\text {exp }}$ is the explosion energy, $\mathrm{M}_{\text {rem }}$ is the remnant mass. 


\begin{tabular}{|c|c|c|c|c|c|c|c|c|}
\hline $\begin{array}{r}\mathrm{M}_{\text {prog }} \\
\quad\left(\mathrm{M}_{\odot}\right)\end{array}$ & $\begin{array}{r}\mathrm{E}_{\exp } \\
\left(\times 10^{51} \text { ergs }\right)\end{array}$ & $\begin{array}{r}\mathrm{M}_{\text {rem }} \\
\left(\mathrm{M}_{\odot}\right)\end{array}$ & ${ }^{53} \mathrm{Mn}$ & ${ }^{55} \mathrm{Mn}$ & ${ }^{60} \mathrm{Fe}$ & ${ }^{56} \mathrm{Fe}$ & ${ }^{92} \mathrm{Nb}$ & ${ }^{92} \mathrm{Mo}$ \\
\hline 15 & 1.90 & 1.62 & $6.915 \times 10^{-5}$ & $3.118 \times 10^{-4}$ & $3.426 \times 10^{-5}$ & $5.139 \times 10^{-2}$ & $5.466 \times 10^{-8}$ & $3.856 \times 10^{-6}$ \\
\hline 15 & 1.86 & 1.63 & $6.908 \times 10^{-5}$ & $3.144 \times 10^{-4}$ & $3.339 \times 10^{-5}$ & $5.140 \times 10^{-2}$ & $6.232 \times 10^{-8}$ & $3.293 \times 10^{-6}$ \\
\hline 15 & 1.94 & 1.61 & $6.937 \times 10^{-5}$ & $3.118 \times 10^{-4}$ & $3.488 \times 10^{-5}$ & $5.133 \times 10^{-2}$ & $5.133 \times 10^{-8}$ & $4.230 \times 10^{-6}$ \\
\hline 15 & 0.54 & 1.91 & $7.023 \times 10^{-7}$ & $1.183 \times 10^{-4}$ & $3.021 \times 10^{-5}$ & $1.188 \times 10^{-2}$ & $2.821 \times 10^{-12}$ & $8.406 \times 10^{-9}$ \\
\hline 15 & 0.92 & 1.75 & $5.459 \times 10^{-5}$ & $3.309 \times 10^{-4}$ & $3.318 \times 10^{-5}$ & $4.090 \times 10^{-2}$ & $1.383 \times 10^{-8}$ & $2.217 \times 10^{-6}$ \\
\hline 15 & 1.69 & 1.52 & $7.376 \times 10^{-5}$ & $3.163 \times 10^{-4}$ & $3.548 \times 10^{-5}$ & $5.069 \times 10^{-2}$ & $8.207 \times 10^{-7}$ & $8.583 \times 10^{-5}$ \\
\hline 15 & 0.34 & 1.94 & $5.264 \times 10^{-7}$ & $1.209 \times 10^{-4}$ & $3.032 \times 10^{-5}$ & $1.175 \times 10^{-2}$ & $2.819 \times 10^{-12}$ & $8.429 \times 10^{-9}$ \\
\hline 15 & 3.43 & 1.51 & $6.329 \times 10^{-5}$ & $3.158 \times 10^{-4}$ & $1.275 \times 10^{-4}$ & $5.146 \times 10^{-2}$ & $5.548 \times 10^{-7}$ & $7.983 \times 10^{-5}$ \\
\hline 15 & 2.06 & 1.59 & $7.267 \times 10^{-5}$ & $3.118 \times 10^{-4}$ & $3.625 \times 10^{-5}$ & $5.110 \times 10^{-2}$ & $3.600 \times 10^{-7}$ & $4.755 \times 10^{-5}$ \\
\hline 15 & 2.24 & 1.56 & $7.536 \times 10^{-5}$ & $3.060 \times 10^{-4}$ & $4.002 \times 10^{-5}$ & $5.087 \times 10^{-2}$ & $1.255 \times 10^{-6}$ & $1.820 \times 10^{-4}$ \\
\hline 15 & 2.60 & 1.52 & $7.369 \times 10^{-5}$ & $3.058 \times 10^{-4}$ & $5.501 \times 10^{-5}$ & $5.064 \times 10^{-2}$ & $1.351 \times 10^{-6}$ & $2.302 \times 10^{-4}$ \\
\hline 15 & 0.74 & 1.73 & $5.220 \times 10^{-5}$ & $3.358 \times 10^{-4}$ & $3.232 \times 10^{-5}$ & $4.741 \times 10^{-2}$ & $1.178 \times 10^{-8}$ & $2.420 \times 10^{-6}$ \\
\hline 15 & 0.82 & 1.88 & $6.729 \times 10^{-6}$ & $1.325 \times 10^{-4}$ & $3.043 \times 10^{-5}$ & $1.197 \times 10^{-2}$ & $2.933 \times 10^{-12}$ & $8.360 \times 10^{-9}$ \\
\hline 15 & 10.7 & 1.53 & $6.301 \times 10^{-5}$ & $3.088 \times 10^{-4}$ & $3.087 \times 10^{-5}$ & $5.236 \times 10^{-2}$ & $3.969 \times 10^{-7}$ & $4.268 \times 10^{-5}$ \\
\hline 15 & 2.47 & 1.52 & $6.653 \times 10^{-5}$ & $3.345 \times 10^{-4}$ & $3.685 \times 10^{-5}$ & $5.327 \times 10^{-2}$ & $6.125 \times 10^{-8}$ & $2.264 \times 10^{-6}$ \\
\hline 15 & 4.79 & 1.50 & $5.896 \times 10^{-5}$ & $3.184 \times 10^{-4}$ & $2.030 \times 10^{-4}$ & $5.325 \times 10^{-2}$ & $5.236 \times 10^{-7}$ & $1.051 \times 10^{-4}$ \\
\hline 15 & 2.63 & 1.53 & $6.964 \times 10^{-5}$ & $3.268 \times 10^{-4}$ & $7.601 \times 10^{-5}$ & $5.279 \times 10^{-2}$ & $5.454 \times 10^{-7}$ & $6.486 \times 10^{-5}$ \\
\hline 20 & 2.85 & 1.74 & $4.955 \times 10^{-4}$ & $6.749 \times 10^{-4}$ & $2.139 \times 10^{-5}$ & $2.600 \times 10^{-2}$ & $1.145 \times 10^{-12}$ & $9.238 \times 10^{-9}$ \\
\hline 20 & 1.47 & 2.23 & $6.496 \times 10^{-5}$ & $3.043 \times 10^{-4}$ & $2.093 \times 10^{-5}$ & $1.318 \times 10^{-2}$ & $8.751 \times 10^{-15}$ & $8.972 \times 10^{-9}$ \\
\hline 20 & 2.50 & 1.93 & $5.005 \times 10^{-6}$ & $2.319 \times 10^{-4}$ & $2.201 \times 10^{-5}$ & $1.373 \times 10^{-2}$ & $1.019 \times 10^{-12}$ & $9.226 \times 10^{-9}$ \\
\hline 20 & 5.03 & 1.74 & $4.616 \times 10^{-4}$ & $6.219 \times 10^{-4}$ & $3.025 \times 10^{-5}$ & $2.914 \times 10^{-2}$ & $4.756 \times 10^{-9}$ & $2.087 \times 10^{-7}$ \\
\hline 20 & 2.76 & 1.76 & $5.022 \times 10^{-4}$ & $6.626 \times 10^{-4}$ & $2.163 \times 10^{-5}$ & $2.443 \times 10^{-2}$ & $1.225 \times 10^{-12}$ & $9.242 \times 10^{-9}$ \\
\hline 20 & 2.60 & 1.90 & $5.537 \times 10^{-5}$ & $1.792 \times 10^{-4}$ & $2.217 \times 10^{-5}$ & $1.590 \times 10^{-2}$ & $1.222 \times 10^{-12}$ & $9.235 \times 10^{-9}$ \\
\hline 20 & 2.43 & 1.86 & $2.732 \times 10^{-4}$ & $2.909 \times 10^{-4}$ & $2.139 \times 10^{-5}$ & $1.931 \times 10^{-2}$ & $1.140 \times 10^{-12}$ & $9.245 \times 10^{-9}$ \\
\hline 20 & 1.04 & 2.47 & $6.232 \times 10^{-5}$ & $2.961 \times 10^{-4}$ & $1.840 \times 10^{-5}$ & $1.340 \times 10^{-2}$ & $6.253 \times 10^{-15}$ & $8.972 \times 10^{-9}$ \\
\hline 20 & 4.15 & 1.85 & $4.640 \times 10^{-4}$ & $6.686 \times 10^{-4}$ & $2.895 \times 10^{-5}$ & $2.362 \times 10^{-2}$ & $8.999 \times 10^{-12}$ & $9.225 \times 10^{-9}$ \\
\hline 20 & 0.65 & 3.03 & $3.575 \times 10^{-7}$ & $1.130 \times 10^{-4}$ & $1.287 \times 10^{-5}$ & $1.248 \times 10^{-2}$ & $4.025 \times 10^{-15}$ & $8.972 \times 10^{-9}$ \\
\hline 20 & 0.78 & 2.85 & $4.169 \times 10^{-5}$ & $2.234 \times 10^{-4}$ & $1.461 \times 10^{-5}$ & $1.376 \times 10^{-2}$ & $4.730 \times 10^{-15}$ & $8.972 \times 10^{-9}$ \\
\hline 20 & 0.84 & 2.62 & $5.270 \times 10^{-5}$ & $2.706 \times 10^{-4}$ & $1.687 \times 10^{-5}$ & $1.349 \times 10^{-2}$ & $5.631 \times 10^{-15}$ & $8.972 \times 10^{-9}$ \\
\hline 20 & 1.00 & 2.35 & $6.696 \times 10^{-5}$ & $3.052 \times 10^{-4}$ & $1.962 \times 10^{-5}$ & $1.328 \times 10^{-2}$ & $6.741 \times 10^{-15}$ & $8.972 \times 10^{-9}$ \\
\hline 20 & 1.65 & 1.78 & $2.798 \times 10^{-4}$ & $2.877 \times 10^{-4}$ & $2.132 \times 10^{-5}$ & $1.964 \times 10^{-2}$ & $6.240 \times 10^{-13}$ & $9.326 \times 10^{-9}$ \\
\hline 20 & 0.75 & 2.76 & $5.051 \times 10^{-5}$ & $2.495 \times 10^{-4}$ & $1.548 \times 10^{-5}$ & $1,366 \times 10^{-2}$ & $5.083 \times 10^{-15}$ & $8.972 \times 10^{-9}$ \\
\hline 20 & 0.53 & 3.40 & $6.396 \times 10^{-9}$ & $1.100 \times 10^{-4}$ & $9.311 \times 10^{-6}$ & $1.238 \times 10^{-2}$ & $2.838 \times 10^{-15}$ & $8.974 \times 10^{-9}$ \\
\hline 20 & 4.33 & 1.87 & $5.017 \times 10^{-4}$ & $5.269 \times 10^{-4}$ & $2.941 \times 10^{-5}$ & $2.205 \times 10^{-2}$ & $1.019 \times 10^{-11}$ & $9.393 \times 10^{-9}$ \\
\hline 20 & 0.81 & 2.70 & $5.512 \times 10^{-5}$ & $2.710 \times 10^{-4}$ & $1.609 \times 10^{-5}$ & $1.366 \times 10^{-2}$ & $5.324 \times 10^{-15}$ & $8.972 \times 10^{-9}$ \\
\hline 20 & 1.19 & 2.28 & $6.470 \times 10^{-5}$ & $3.022 \times 10^{-4}$ & $2.035 \times 10^{-5}$ & $1.321 \times 10^{-2}$ & $7.082 \times 10^{-15}$ & $8.972 \times 10^{-9}$ \\
\hline 20 & 1.52 & 1.97 & $1.011 \times 10^{-5}$ & $2.421 \times 10^{-4}$ & $2.132 \times 10^{-5}$ & $1.367 \times 10^{-2}$ & $6.932 \times 10^{-13}$ & $9.209 \times 10^{-9}$ \\
\hline 20 & 1.39 & 1.93 & $1.559 \times 10^{-6}$ & $1.763 \times 10^{-4}$ & $2.132 \times 10^{-5}$ & $1.393 \times 10^{-2}$ & $6.069 \times 10^{-13}$ & $9.299 \times 10^{-9}$ \\
\hline 20 & 8.86 & 1.74 & $5.526 \times 10^{-4}$ & $6.588 \times 10^{-4}$ & $1.008 \times 10^{-4}$ & $3.112 \times 10^{-2}$ & $1.858 \times 10^{-7}$ & $1.409 \times 10^{-6}$ \\
\hline 20 & 0.85 & 2.62 & $5.501 \times 10^{-5}$ & $2.776 \times 10^{-4}$ & $1.688 \times 10^{-5}$ & $1.353 \times 10^{-2}$ & $5.634 \times 10^{-15}$ & $8.972 \times 10^{-9}$ \\
\hline 25 & 1.92 & 3.13 & $7.808 \times 10^{-4}$ & $5.953 \times 10^{-4}$ & $1.274 \times 10^{-4}$ & $4.792 \times 10^{-2}$ & $6.085 \times 10^{-11}$ & $1.289 \times 10^{-8}$ \\
\hline 25 & 3.07 & 1.83 & $7.810 \times 10^{-4}$ & $5.935 \times 10^{-4}$ & $1.277 \times 10^{-4}$ & $4.748 \times 10^{-2}$ & $1.198 \times 10^{-8}$ & $1.308 \times 10^{-8}$ \\
\hline 25 & 3.30 & 2.35 & $3.633 \times 10^{-4}$ & $2.342 \times 10^{-4}$ & $1.234 \times 10^{-4}$ & $1.637 \times 10^{-2}$ & $5.987 \times 10^{-11}$ & $1.265 \times 10^{-8}$ \\
\hline 25 & 1.04 & 1.84 & $5.222 \times 10^{-4}$ & $4.567 \times 10^{-4}$ & $9.402 \times 10^{-5}$ & $4.215 \times 10^{-2}$ & $9.485 \times 10^{-9}$ & $1.539 \times 10^{-8}$ \\
\hline 25 & 9.73 & 2.35 & $4.420 \times 10^{-5}$ & $9.458 \times 10^{-5}$ & $2.069 \times 10^{-4}$ & $1.075 \times 10^{-2}$ & $6.123 \times 10^{-11}$ & $1.487 \times 10^{-8}$ \\
\hline 25 & 1.20 & 1.84 & $6.260 \times 10^{-4}$ & $4.565 \times 10^{-4}$ & $9.402 \times 10^{-5}$ & $4.215 \times 10^{-2}$ & $9.015 \times 10^{-9}$ & $1.543 \times 10^{-8}$ \\
\hline 25 & 7.42 & 2.37 & $6.758 \times 10^{-4}$ & $8.669 \times 10^{-4}$ & $2.380 \times 10^{-4}$ & $2.868 \times 10^{-2}$ & $2.667 \times 10^{-11}$ & $9.166 \times 10^{-9}$ \\
\hline 25 & 18.4 & 2.35 & $4.348 \times 10^{-5}$ & $9.310 \times 10^{-5}$ & $2.898 \times 10^{-4}$ & $1.058 \times 10^{-2}$ & $6.182 \times 10^{-11}$ & $1.492 \times 10^{-8}$ \\
\hline 25 & 14.8 & 2.35 & $6.762 \times 10^{-4}$ & $8.919 \times 10^{-4}$ & $4.381 \times 10^{-4}$ & $3.042 \times 10^{-2}$ & $1.179 \times 10^{-10}$ & $1.750 \times 10^{-8}$ \\
\hline 25 & 2.78 & 2.35 & $3.634 \times 10^{-4}$ & $2.340 \times 10^{-4}$ & $1.234 \times 10^{-4}$ & $1.637 \times 10^{-2}$ & $5.973 \times 10^{-11}$ & $1.265 \times 10^{-8}$ \\
\hline 25 & 0.89 & 4.66 & $7.694 \times 10^{-9}$ & $6.139 \times 10^{-5}$ & $5.999 \times 10^{-5}$ & $6.803 \times 10^{-3}$ & $2.556 \times 10^{-14}$ & $4.650 \times 10^{-9}$ \\
\hline 25 & 70 & 2.35 & $3.633 \times 10^{-4}$ & $2.341 \times 10^{-4}$ & $1.234 \times 10^{-4}$ & $1.637 \times 10^{-2}$ & $5.989 \times 10^{-11}$ & $1.265 \times 10^{-8}$ \\
\hline 25 & 0.92 & 1.84 & $6.260 \times 10^{-4}$ & $4.565 \times 10^{-4}$ & $9.402 \times 10^{-5}$ & $4.215 \times 10^{-2}$ & $9.015 \times 10^{-9}$ & $1.543 \times 10^{-8}$ \\
\hline 25 & 2.64 & 2.35 & $3.634 \times 10^{-4}$ & $2.340 \times 10^{-4}$ & $1.234 \times 10^{-4}$ & $1.637 \times 10^{-2}$ & $5.971 \times 10^{-11}$ & $1.265 \times 10^{-8}$ \\
\hline & 6.17 & 2.38 & $6.681 \times 10^{-4}$ & $8.691 \times 10^{-4}$ & $2.039 \times 10^{-4}$ & $2.741 \times 10^{-2}$ & $2.670 \times 10^{-11}$ & $9.156 \times 10^{-9}$ \\
\hline & 4.73 & 2.38 & $4.989 \times 10^{-4}$ & $6.807 \times 10^{-4}$ & $1.265 \times 10^{-4}$ & $3.242 \times 10^{-2}$ & $1.423 \times 10^{-10}$ & $2.099 \times 10^{-8}$ \\
\hline & 1.52 & 1.83 & $6.260 \times 10^{-4}$ & $4.565 \times 10^{-4}$ & $9.402 \times 10^{-5}$ & $4.215 \times 10^{-2}$ & $9.015 \times 10^{-9}$ & $1.543 \times 10^{-8}$ \\
\hline & 1.57 & 3.73 & $1.169 \times 10^{-8}$ & $6.298 \times 10^{-5}$ & $6.926 \times 10^{-5}$ & $6.956 \times 10^{-3}$ & $3.245 \times 10^{-14}$ & $4.638 \times 10^{-9}$ \\
\hline 25 & 0.99 & 4.89 & $6.809 \times 10^{-9}$ & $6.097 \times 10^{-5}$ & $5.979 \times 10^{-5}$ & $6.759 \times 10^{-3}$ & $2.412 \times 10^{-14}$ & $4.649 \times 10^{-9}$ \\
\hline 25 & 8.40 & 2.38 & $1.776 \times 10^{-5}$ & $8.317 \times 10^{-5}$ & $2.131 \times 10^{-4}$ & $9.851 \times 10^{-3}$ & $6.121 \times 10^{-11}$ & $1.485 \times 10^{-8}$ \\
\hline 25 & 2.53 & 2.35 & $3.634 \times 10^{-4}$ & $2.340 \times 10^{-4}$ & $1.234 \times 10^{-4}$ & $1.637 \times 10^{-2}$ & $5.968 \times 10^{-11}$ & $1.265 \times 10^{-8}$ \\
\hline 25 & 4.72 & 2.35 & $3.633 \times 10^{-4}$ & $2.343 \times 10^{-4}$ & $1.234 \times 10^{-4}$ & $1.637 \times 10^{-2}$ & $5.989 \times 10^{-11}$ & $1.265 \times 10^{-8}$ \\
\hline
\end{tabular}

Table A2. Complete table of decayed isotopic yields for all models in mass fraction. $\mathrm{M}_{\text {prog }}$ is the progenitor mass, $\mathrm{E}_{\exp }$ is the explosion energy, $\mathrm{M}_{\text {rem }}$ is the remnant mass. 


\begin{tabular}{|c|c|c|c|c|c|c|c|c|}
\hline $\begin{array}{r}\mathrm{M}_{\text {prog }} \\
\left(\mathrm{M}_{\odot}\right)\end{array}$ & $\begin{array}{r}\mathrm{E}_{\text {exp }} \\
\left(\times 10^{51} \begin{array}{l}\text { ergs })\end{array}\right.\end{array}$ & $\begin{array}{r}\mathrm{M}_{r e m} \\
\left(\mathrm{M}_{\odot}\right)\end{array}$ & ${ }^{97} \mathrm{Tc}$ & ${ }^{98} \mathrm{Ru}$ & ${ }^{98} \mathrm{Tc}$ & ${ }^{98} \mathrm{Ru}$ & ${ }^{107} \mathrm{Pd}$ & ${ }^{108} \mathrm{Pd}$ \\
\hline 15 & 1.90 & 1.62 & $2.839 \times 10^{-11}$ & $9.794 \times 10^{-10}$ & $1.129 \times 10^{-12}$ & $9.794 \times 10^{-10}$ & $4.017 \times 10^{-10}$ & $1.116 \times 10^{-8}$ \\
\hline 15 & 1.86 & 1.63 & $2.876 \times 10^{-11}$ & $9.795 \times 10^{-10}$ & $1.123 \times 10^{-12}$ & $9.795 \times 10^{-10}$ & $4.022 \times 10^{-10}$ & $1.119 \times 10^{-8}$ \\
\hline 15 & 1.94 & 1.61 & $2.861 \times 10^{-11}$ & $9.808 \times 10^{-10}$ & $1.133 \times 10^{-12}$ & $9.808 \times 10^{-10}$ & $4.015 \times 10^{-10}$ & $1.116 \times 10^{-8}$ \\
\hline 15 & 0.54 & 1.91 & $2.881 \times 10^{-11}$ & $9.627 \times 10^{-10}$ & $1.255 \times 10^{-12}$ & $9.627 \times 10^{-10}$ & $4.516 \times 10^{-10}$ & $1.150 \times 10^{-8}$ \\
\hline 15 & 0.92 & 1.75 & $2.864 \times 10^{-11}$ & $9.842 \times 10^{-10}$ & $1.067 \times 10^{-12}$ & $9.842 \times 10^{-10}$ & $3.981 \times 10^{-10}$ & $1.120 \times 10^{-8}$ \\
\hline 15 & 1.69 & 1.52 & $9.054 \times 10^{-11}$ & $1.201 \times 10^{-9}$ & $1.158 \times 10^{-12}$ & $1.201 \times 10^{-9}$ & $3.983 \times 10^{-10}$ & $1.117 \times 10^{-8}$ \\
\hline 15 & 0.34 & 1.94 & $2.925 \times 10^{-11}$ & $9.576 \times 10^{-10}$ & $1.192 \times 10^{-12}$ & $9.576 \times 10^{-10}$ & $4.534 \times 10^{-10}$ & $1.152 \times 10^{-8}$ \\
\hline 15 & 3.43 & 1.51 & $6.779 \times 10^{-11}$ & $1.164 \times 10^{-9}$ & $8.463 \times 10^{-13}$ & $1.164 \times 10^{-9}$ & $4.342 \times 10^{-10}$ & $1.093 \times 10^{-8}$ \\
\hline 15 & 2.06 & 1.59 & $6.222 \times 10^{-11}$ & $1.060 \times 10^{-9}$ & $1.150 \times 10^{-12}$ & $1.060 \times 10^{-9}$ & $4.003 \times 10^{-10}$ & $1.115 \times 10^{-8}$ \\
\hline 15 & 2.24 & 1.56 & $1.495 \times 10^{-10}$ & $1.294 \times 10^{-9}$ & $1.183 \times 10^{-12}$ & $1.294 \times 10^{-9}$ & $3.960 \times 10^{-10}$ & $1.115 \times 10^{-8}$ \\
\hline 15 & 2.60 & 1.52 & $1.545 \times 10^{-10}$ & $1.345 \times 10^{-9}$ & $1.123 \times 10^{-12}$ & $1.345 \times 10^{-9}$ & $3.834 \times 10^{-10}$ & $1.113 \times 10^{-8}$ \\
\hline 15 & 0.74 & 1.73 & $2.959 \times 10^{-11}$ & $9.900 \times 10^{-10}$ & $1.038 \times 10^{-12}$ & $9.900 \times 10^{-10}$ & $4.031 \times 10^{-10}$ & $1.124 \times 10^{-8}$ \\
\hline 15 & 0.82 & 1.88 & $2.789 \times 10^{-11}$ & $9.652 \times 10^{-10}$ & $1.140 \times 10^{-12}$ & $9.652 \times 10^{-10}$ & $4.414 \times 10^{-10}$ & $1.146 \times 10^{-8}$ \\
\hline 15 & 10.7 & 1.53 & $5.590 \times 10^{-11}$ & $1.176 \times 10^{-9}$ & $1.093 \times 10^{-12}$ & $1.176 \times 10^{-9}$ & $3.856 \times 10^{-10}$ & $1.096 \times 10^{-8}$ \\
\hline 15 & 2.47 & 1.52 & $2.837 \times 10^{-11}$ & $1.006 \times 10^{-9}$ & $1.122 \times 10^{-12}$ & $1.006 \times 10^{-9}$ & $3.944 \times 10^{-10}$ & $1.106 \times 10^{-8}$ \\
\hline 15 & 4.79 & 1.50 & $7.164 \times 10^{-11}$ & $1.239 \times 10^{-9}$ & $3.760 \times 10^{-13}$ & $1.239 \times 10^{-9}$ & $4.935 \times 10^{-10}$ & $1.081 \times 10^{-8}$ \\
\hline 15 & 2.63 & 1.53 & $6.674 \times 10^{-11}$ & $1.192 \times 10^{-9}$ & $1.032 \times 10^{-12}$ & $1.192 \times 10^{-9}$ & $3.814 \times 10^{-10}$ & $1.092 \times 10^{-8}$ \\
\hline 20 & 2.85 & 1.74 & $3.259 \times 10^{-11}$ & $9.824 \times 10^{-10}$ & $3.968 \times 10^{-12}$ & $9.824 \times 10^{-10}$ & $1.300 \times 10^{-9}$ & $1.659 \times 10^{-8}$ \\
\hline 20 & 1.47 & 2.23 & $3.088 \times 10^{-11}$ & $9.184 \times 10^{-10}$ & $1.848 \times 10^{-12}$ & $9.184 \times 10^{-10}$ & $1.278 \times 10^{-9}$ & $1.636 \times 10^{-8}$ \\
\hline 20 & 2.50 & 1.93 & $3.336 \times 10^{-11}$ & $9.793 \times 10^{-10}$ & $4.012 \times 10^{-12}$ & $9.793 \times 10^{-10}$ & $1.287 \times 10^{-9}$ & $1.674 \times 10^{-8}$ \\
\hline 20 & 5.03 & 1.74 & $5.724 \times 10^{-11}$ & $1.072 \times 10^{-9}$ & $4.115 \times 10^{-12}$ & $1.072 \times 10^{-9}$ & $1.188 \times 10^{-9}$ & $1.629 \times 10^{-8}$ \\
\hline 20 & 2.76 & 1.76 & $3.286 \times 10^{-11}$ & $9.836 \times 10^{-10}$ & $3.965 \times 10^{-12}$ & $9.836 \times 10^{-10}$ & $1.295 \times 10^{-9}$ & $1.658 \times 10^{-8}$ \\
\hline 20 & 2.60 & 1.90 & $3.282 \times 10^{-11}$ & $9.862 \times 10^{-10}$ & $3.956 \times 10^{-12}$ & $9.862 \times 10^{-10}$ & $1.290 \times 10^{-9}$ & $1.656 \times 10^{-8}$ \\
\hline 20 & 2.43 & 1.86 & $3.322 \times 10^{-11}$ & $9.808 \times 10^{-10}$ & $3.692 \times 10^{-12}$ & $9.808 \times 10^{-10}$ & $1.300 \times 10^{-9}$ & $1.658 \times 10^{-8}$ \\
\hline 20 & 1.04 & 2.47 & $3.085 \times 10^{-11}$ & $9.184 \times 10^{-10}$ & $1.886 \times 10^{-12}$ & $9.184 \times 10^{-10}$ & $1.171 \times 10^{-9}$ & $1.575 \times 10^{-8}$ \\
\hline 20 & 4.15 & 1.85 & $5.807 \times 10^{-11}$ & $1.071 \times 10^{-9}$ & $4.459 \times 10^{-12}$ & $1.071 \times 10^{-9}$ & $1.219 \times 10^{-9}$ & $1.639 \times 10^{-8}$ \\
\hline 20 & 0.65 & 3.03 & $3.088 \times 10^{-11}$ & $9.186 \times 10^{-10}$ & $1.850 \times 10^{-12}$ & $9.186 \times 10^{-10}$ & $9.223 \times 10^{-10}$ & $1.434 \times 10^{-8}$ \\
\hline 20 & 0.78 & 2.85 & $3.088 \times 10^{-11}$ & $9.185 \times 10^{-10}$ & $1.854 \times 10^{-12}$ & $9.185 \times 10^{-10}$ & $1.002 \times 10^{-9}$ & $1.479 \times 10^{-8}$ \\
\hline 20 & 0.84 & 2.62 & $3.087 \times 10^{-11}$ & $9.185 \times 10^{-10}$ & $1.855 \times 10^{-12}$ & $9.185 \times 10^{-10}$ & $1.104 \times 10^{-9}$ & $1.537 \times 10^{-8}$ \\
\hline 20 & 1.00 & 2.35 & $3.086 \times 10^{-11}$ & $9.184 \times 10^{-10}$ & $1.869 \times 10^{-12}$ & $9.184 \times 10^{-10}$ & $1.225 \times 10^{-9}$ & $1.605 \times 10^{-8}$ \\
\hline 20 & 1.65 & 1.78 & $3.373 \times 10^{-11}$ & $9.790 \times 10^{-10}$ & $2.174 \times 10^{-12}$ & $9.790 \times 10^{-10}$ & $1.306 \times 10^{-9}$ & $1.654 \times 10^{-8}$ \\
\hline 20 & 0.75 & 2.76 & $3.088 \times 10^{-11}$ & $9.185 \times 10^{-10}$ & $1.852 \times 10^{-12}$ & $9,185 \times 10^{-10}$ & $1.042 \times 10^{-9}$ & $1.502 \times 10^{-8}$ \\
\hline 20 & 0.53 & 3.40 & $3.091 \times 10^{-11}$ & $9.190 \times 10^{-10}$ & $1.849 \times 10^{-12}$ & $9.190 \times 10^{-10}$ & $7.578 \times 10^{-10}$ & $1.342 \times 10^{-8}$ \\
\hline 20 & 4.33 & 1.87 & $6.347 \times 10^{-11}$ & $1.192 \times 10^{-9}$ & 4. $429 \times 10^{-12}$ & $1.192 \times 10^{-9}$ & $1.198 \times 10^{-9}$ & $1.630 \times 10^{-8}$ \\
\hline 20 & 0.81 & 2.70 & $3.087 \times 10^{-11}$ & $9.185 \times 10^{-10}$ & $1.857 \times 10^{-12}$ & $9.185 \times 10^{-10}$ & $1.069 \times 10^{-9}$ & $1.517 \times 10^{-8}$ \\
\hline 20 & 1.19 & 2.28 & $3.082 \times 10^{-11}$ & $9.184 \times 10^{-10}$ & $1.928 \times 10^{-12}$ & $9.184 \times 10^{-10}$ & $1.256 \times 10^{-9}$ & $1.622 \times 10^{-8}$ \\
\hline 20 & 1.52 & 1.97 & $3.385 \times 10^{-11}$ & $9.654 \times 10^{-10}$ & $2.187 \times 10^{-12}$ & $9.654 \times 10^{-10}$ & $1.305 \times 10^{-9}$ & $1.654 \times 10^{-8}$ \\
\hline 20 & 1.39 & 1.93 & $3.370 \times 10^{-11}$ & $9.759 \times 10^{-10}$ & $2.072 \times 10^{-12}$ & $9.759 \times 10^{-10}$ & $1.306 \times 10^{-9}$ & $1.654 \times 10^{-8}$ \\
\hline 20 & 8.86 & 1.74 & $7.480 \times 10^{-11}$ & $1.467 \times 10^{-9}$ & $4.075 \times 10^{-12}$ & $1.467 \times 10^{-9}$ & $1.022 \times 10^{-9}$ & $1.519 \times 10^{-8}$ \\
\hline 20 & 0.85 & 2.62 & $3.087 \times 10^{-11}$ & $9.185 \times 10^{-10}$ & $1.859 \times 10^{-12}$ & $9.185 \times 10^{-10}$ & $1.105 \times 10^{-9}$ & $1.537 \times 10^{-8}$ \\
\hline 25 & 1.92 & 3.13 & $2.352 \times 10^{-10}$ & $3.175 \times 10^{-9}$ & $8.409 \times 10^{-12}$ & $3.175 \times 10^{-9}$ & $1.666 \times 10^{-9}$ & $1.608 \times 10^{-8}$ \\
\hline 25 & 3.07 & 1.83 & $2.362 \times 10^{-10}$ & $3.175 \times 10^{-9}$ & $8.548 \times 10^{-12}$ & $3.175 \times 10^{-9}$ & $8.621 \times 10^{-6}$ & $3.193 \times 10^{-5}$ \\
\hline 25 & 3.30 & 2.35 & $2.325 \times 10^{-10}$ & $3.157 \times 10^{-9}$ & $8.548 \times 10^{-12}$ & $3.157 \times 10^{-9}$ & $1.676 \times 10^{-9}$ & $1.615 \times 10^{-8}$ \\
\hline 25 & 1.04 & 1.84 & $1.609 \times 10^{-10}$ & $1.925 \times 10^{-9}$ & $7.192 \times 10^{-12}$ & $1.925 \times 10^{-9}$ & $1.995 \times 10^{-9}$ & $1.757 \times 10^{-8}$ \\
\hline 25 & 9.73 & 2.35 & $2.316 \times 10^{-10}$ & $3.007 \times 10^{-9}$ & $6.995 \times 10^{-12}$ & $3.007 \times 10^{-9}$ & $1.525 \times 10^{-9}$ & $1.517 \times 10^{-8}$ \\
\hline 25 & 1.20 & 1.84 & $1.609 \times 10^{-10}$ & $1.925 \times 10^{-9}$ & $7.192 \times 10^{-12}$ & $1.925 \times 10^{-9}$ & $2.058 \times 10^{-9}$ & $1.758 \times 10^{-8}$ \\
\hline 25 & 7.42 & 2.37 & $1.018 \times 10^{-10}$ & $1.569 \times 10^{-9}$ & $5.071 \times 10^{-12}$ & $1.569 \times 10^{-9}$ & $1.499 \times 10^{-9}$ & $1.470 \times 10^{-8}$ \\
\hline 25 & 18.4 & 2.35 & $2.316 \times 10^{-10}$ & $3.046 \times 10^{-9}$ & $6.502 \times 10^{-12}$ & $3.046 \times 10^{-9}$ & $1.530 \times 10^{-9}$ & $1.496 \times 10^{-8}$ \\
\hline 25 & 14.8 & 2.35 & $2.787 \times 10^{-10}$ & $4.974 \times 10^{-9}$ & $6.623 \times 10^{-12}$ & $4.974 \times 10^{-9}$ & $1.210 \times 10^{-9}$ & $1.221 \times 10^{-8}$ \\
\hline 25 & 2.78 & 2.35 & $2.325 \times 10^{-10}$ & $3.157 \times 10^{-9}$ & $8.551 \times 10^{-12}$ & $3.157 \times 10^{-9}$ & $1.697 \times 10^{-9}$ & $1.617 \times 10^{-8}$ \\
\hline 25 & 0.89 & 4.66 & $3.514 \times 10^{-11}$ & $4.980 \times 10^{-10}$ & $5.453 \times 10^{-12}$ & $4.980 \times 10^{-10}$ & $1.413 \times 10^{-9}$ & $1.267 \times 10^{-8}$ \\
\hline 25 & & 2.35 & $2.325 \times 10^{-10}$ & $3.157 \times 10^{-9}$ & $8.541 \times 10^{-12}$ & $3.157 \times 10^{-9}$ & $1.669 \times 10^{-9}$ & $1.613 \times 10^{-8}$ \\
\hline 25 & 0.92 & 1.84 & $1.609 \times 10^{-10}$ & $1.925 \times 10^{-9}$ & $7.192 \times 10^{-12}$ & $1.925 \times 10^{-9}$ & $2.068 \times 10^{-9}$ & $1.758 \times 10^{-8}$ \\
\hline 25 & & 2.35 & $2.325 \times 10^{-10}$ & $3.157 \times 10^{-9}$ & $8.551 \times 10^{-12}$ & $3.157 \times 10^{-9}$ & $1.695 \times 10^{-9}$ & $1.617 \times 10^{-8}$ \\
\hline & & 2.38 & $1.018 \times 10^{-10}$ & $1.555 \times 10^{-9}$ & $5.235 \times 10^{-12}$ & $1.555 \times 10^{-9}$ & $1.519 \times 10^{-9}$ & $1.495 \times 10^{-8}$ \\
\hline & & 2.38 & $9.483 \times 10^{-11}$ & $1.496 \times 10^{-9}$ & $5.849 \times 10^{-12}$ & $1.496 \times 10^{-9}$ & $1.859 \times 10^{-9}$ & $1.675 \times 10^{-8}$ \\
\hline & 1.52 & 1.83 & $1.609 \times 10^{-10}$ & $1.925 \times 10^{-9}$ & $7.192 \times 10^{-12}$ & $1.925 \times 10^{-9}$ & $2.058 \times 10^{-9}$ & $1.758 \times 10^{-8}$ \\
\hline & 1.57 & 3.73 & $3.235 \times 10^{-11}$ & $4.964 \times 10^{-10}$ & $6.448 \times 10^{-12}$ & $4.964 \times 10^{-10}$ & $1.889 \times 10^{-9}$ & $1.560 \times 10^{-8}$ \\
\hline 25 & 0.99 & 4.89 & $3.481 \times 10^{-11}$ & $4.979 \times 10^{-10}$ & $5.792 \times 10^{-12}$ & $4.979 \times 10^{-10}$ & $1.284 \times 10^{-9}$ & $1.186 \times 10^{-8}$ \\
\hline 20 & 8.40 & 2.38 & $2.305 \times 10^{-10}$ & $3.004 \times 10^{-9}$ & $6.866 \times 10^{-12}$ & $3.004 \times 10^{-9}$ & $1.530 \times 10^{-9}$ & $1.518 \times 10^{-8}$ \\
\hline 25 & 2.53 & 2.35 & $2.325 \times 10^{-10}$ & $3.157 \times 10^{-9}$ & $8.551 \times 10^{-12}$ & $3.157 \times 10^{-9}$ & $1.694 \times 10^{-9}$ & $1.618 \times 10^{-8}$ \\
\hline 25 & 4.72 & 2.35 & $2.326 \times 10^{-10}$ & $3.157 \times 10^{-9}$ & $8.546 \times 10^{-12}$ & $3.157 \times 10^{-9}$ & $1.670 \times 10^{-9}$ & $1.614 \times 10^{-8}$ \\
\hline
\end{tabular}

Table A3. Complete table of decayed isotopic yields for all models in mass fraction. $\mathrm{M}_{\text {prog }}$ is the progenitor mass, $\mathrm{E}_{\exp }$ is the explosion energy, $\mathrm{M}_{\text {rem }}$ is the remnant mass. 


\begin{tabular}{|c|c|c|c|c|c|c|c|c|}
\hline $\begin{array}{r}\mathrm{M}_{\text {prog }} \\
\left(\mathrm{M}_{\odot}\right)\end{array}$ & $\begin{array}{r}\mathrm{E}_{\text {exp }} \\
\left(\times 10^{51} \begin{array}{l}\text { ergs })\end{array}\right.\end{array}$ & $\begin{array}{r}\mathrm{M}_{\text {rem }} \\
\left(\mathrm{M}_{\odot}\right)\end{array}$ & ${ }^{126} \mathrm{Sn}$ & ${ }^{124} \mathrm{Sn}$ & ${ }^{129} \mathrm{I}$ & ${ }^{127} \mathrm{I}$ & ${ }^{135} \mathrm{Cs}$ & ${ }^{133} \mathrm{Cs}$ \\
\hline 15 & 1.90 & 1.62 & $2.494 \times 10^{-11}$ & $6.873 \times 10^{-9}$ & $4.344 \times 10^{-10}$ & $1.138 \times 10^{-8}$ & $7.688 \times 10^{-10}$ & $1.172 \times 10^{-8}$ \\
\hline 15 & 1.86 & 1.63 & $1.900 \times 10^{-11}$ & $6.839 \times 10^{-9}$ & $4.205 \times 10^{-10}$ & $1.139 \times 10^{-8}$ & $7.504 \times 10^{-10}$ & $1.173 \times 10^{-8}$ \\
\hline 15 & 1.94 & 1.61 & $2.873 \times 10^{-11}$ & $6.897 \times 10^{-9}$ & $4.400 \times 10^{-10}$ & $1.137 \times 10^{-8}$ & $7.806 \times 10^{-10}$ & $1.171 \times 10^{-8}$ \\
\hline 15 & 0.54 & 1.91 & $2.260 \times 10^{-12}$ & $6.734 \times 10^{-9}$ & $3.054 \times 10^{-10}$ & $1.146 \times 10^{-8}$ & $7.522 \times 10^{-10}$ & $1.167 \times 10^{-8}$ \\
\hline 15 & 0.92 & 1.75 & $1.186 \times 10^{-11}$ & $6.824 \times 10^{-9}$ & $3.913 \times 10^{-10}$ & $1.142 \times 10^{-8}$ & $7.315 \times 10^{-10}$ & $1.176 \times 10^{-8}$ \\
\hline 15 & 1.69 & 1.52 & $2.773 \times 10^{-11}$ & $6.901 \times 10^{-9}$ & $4.325 \times 10^{-10}$ & $1.136 \times 10^{-8}$ & $7.726 \times 10^{-10}$ & $1.170 \times 10^{-8}$ \\
\hline 15 & 0.34 & 1.94 & $2.139 \times 10^{-12}$ & $6.736 \times 10^{-9}$ & $2.824 \times 10^{-10}$ & $1.147 \times 10^{-8}$ & $7.342 \times 10^{-10}$ & $1.168 \times 10^{-8}$ \\
\hline 15 & 3.43 & 1.51 & $8.130 \times 10^{-10}$ & $8.856 \times 10^{-9}$ & $3.737 \times 10^{-10}$ & $1.133 \times 10^{-8}$ & $9.662 \times 10^{-10}$ & $1.164 \times 10^{-8}$ \\
\hline 15 & 2.06 & 1.59 & $3.811 \times 10^{-11}$ & $6.953 \times 10^{-9}$ & $4.492 \times 10^{-10}$ & $1.135 \times 10^{-8}$ & $8.110 \times 10^{-10}$ & $1.169 \times 10^{-8}$ \\
\hline 15 & 2.24 & 1.56 & $6.181 \times 10^{-11}$ & $7.106 \times 10^{-9}$ & $4.529 \times 10^{-10}$ & $1.132 \times 10^{-8}$ & $8.787 \times 10^{-10}$ & $1.163 \times 10^{-8}$ \\
\hline 15 & 2.60 & 1.52 & $1.613 \times 10^{-10}$ & $7.655 \times 10^{-9}$ & $4.176 \times 10^{-10}$ & $1.130 \times 10^{-8}$ & $1.011 \times 10^{-9}$ & $1.154 \times 10^{-8}$ \\
\hline 15 & 0.74 & 1.73 & $8.060 \times 10^{-12}$ & $6.799 \times 10^{-9}$ & $3.617 \times 10^{-10}$ & $1.143 \times 10^{-8}$ & $7.220 \times 10^{-10}$ & $1.173 \times 10^{-8}$ \\
\hline 15 & 0.82 & 1.88 & $2.873 \times 10^{-12}$ & $6.737 \times 10^{-9}$ & $3.352 \times 10^{-10}$ & $1.145 \times 10^{-8}$ & $7.661 \times 10^{-10}$ & $1.168 \times 10^{-8}$ \\
\hline 15 & 10.7 & 1.53 & $1.790 \times 10^{-11}$ & $6.824 \times 10^{-9}$ & $3.751 \times 10^{-10}$ & $1.137 \times 10^{-8}$ & $6.664 \times 10^{-10}$ & $1.167 \times 10^{-8}$ \\
\hline 15 & 2.47 & 1.52 & $4.953 \times 10^{-11}$ & $7.016 \times 10^{-9}$ & $4.471 \times 10^{-10}$ & $1.134 \times 10^{-8}$ & $8.315 \times 10^{-10}$ & $1.167 \times 10^{-8}$ \\
\hline 15 & 4.79 & 1.50 & $1.700 \times 10^{-9}$ & $9.111 \times 10^{-9}$ & $4.826 \times 10^{-10}$ & $1.150 \times 10^{-8}$ & $8.658 \times 10^{-10}$ & $1.173 \times 10^{-8}$ \\
\hline 15 & 2.63 & 1.53 & $3.496 \times 10^{-10}$ & $8.200 \times 10^{-9}$ & $3.703 \times 10^{-10}$ & $1.129 \times 10^{-8}$ & $1.000 \times 10^{-9}$ & $1.154 \times 10^{-8}$ \\
\hline 20 & 2.85 & 1.74 & $3.038 \times 10^{-13}$ & $7.555 \times 10^{-9}$ & $4.829 \times 10^{-10}$ & $1.384 \times 10^{-8}$ & $1.093 \times 10^{-9}$ & $1,416 \times 10^{-8}$ \\
\hline 20 & 1.47 & 2.23 & $2.412 \times 10^{-13}$ & $7.549 \times 10^{-9}$ & $4.221 \times 10^{-10}$ & $1.392 \times 10^{-8}$ & $1.028 \times 10^{-9}$ & $1.427 \times 10^{-8}$ \\
\hline 20 & 2.50 & 1.93 & $3.940 \times 10^{-13}$ & $7.565 \times 10^{-9}$ & $5.158 \times 10^{-10}$ & $1.378 \times 10^{-8}$ & $1.117 \times 10^{-9}$ & $1.413 \times 10^{-8}$ \\
\hline 20 & 5.03 & 1.74 & $1.822 \times 10^{-11}$ & $7.849 \times 10^{-9}$ & $6.289 \times 10^{-10}$ & $1.357 \times 10^{-8}$ & $1.251 \times 10^{-9}$ & $1.393 \times 10^{-8}$ \\
\hline 20 & 2.76 & 1.76 & $3.481 \times 10^{-13}$ & $7.561 \times 10^{-9}$ & $4.801 \times 10^{-10}$ & $1.384 \times 10^{-8}$ & $1.088 \times 10^{-9}$ & $1.416 \times 10^{-8}$ \\
\hline 20 & 2.60 & 1.90 & $5.173 \times 10^{-13}$ & $7.574 \times 10^{-9}$ & $4.789 \times 10^{-10}$ & $1.384 \times 10^{-8}$ & $1.084 \times 10^{-9}$ & $1.416 \times 10^{-8}$ \\
\hline 20 & 2.43 & 1.86 & $2.850 \times 10^{-13}$ & $7.556 \times 10^{-9}$ & $4.679 \times 10^{-10}$ & $1.387 \times 10^{-8}$ & $1.080 \times 10^{-9}$ & $1.419 \times 10^{-8}$ \\
\hline 20 & 1.04 & 2.47 & $2.090 \times 10^{-13}$ & $7.524 \times 10^{-9}$ & $3.680 \times 10^{-10}$ & $1.380 \times 10^{-8}$ & $9.136 \times 10^{-10}$ & $1.413 \times 10^{-8}$ \\
\hline 20 & 4.15 & 1.85 & $1.116 \times 10^{-11}$ & $7.813 \times 10^{-9}$ & $6.135 \times 10^{-10}$ & $1.361 \times 10^{-8}$ & $1.236 \times 10^{-9}$ & $1.395 \times 10^{-8}$ \\
\hline 20 & 0.65 & 3.03 & $1.366 \times 10^{-13}$ & $7.467 \times 10^{-9}$ & $2.430 \times 10^{-10}$ & $1.349 \times 10^{-8}$ & $6.485 \times 10^{-10}$ & $1.378 \times 10^{-8}$ \\
\hline 20 & 0.78 & 2.85 & $1.598 \times 10^{-13}$ & $7.486 \times 10^{-9}$ & $2.831 \times 10^{-10}$ & $1.359 \times 10^{-8}$ & $7.337 \times 10^{-10}$ & $1.390 \times 10^{-8}$ \\
\hline 20 & 0.84 & 2.62 & $1.895 \times 10^{-13}$ & $7.509 \times 10^{-9}$ & $3.343 \times 10^{-10}$ & $1.372 \times 10^{-8}$ & $8.422 \times 10^{-10}$ & $1.404 \times 10^{-8}$ \\
\hline 20 & 1.00 & 2.35 & $2.245 \times 10^{-13}$ & $7.537 \times 10^{-9}$ & $3.947 \times 10^{-10}$ & 1. $387 \times 10^{-8}$ & $9.704 \times 10^{-10}$ & $1.420 \times 10^{-8}$ \\
\hline 20 & 1.65 & 1.78 & $2.480 \times 10^{-13}$ & $7.556 \times 10^{-9}$ & $4.387 \times 10^{-40}$ & $1.395 \times 10^{-8}$ & $1.061 \times 10^{-9}$ & $1.427 \times 10^{-8}$ \\
\hline 20 & 0.75 & 2.76 & $1.714 \times 10^{-13}$ & $7.495 \times 10^{-9}$ & $3.032 \times 10^{-10}$ & $1.364 \times 10^{-8}$ & $7.762 \times 10^{-10}$ & $1.395 \times 10^{-8}$ \\
\hline 20 & 0.53 & 3.40 & $8.880 \times 10^{-14}$ & $7.430 \times 10^{-9}$ & $1.604 \times 10^{-10}$ & $1.328 \times 10^{-8}$ & $4.729 \times 10^{-10}$ & $1.353 \times 10^{-8}$ \\
\hline 20 & 4.33 & 1.87 & $1.394 \times 10^{-11}$ & $7.834 \times 10^{-9}$ & $6.182 \times 10^{-10}$ & $1.358 \times 10^{-8}$ & $1.238 \times 10^{-9}$ & $1.393 \times 10^{-8}$ \\
\hline 20 & 0.81 & 2.70 & $1.794 \times 10^{-13}$ & $7.501 \times 10^{-9}$ & $3.168 \times 10^{-10}$ & $1.368 \times 10^{-8}$ & $8.052 \times 10^{-10}$ & $1.399 \times 10^{-8}$ \\
\hline 20 & 1.19 & 2.28 & $2.336 \times 10^{-13}$ & $7.544 \times 10^{-9}$ & $4.104 \times 10^{-10}$ & $1.390 \times 10^{-8}$ & $1.004 \times 10^{-9}$ & $1.424 \times 10^{-8}$ \\
\hline 20 & 1.52 & 1.97 & $2.479 \times 10^{-13}$ & $7.555 \times 10^{-9}$ & $4.384 \times 10^{-10}$ & $1.396 \times 10^{-8}$ & $1.061 \times 10^{-9}$ & $1.429 \times 10^{-8}$ \\
\hline 20 & 1.39 & 1.93 & $2.479 \times 10^{-13}$ & $7.556 \times 10^{-9}$ & $4.382 \times 10^{-10}$ & $1.396 \times 10^{-8}$ & $1.060 \times 10^{-9}$ & $1.429 \times 10^{-8}$ \\
\hline 20 & 8.86 & 1.74 & $4.995 \times 10^{-10}$ & $9.871 \times 10^{-9}$ & $6.549 \times 10^{-10}$ & $1.327 \times 10^{-8}$ & $1.394 \times 10^{-9}$ & $1.363 \times 10^{-8}$ \\
\hline 20 & 0.85 & 2.62 & $1.896 \times 10^{-13}$ & $7.509 \times 10^{-9}$ & $3.344 \times 10^{-10}$ & $1.372 \times 10^{-8}$ & $8.426 \times 10^{-10}$ & $1.404 \times 10^{-8}$ \\
\hline 25 & 1.92 & 3.13 & $1.399 \times 10^{-10}$ & $7.171 \times 10^{-9}$ & $6.040 \times 10^{-10}$ & $7.715 \times 10^{-9}$ & $1.397 \times 10^{-9}$ & $8.671 \times 10^{-9}$ \\
\hline 25 & 3.07 & 1.83 & $5.669 \times 10^{-6}$ & $3.943 \times 10^{-6}$ & $3.172 \times 10^{-6}$ & $5.095 \times 10^{-7}$ & $1.396 \times 10^{-9}$ & $5.909 \times 10^{-8}$ \\
\hline 25 & 3.30 & 2.35 & $1.119 \times 10^{-10}$ & $6.919 \times 10^{-9}$ & $6.014 \times 10^{-10}$ & $7.779 \times 10^{-9}$ & $1.408 \times 10^{-9}$ & $8.760 \times 10^{-9}$ \\
\hline 25 & 1.04 & 1.84 & $2.468 \times 10^{-11}$ & $5.372 \times 10^{-9}$ & $5.244 \times 10^{-10}$ & $8.311 \times 10^{-9}$ & $1.429 \times 10^{-9}$ & $9.398 \times 10^{-9}$ \\
\hline 25 & 9.73 & 2.35 & $1.051 \times 10^{-9}$ & $1.083 \times 10^{-8}$ & $5.732 \times 10^{-10}$ & $7.447 \times 10^{-9}$ & $1.541 \times 10^{-9}$ & $8.392 \times 10^{-9}$ \\
\hline 25 & 1.20 & 1.84 & $2.468 \times 10^{-11}$ & $5.372 \times 10^{-9}$ & $5.434 \times 10^{-10}$ & $8.317 \times 10^{-9}$ & $1.433 \times 10^{-9}$ & $9.399 \times 10^{-9}$ \\
\hline 25 & 7.42 & 2.37 & $1.251 \times 10^{-9}$ & $1.184 \times 10^{-8}$ & $6.158 \times 10^{-10}$ & $7.296 \times 10^{-9}$ & $1.564 \times 10^{-9}$ & $8.152 \times 10^{-9}$ \\
\hline 25 & 18.4 & 2.35 & $1.823 \times 10^{-9}$ & $1.221 \times 10^{-8}$ & $5.800 \times 10^{-10}$ & $7.403 \times 10^{-9}$ & $1.557 \times 10^{-9}$ & $8.333 \times 10^{-9}$ \\
\hline 25 & 14.8 & 2.35 & $4.324 \times 10^{-9}$ & $1.626 \times 10^{-8}$ & $6.155 \times 10^{-10}$ & $6.891 \times 10^{-9}$ & $1.288 \times 10^{-9}$ & $7.429 \times 10^{-9}$ \\
\hline 25 & 2.78 & 2.35 & $1.119 \times 10^{-10}$ & $6.919 \times 10^{-9}$ & $6.073 \times 10^{-10}$ & $7.779 \times 10^{-9}$ & $1.409 \times 10^{-9}$ & $8.760 \times 10^{-9}$ \\
\hline 25 & 0.89 & 4.66 & $1.290 \times 10^{-12}$ & $4.151 \times 10^{-9}$ & $3.290 \times 10^{-10}$ & $7.688 \times 10^{-9}$ & $9.497 \times 10^{-10}$ & $8.545 \times 10^{-9}$ \\
\hline 25 & & 2.35 & $1.119 \times 10^{-10}$ & $6.919 \times 10^{-9}$ & $5.942 \times 10^{-10}$ & $7.778 \times 10^{-9}$ & $1.406 \times 10^{-9}$ & $8.760 \times 10^{-9}$ \\
\hline 25 & 0.92 & 1.84 & $2.468 \times 10^{-11}$ & $5.372 \times 10^{-9}$ & $5.472 \times 10^{-10}$ & $8.318 \times 10^{-9}$ & $1.434 \times 10^{-9}$ & $9.399 \times 10^{-9}$ \\
\hline 25 & 2.64 & 2.35 & $1.119 \times 10^{-10}$ & $6.919 \times 10^{-9}$ & $6.073 \times 10^{-10}$ & $7.779 \times 10^{-9}$ & $1.409 \times 10^{-9}$ & $8.760 \times 10^{-9}$ \\
\hline & & 2.38 & $9.255 \times 10^{-10}$ & $1.086 \times 10^{-8}$ & $6.304 \times 10^{-10}$ & $7.342 \times 10^{-9}$ & $1.574 \times 10^{-9}$ & $8.219 \times 10^{-9}$ \\
\hline & & 2.38 & $3.921 \times 10^{-10}$ & $7.656 \times 10^{-9}$ & $6.826 \times 10^{-10}$ & $7.693 \times 10^{-9}$ & $1.651 \times 10^{-9}$ & $8.748 \times 10^{-9}$ \\
\hline & 1.52 & 1.83 & $2.468 \times 10^{-11}$ & $5.372 \times 10^{-9}$ & $5.434 \times 10^{-10}$ & $8.317 \times 10^{-9}$ & $1.433 \times 10^{-9}$ & $9.399 \times 10^{-9}$ \\
\hline & 1.57 & 3.73 & $8.161 \times 10^{-12}$ & $4.428 \times 10^{-9}$ & $4.637 \times 10^{-10}$ & $8.125 \times 10^{-9}$ & $1.250 \times 10^{-9}$ & $9.234 \times 10^{-9}$ \\
\hline 25 & 0.99 & 4.89 & $1.781 \times 10^{-12}$ & $4.166 \times 10^{-9}$ & $3.175 \times 10^{-10}$ & $7.512 \times 10^{-9}$ & $8.712 \times 10^{-10}$ & $8.321 \times 10^{-9}$ \\
\hline 25 & 8.40 & 2.38 & $1.109 \times 10^{-9}$ & $1.093 \times 10^{-8}$ & $5.891 \times 10^{-10}$ & $7.427 \times 10^{-9}$ & $1.561 \times 10^{-9}$ & $8.375 \times 10^{-9}$ \\
\hline 25 & 2.53 & 2.35 & $1.119 \times 10^{-10}$ & $6.919 \times 10^{-9}$ & $6.079 \times 10^{-10}$ & $7.779 \times 10^{-9}$ & $1.409 \times 10^{-9}$ & $8.760 \times 10^{-9}$ \\
\hline 25 & 4.72 & 2.35 & $1.119 \times 10^{-10}$ & $6.919 \times 10^{-9}$ & $5.981 \times 10^{-10}$ & $7.779 \times 10^{-9}$ & $1.407 \times 10^{-9}$ & $8.761 \times 10^{-9}$ \\
\hline
\end{tabular}

Table A4. Complete table of decayed isotopic yields for all models in mass fraction. $\mathrm{M}_{\text {prog }}$ is the progenitor mass, $\mathrm{E}_{\exp }$ is the explosion energy, $\mathrm{M}_{\text {rem }}$ is the remnant mass. 


\begin{tabular}{|c|c|c|c|c|c|c|c|c|}
\hline $\begin{array}{r}\mathrm{M}_{\text {prog }} \\
\left(\mathrm{M}_{\odot}\right)\end{array}$ & $\begin{array}{r}\mathrm{E}_{\text {exp }} \\
\left(\times 10^{51} \text { ergs }\right)\end{array}$ & $\begin{array}{r}\mathrm{M}_{r e m} \\
\left(\mathrm{M}_{\odot}\right)\end{array}$ & ${ }^{146} \mathrm{Sm}$ & ${ }^{144} \mathrm{Sm}$ & ${ }^{182} \mathrm{Hf}$ & ${ }^{180} \mathrm{Hf}$ & ${ }^{205} \mathrm{~Pb}$ & ${ }^{204} \mathrm{~Pb}$ \\
\hline 15 & 1.90 & 1.62 & $8.319 \times 10^{-11}$ & $1.170 \times 10^{-9}$ & $1.425 \times 10^{-10}$ & $4.693 \times 10^{-10}$ & $1.232 \times 10^{-9}$ & $1.798 \times 10^{-9}$ \\
\hline 15 & 1.86 & 1.63 & $8.308 \times 10^{-11}$ & $1.168 \times 10^{-9}$ & $1.528 \times 10^{-10}$ & $4.694 \times 10^{-10}$ & $1.254 \times 10^{-9}$ & $1.810 \times 10^{-9}$ \\
\hline 15 & 1.94 & 1.61 & $8.388 \times 10^{-11}$ & $1.173 \times 10^{-9}$ & $1.365 \times 10^{-10}$ & $4.716 \times 10^{-10}$ & $1.220 \times 10^{-9}$ & $1.790 \times 10^{-9}$ \\
\hline 15 & 0.54 & 1.91 & $8.885 \times 10^{-11}$ & $1.095 \times 10^{-9}$ & $4.533 \times 10^{-11}$ & $6.686 \times 10^{-10}$ & $1.503 \times 10^{-9}$ & $2.008 \times 10^{-9}$ \\
\hline 15 & 0.92 & 1.75 & $8.647 \times 10^{-11}$ & $1.326 \times 10^{-9}$ & $1.622 \times 10^{-10}$ & $4.758 \times 10^{-10}$ & $1.287 \times 10^{-9}$ & $1.947 \times 10^{-9}$ \\
\hline 15 & 1.69 & 1.52 & $7.681 \times 10^{-11}$ & $1.159 \times 10^{-9}$ & $1.337 \times 10^{-10}$ & $4.673 \times 10^{-10}$ & $1.227 \times 10^{-9}$ & $1.829 \times 10^{-9}$ \\
\hline 15 & 0.34 & 1.94 & $8.906 \times 10^{-11}$ & $1.067 \times 10^{-9}$ & $2.718 \times 10^{-11}$ & $6.747 \times 10^{-10}$ & $1.529 \times 10^{-9}$ & $2.053 \times 10^{-9}$ \\
\hline 15 & 3.43 & 1.51 & $8.087 \times 10^{-11}$ & $1.551 \times 10^{-9}$ & $3.000 \times 10^{-10}$ & $5.965 \times 10^{-10}$ & $1.003 \times 10^{-9}$ & $1.779 \times 10^{-9}$ \\
\hline 15 & 2.06 & 1.59 & $8.255 \times 10^{-11}$ & $1.175 \times 10^{-9}$ & $1.263 \times 10^{-10}$ & $4.812 \times 10^{-10}$ & $1.198 \times 10^{-9}$ & $1.780 \times 10^{-9}$ \\
\hline 15 & 2.24 & 1.56 & $8.171 \times 10^{-11}$ & $1.166 \times 10^{-9}$ & $1.120 \times 10^{-10}$ & $5.117 \times 10^{-10}$ & $1.161 \times 10^{-9}$ & $1.766 \times 10^{-9}$ \\
\hline 15 & 2.60 & 1.52 & $7.978 \times 10^{-11}$ & $1.180 \times 10^{-9}$ & $1.756 \times 10^{-10}$ & $5.697 \times 10^{-10}$ & $1.106 \times 10^{-9}$ & $1.747 \times 10^{-9}$ \\
\hline 15 & 0.74 & 1.73 & $8.497 \times 10^{-11}$ & $1.401 \times 10^{-9}$ & $1.573 \times 10^{-10}$ & $4.986 \times 10^{-10}$ & $1.312 \times 10^{-9}$ & $1.960 \times 10^{-9}$ \\
\hline 15 & 0.82 & 1.88 & $9.004 \times 10^{-11}$ & $1.131 \times 10^{-9}$ & $9.491 \times 10^{-11}$ & $6.329 \times 10^{-10}$ & $1.449 \times 10^{-9}$ & $1.945 \times 10^{-9}$ \\
\hline 15 & 10.7 & 1.53 & $9.683 \times 10^{-11}$ & $1.437 \times 10^{-9}$ & $1.429 \times 10^{-10}$ & $4.469 \times 10^{-10}$ & $1.176 \times 10^{-9}$ & $1.675 \times 10^{-9}$ \\
\hline 15 & 2.47 & 1.52 & $9.512 \times 10^{-11}$ & $1.354 \times 10^{-9}$ & $1.145 \times 10^{-10}$ & $4.935 \times 10^{-10}$ & $1.145 \times 10^{-9}$ & $1.733 \times 10^{-9}$ \\
\hline 15 & 4.79 & 1.50 & $9.049 \times 10^{-11}$ & $2.026 \times 10^{-9}$ & $3.270 \times 10^{-10}$ & $5.840 \times 10^{-10}$ & $8.844 \times 10^{-10}$ & $1.721 \times 10^{-9}$ \\
\hline 15 & 2.63 & 1.53 & $9.902 \times 10^{-11}$ & $1.755 \times 10^{-9}$ & $2.502 \times 10^{-10}$ & $5.771 \times 10^{-10}$ & $1.016 \times 10^{-9}$ & $1.714 \times 10^{-9}$ \\
\hline 20 & 2.85 & 1.74 & $8.829 \times 10^{-12}$ & $4.731 \times 10^{-10}$ & $1.596 \times 10^{-10}$ & $2.043 \times 10^{-9}$ & $6.253 \times 10^{-9}$ & $7.217 \times 10^{-9}$ \\
\hline 20 & 1.47 & 2.23 & $3.026 \times 10^{-13}$ & $3.264 \times 10^{-10}$ & $1.510 \times 10^{-10}$ & $2.042 \times 10^{-9}$ & $6.133 \times 10^{-9}$ & $7.058 \times 10^{-9}$ \\
\hline 20 & 2.50 & 1.93 & $6.432 \times 10^{-12}$ & $4.977 \times 10^{-10}$ & $1.442 \times 10^{-10}$ & $2.069 \times 10^{-9}$ & $6.196 \times 10^{-9}$ & $7.106 \times 10^{-9}$ \\
\hline 20 & 5.03 & 1.74 & $5.086 \times 10^{-11}$ & $9.943 \times 10^{-10}$ & $4.121 \times 10^{-10}$ & $1.749 \times 10^{-9}$ & $5.808 \times 10^{-9}$ & $6.879 \times 10^{-9}$ \\
\hline 20 & 2.76 & 1.76 & $9.208 \times 10^{-12}$ & $4.776 \times 10^{-10}$ & $1.581 \times 10^{-10}$ & $2.039 \times 10^{-9}$ & $6.232 \times 10^{-9}$ & $7.192 \times 10^{-9}$ \\
\hline 20 & 2.60 & 1.90 & $1.050 \times 10^{-11}$ & $4.893 \times 10^{-10}$ & $1.569 \times 10^{-10}$ & $2.036 \times 10^{-9}$ & $6.206 \times 10^{-9}$ & $7.159 \times 10^{-9}$ \\
\hline 20 & 2.43 & 1.86 & $8.884 \times 10^{-12}$ & $4.709 \times 10^{-10}$ & $1.569 \times 10^{-10}$ & $2.047 \times 10^{-9}$ & $6.254 \times 10^{-9}$ & $7.229 \times 10^{-9}$ \\
\hline 20 & 1.04 & 2.47 & $1.096 \times 10^{-13}$ & $3.264 \times 10^{-10}$ & $1.337 \times 10^{-10}$ & $1.888 \times 10^{-9}$ & $5.530 \times 10^{-9}$ & $6.396 \times 10^{-9}$ \\
\hline 20 & 4.15 & 1.85 & $5.069 \times 10^{-11}$ & $9.497 \times 10^{-10}$ & $3.058 \times 10^{-10}$ & $1.884 \times 10^{-9}$ & $5.892 \times 10^{-9}$ & $6.981 \times 10^{-9}$ \\
\hline 20 & 0.65 & 3.03 & $9.568 \times 10^{-14}$ & $3.264 \times 10^{-10}$ & $8.813 \times 10^{-11}$ & $1.576 \times 10^{-9}$ & $4.121 \times 10^{-9}$ & $4.824 \times 10^{-9}$ \\
\hline 20 & 0.78 & 2.85 & $1.001 \times 10^{-13}$ & $3.264 \times 10^{-10}$ & $1.027 \times 10^{-10}$ & 1. $676 \times 10^{-9}$ & $4.574 \times 10^{-9}$ & $5.329 \times 10^{-9}$ \\
\hline 20 & 0.84 & 2.62 & $1.056 \times 10^{-13}$ & $3.264 \times 10^{-10}$ & $1.214 \times 10^{-10}$ & $1.804 \times 10^{-9}$ & $5.150 \times 10^{-9}$ & $5.972 \times 10^{-9}$ \\
\hline 20 & 1.00 & 2.35 & $1.123 \times 10^{-13}$ & $3.264 \times 10^{-10}$ & $1.434 \times 10^{-10}$ & $1.955 \times 10^{-9}$ & $5.832 \times 10^{-9}$ & $6.733 \times 10^{-9}$ \\
\hline 20 & 1.65 & 1.78 & $5.378 \times 10^{-12}$ & $4.466 \times 10^{-10}$ & $1.577 \times 10^{-40}$ & $2.066 \times 10^{-9}$ & $6.304 \times 10^{-9}$ & $7.300 \times 10^{-9}$ \\
\hline 20 & 0.75 & 2.76 & $1.022 \times 10^{-13}$ & $3.264 \times 10^{-10}$ & $1.101 \times 10^{-10}$ & $1.726 \times 10^{-9}$ & $4.800 \times 10^{-9}$ & $5.581 \times 10^{-9}$ \\
\hline 20 & 0.53 & 3.40 & $1.054 \times 10^{-13}$ & $3.273 \times 10^{-10}$ & $5.806 \times 10^{-11}$ & $1.369 \times 10^{-9}$ & $3.191 \times 10^{-9}$ & $3.784 \times 10^{-9}$ \\
\hline 20 & 4.33 & 1.87 & $7.359 \times 10^{-11}$ & $1.499 \times 10^{-9}$ & 3. $478 \times 10^{-10}$ & $1.823 \times 10^{-9}$ & $5.809 \times 10^{-9}$ & $6.897 \times 10^{-9}$ \\
\hline 20 & 0.81 & 2.70 & $1.037 \times 10^{-13}$ & $3.264 \times 10^{-10}$ & $1.150 \times 10^{-10}$ & $1.761 \times 10^{-9}$ & $4.954 \times 10^{-9}$ & $5.753 \times 10^{-9}$ \\
\hline 20 & 1.19 & 2.28 & $1.148 \times 10^{-13}$ & $3.264 \times 10^{-10}$ & $1.492 \times 10^{-10}$ & $1.995 \times 10^{-9}$ & $6.008 \times 10^{-9}$ & $6.929 \times 10^{-9}$ \\
\hline 20 & 1.52 & 1.97 & $5.638 \times 10^{-12}$ & $4.365 \times 10^{-10}$ & $1.577 \times 10^{-10}$ & $2.065 \times 10^{-9}$ & $6.301 \times 10^{-9}$ & $7.301 \times 10^{-9}$ \\
\hline 20 & 1.39 & 1.93 & $5.328 \times 10^{-12}$ & $4.409 \times 10^{-10}$ & $1.577 \times 10^{-10}$ & $2.067 \times 10^{-9}$ & $6.305 \times 10^{-9}$ & $7.303 \times 10^{-9}$ \\
\hline 20 & 8.86 & 1.74 & $8.004 \times 10^{-11}$ & $2.496 \times 10^{-9}$ & $4.176 \times 10^{-10}$ & $1.397 \times 10^{-9}$ & $4.789 \times 10^{-9}$ & $6.023 \times 10^{-9}$ \\
\hline 20 & 0.85 & 2.62 & $1.057 \times 10^{-13}$ & $3.264 \times 10^{-10}$ & $1.215 \times 10^{-10}$ & $1.805 \times 10^{-9}$ & $5.152 \times 10^{-9}$ & $5.975 \times 10^{-9}$ \\
\hline 25 & 1.92 & 3.13 & $2.850 \times 10^{-10}$ & $1.230 \times 10^{-8}$ & $3.117 \times 10^{-10}$ & $1.711 \times 10^{-9}$ & $6.031 \times 10^{-9}$ & $9.070 \times 10^{-9}$ \\
\hline 25 & 3.07 & 1.83 & $2.844 \times 10^{-10}$ & $1,230 \times 10^{-8}$ & $3.117 \times 10^{-10}$ & $1.711 \times 10^{-9}$ & $6.031 \times 10^{-9}$ & $9.070 \times 10^{-9}$ \\
\hline 25 & 3.30 & 2.35 & $3.073 \times 10^{-10}$ & $1.212 \times 10^{-8}$ & $3.031 \times 10^{-10}$ & $1.735 \times 10^{-9}$ & $6.110 \times 10^{-9}$ & $9.142 \times 10^{-9}$ \\
\hline 25 & 1.04 & 1.84 & $1.789 \times 10^{-10}$ & $5.907 \times 10^{-9}$ & $3.810 \times 10^{-10}$ & $2.215 \times 10^{-9}$ & $7.333 \times 10^{-9}$ & $9.925 \times 10^{-9}$ \\
\hline 25 & 9.73 & 2.35 & $2.605 \times 10^{-10}$ & $1.109 \times 10^{-8}$ & $2.730 \times 10^{-10}$ & $1.597 \times 10^{-9}$ & $5.577 \times 10^{-9}$ & $8.421 \times 10^{-9}$ \\
\hline 25 & 1.20 & 1.84 & $1.789 \times 10^{-10}$ & $5.907 \times 10^{-9}$ & $3.810 \times 10^{-10}$ & $2.215 \times 10^{-9}$ & $7.333 \times 10^{-9}$ & $9.925 \times 10^{-9}$ \\
\hline 25 & 7.42 & 2.37 & $1.401 \times 10^{-10}$ & $4.068 \times 10^{-9}$ & $3.886 \times 10^{-10}$ & $1.576 \times 10^{-9}$ & $5.379 \times 10^{-9}$ & $7.444 \times 10^{-9}$ \\
\hline 25 & 18.4 & 2.35 & $2.645 \times 10^{-10}$ & $1.142 \times 10^{-8}$ & $4.609 \times 10^{-10}$ & $1.625 \times 10^{-9}$ & $5.474 \times 10^{-9}$ & $8.339 \times 10^{-9}$ \\
\hline 25 & 14.8 & 2.35 & $5.072 \times 10^{-10}$ & $2.205 \times 10^{-8}$ & $4.837 \times 10^{-10}$ & $1.006 \times 10^{-9}$ & $3.374 \times 10^{-9}$ & $6.887 \times 10^{-9}$ \\
\hline 25 & 2.78 & 2.35 & $3.069 \times 10^{-10}$ & $1.212 \times 10^{-8}$ & $3.075 \times 10^{-10}$ & $1.737 \times 10^{-9}$ & $6.110 \times 10^{-9}$ & $9.142 \times 10^{-9}$ \\
\hline 25 & 0.89 & 4.66 & $1.248 \times 10^{-13}$ & $1.743 \times 10^{-10}$ & $3.497 \times 10^{-10}$ & $1.585 \times 10^{-9}$ & $4.685 \times 10^{-9}$ & $5.718 \times 10^{-9}$ \\
\hline 25 & & 2.35 & $3.073 \times 10^{-10}$ & $1.212 \times 10^{-8}$ & $2.926 \times 10^{-10}$ & $1.730 \times 10^{-9}$ & $6.110 \times 10^{-9}$ & $9.142 \times 10^{-9}$ \\
\hline 25 & 0.92 & 1.84 & $1.789 \times 10^{-10}$ & $5.907 \times 10^{-9}$ & $3.810 \times 10^{-10}$ & $2.215 \times 10^{-9}$ & $7.333 \times 10^{-9}$ & $9.925 \times 10^{-9}$ \\
\hline 25 & & 2.35 & $3.069 \times 10^{-10}$ & $1.212 \times 10^{-8}$ & $3.094 \times 10^{-10}$ & $1.738 \times 10^{-9}$ & $6.110 \times 10^{-9}$ & $9.142 \times 10^{-9}$ \\
\hline & 0 & 2.38 & $1.350 \times 10^{-10}$ & $3.942 \times 10^{-9}$ & $3.500 \times 10^{-10}$ & $1.592 \times 10^{-9}$ & $5.492 \times 10^{-9}$ & $7.463 \times 10^{-9}$ \\
\hline & & 2.38 & $1.222 \times 10^{-10}$ & $3.644 \times 10^{-9}$ & $3.403 \times 10^{-10}$ & $1.988 \times 10^{-9}$ & $6.676 \times 10^{-9}$ & $8.481 \times 10^{-9}$ \\
\hline & 1.52 & 1.83 & $1.789 \times 10^{-10}$ & $5.907 \times 10^{-9}$ & $3.810 \times 10^{-10}$ & $2.215 \times 10^{-9}$ & $7.333 \times 10^{-9}$ & $9.925 \times 10^{-9}$ \\
\hline & 1.57 & 3.73 & $5.140 \times 10^{-12}$ & $1.697 \times 10^{-10}$ & $3.720 \times 10^{-10}$ & $2.058 \times 10^{-9}$ & $6.608 \times 10^{-9}$ & $7.903 \times 10^{-9}$ \\
\hline 25 & 0.99 & 4.89 & $1.422 \times 10^{-13}$ & $1.740 \times 10^{-10}$ & $3.463 \times 10^{-10}$ & $1.418 \times 10^{-9}$ & $4.150 \times 10^{-9}$ & $5.074 \times 10^{-9}$ \\
\hline 40 & 8.40 & 2.38 & $2.614 \times 10^{-10}$ & $1.109 \times 10^{-8}$ & $3.464 \times 10^{-10}$ & $1.625 \times 10^{-9}$ & $5.574 \times 10^{-9}$ & $8.420 \times 10^{-9}$ \\
\hline 25 & 2.53 & 2.35 & $3.069 \times 10^{-10}$ & $1.212 \times 10^{-8}$ & $3.113 \times 10^{-10}$ & $1.739 \times 10^{-9}$ & $6.110 \times 10^{-9}$ & $9.142 \times 10^{-9}$ \\
\hline 25 & 4.72 & 2.35 & $3.077 \times 10^{-10}$ & $1.212 \times 10^{-8}$ & $2.952 \times 10^{-10}$ & $1.731 \times 10^{-9}$ & $6.110 \times 10^{-9}$ & $9.143 \times 10^{-9}$ \\
\hline
\end{tabular}

Table A5. Complete table of decayed isotopic yields for all models in mass fraction. $\mathrm{M}_{\text {prog }}$ is the progenitor mass, $\mathrm{E}_{\exp }$ is the explosion energy, $\mathrm{M}_{\text {rem }}$ is the remnant mass. 